\title{
Orthoptera Tettigoniidae as indicators of biodiversity hotspots in the Guinean Forests of Central and West Tropical Africa
}

\author{
BRUNO MASSA ${ }^{1}$ \\ ${ }^{1}$ Department of Agriculture, Food and Forest Sciences, University of Palermo, Viale Scienze 13, 90128 Palermo, Italy (retired). \\ झ"bruno.massa@unipa.it; @ https://orcid.org/0000-0003-2127-0715
}

\begin{abstract}
The present paper has two aims: 1) to present the results of the study of selected species of Orthoptera Tettigoniidae collected in the Guinean forests of West Africa and in the important hotspot of Dzanga-Ndoki National Park (Central African Republic); 2) to carry out a check-list of six subfamilies of Tettigoniidae (Pseudophyllinae, Conocephalinae, Hexacentrinae, Phaneropterinae, Mecopodinae and Hetrodinae) living in Central-West tropical Africa, in particular in two main tropical forests, in the subregion of upper Guinea, and in the subregion Nigeria-Cameroon plus the biodiversity hotspots of Central African Republic and Gabon. Many new records are reported and the following new species are described: Plangia astylata n. sp. from Central African Republic and Gabon, Plangia chopardi n. sp. from Côte d'Ivoire, and Catoptropteryx lineata n. sp. from Liberia. In addition the male of Plangia karschi Chopard, 1954 is described and some taxonomical notes on the recently described Arantia marginata Massa, 2021 are discussed. The new name Pseudorhynchus raggei is proposed for Pseudorhynchus robustus Ragge, 1969, junior primary homonym of Pseudorhynchus robustus Willemse, 1953.

Then, the author lists all the Tettigoniidae of the above listed subfamilies presently known in central-west tropical Africa (Guinean forests). This wide tropical area holds important biodiversity hotspots that the author highlights through the study of katydids. Many groups of species tend to isolate and speciate probably more than other groups of insects. Overall, the total number amounts to 332 species, of which 242 live in Cameroon-Nigeria subregion plus Central African Republic and Gabon, 216 in the Upper Guinea subregion. The occurrence of endemic taxa is 35.9 and $40.3 \%$, respectively; this resulted a very high percentage compared to that known for plants and animals in the area. Starting from the list of Tettigoniidae three remarkable biodiversity hotspots were examined, Dzanga-Ndoki National Park (Central African Republic), Mt. Tonkoui and Taï National Park (both in Côte d'Ivoire); overall they hold 134, 81 and 88 species, respectively. It seems that in tropical Africa there is a specific richness gradient with an East-West impoverishment, but also a possible increase of endemism occurrence. However, presently this richness suffers a high decline risk, due to deforestation and environmental degradation, in turn dependent on the inequality between human populations, wars and political instability in some tropical areas.
\end{abstract}

Key words: check-list, new species, taxonomy, new records, distribution, endemic taxa, forest conservation

\section{Introduction}

The Guinean Forests of West Africa are critically endangered, and only few remains of old primary forests survive. Main tropical Guinean forests lie in the countries of Liberia, Côte d'Ivoire, Ghana, Togo, Benin, Nigeria, Equatorial Guinea and São Tomé and Principe, and eastwards to Sanaga River in Cameroon. Generally two subregions are recognized: 1) upper Guinea from Guinea to east Sierra Leone and through Liberia, Côte d'Ivoire and Ghana to Togo; 2) the Nigeria-Cameroon extending along the coast from west Nigeria to Sanaga river in Cameroon. The two subregions are separated by a wide area, that presently is a mixture of farmland, derived savanna woodland, and relict patches of dry forests (Bakarr et al. 2004, Naskrecki 2008). Further, forests of Gabon and the southern part of Central African Republic show a certain continuity with the West African Guinean forests (see Massa 2021a). Of the original extent of Guinean forest of $620,314 \mathrm{~km}^{2}$ only 93,047 (15\%) remain (Mittemeier et al. 2004). In many of these forests some biodiversity hotspots have been recognized, mainly based on botanical aspects, but later also on zoological distinctive peculiarities, like the species richness and the number of endemic taxa. The Guinean Forest 
hotspots contain a rich and unique faunal assemblage, many species are endemic and tend to have highly restricted ranges within the forest. Biodiversity hotspots (covering just $16 \%$ of the Earth's land) contain about $32 \%$ of all humans. In particular Guinean Forests of West Africa inhabit a human population density of $144 \mathrm{~km}^{-2}$, with a child malnutrition rate of $21 \%$ (Mittelmeier et al. 2011). Presently, the major threat is deforestation, due to commercial logging and slash-and-burn agriculture, both of which are prevalent in all Guinean forest countries (Mittelmeier et al. 2011).

Since the late 1960s efforts have been under way in all the countries to establish more strictly controlled and effectively managed protected areas, but the area of forest strictly protected is still small compared to the forest area remaining in each country. To ensure survival of many forest species, it is imperative that remaining forest areas be given more adequate protection and that as many existing forest reserves as possible be elevated to National Park status (Bakarr et al. 2004, Mittermeier et al. 2011), but it is also imperative to assure the human populations a better standard of living than the present one, and this is possible only with the intervention of richer countries. Sustainability can only be achieved through human actions based on the qualities of our existence, capable of fully respecting the biophysical limits and dynamic balances of the Earth.

Titley et al. (2017) have noticed a considerable taxonomic weighting towards vertebrates and an under-representation of invertebrates in the published literature. They observed that this discrepancy is more pronounced in highly cited papers, and in tropical regions, with only $43 \%$ of biodiversity research in the tropics including invertebrates; overall, tropical countries were understudied relative to temperate countries. Thus, biodiversity studies focusing on invertebrates are certainly welcome.

The number of species alone is an inappropiate index of conservation priority, while high levels of endemism occurrence are certainly more informative. We must conserve the areas with high levels of endemism because they contain species that cannot be saved elsewhere (Mittermeier et al. 2011). These areas are among the most important hotspots of biodiversity. Nevertheless, much of this wide African tropical region is little explored from the entomological point of view, and we know that insects are the most abundant species living on the Earth. Thus, any information on the species richness and the endemism occurrence of selected insect groups will certainly be used for conservation purposes.

The African Natural History Research Trust (ANHRT, Hereford, UK) is a Research Institute dedicated to the study of African insects, primarily Lepidoptera. ANHRT organizes entomological missions to promote collectionsbased faunistic and taxonomic research. The team of ANHRT organizes and conducts collecting and research expeditions to a wide range of countries in Africa, in partnership with host institutions and government bodies; possibly the same collecting sites are visited over a number of years and in different seasons to build up a picture of the insect fauna at each site.

The ANHRT sent on loan to me 2250 specimens of Orthoptera (mainly Phaneropterinae, Conocephalinae and Pseudophyllinae) collected during the entomological expeditions to Senegal, Sierra Leone, Liberia, Guinea, Togo, Côte d'Ivoire and Principe Is. to be identified. Further, I personally took part to an expedition to Côte d'Ivoire (Taï National Park) organized by Philippe Moretto (Association Catharsius) in 2017, who also sent me some other specimens collected in other Ivorian localities, in Senegal, in Burkina-Faso and in the area of the Dzanga-Ndoki National Park and Dzanga-Sangha Special Reserve in Central African Republic (see Massa et al. 2020). A small lot of specimens collected in Ghana in 1982-1985 by the Italian entomologist Pietro Butti was presented to me by Vincenzo Vomero. Overall, more than 7000 specimens were examined. The study of this high number of specimens allowed to have a fairly clear idea of the species richness and the endemism occurrence.

The present paper has two aims: 1) to present the results of the study of selected species of Orthoptera Tettigoniidae collected in the Guinean forests of West Africa and in the important hotspot of Dzanga-Ndoki National Park (Central African Republic); 2) to try to carry out a check-list of six subfamilies of Tettigoniidae (Pseudophyllinae, Conocephalinae, Hexacentrinae, Phaneropterinae, Mecopodinae and Hetrodinae) living in Central-West tropical Africa, in particular in two main tropical forests, in the subregion of upper Guinea, and in the subregion NigeriaCameroon plus the biodiversity hotspots of Central African Republic and Gabon. This check-list represents the basis from which to extrapolate two important information, like the number of species and the endemism occurrence.

\section{Material and Methods}

During the collecting sessions sampling was always carried out both during daytime and nighttime. Various stan- 
dard techniques, such as visual search, sweeping, beating, etc. were used at all times; sampling method of staff of ANHRT was focused on Lepidoptera, but other insect orders were also sampled. Two main methods to collect Orthoptera were used: 1) Hand collecting of grasshoppers and katydids using a beating tray or sweep net, or simply by searching vegetation. 2) Light trap: many Ensifera are attracted to a specially engineered white tent containing a bright mercury vapour bulb where they can be collected. The ANHRT-designed Edward's Trap is placed under the bulb collecting the smallest insects which would otherwise be missed. As different insects are attracted to different wavelengths of light, smaller ultraviolet lights are also used, sometimes in combination with an automatic bucket trap. The most fruitful method to collect Orthoptera is the Light trap, that has been used with different kinds of lamps, which revealed to be very attractive for most Ensifera species. Lamps were of four types: ultraviolet lights (UV), bright mercury vapour bulb, 300-700nm (LepiLED trap), actinic light, 350-400nm (Actinic trap), and mercury vapour light (MV trap).

Some specimens were photographed with a Nikon Coolpix 4500 digital camera, mounted on a Wild M5 or Exacta Optech SL Stereomicroscopes and photographs were integrated using the freeware CombineZP (Hadley 2008) and processed in Adobe Photoshop CS4; further some specimens were photographed with an Olympus Stylus TG-5 Tough (cf. Mertens et al. 2017). Mounted specimens were measured with a digital caliper (precision $0.01 \mathrm{~mm}$ ); the following measurements were taken (in mm): Body length: dorsal length from the head to the apex of the abdomen; Pronotum length and height; Tegmina: length and maximum width of tegmina; Hind femora length; Ovipositor: maximum length, subgenital plate included. The shape of the stridulatory file under the male's left forewing and the number and arrangement of the teeth are useful characters that identify whether a species is bioacoustically separated from another one (Ragge 1980, Heller 2006). Some identifications and comparisons were possible by consulting the web-site Orthoptera Species File (http://orthoptera.speciesfile.org/HomePage/Orthoptera/HomePage.aspx), where many photos are available.

The final list of species includes Tettigoniidae of the subfamilies Pseudophyllinae, Conocephalinae, Hexacentrinae, Phaneropterinae, Mecopodinae and Hetrodinae. The list has been obtained after the consultation of Orthoptera Species File online (Cigliano et al. 2021), the ANHRT and the personal collections. The list includes also seven undescribed species of the genus Eurycorypha Stål, 1873, that will be described within a revision in collaboration with the colleague Claudia Hemp. Concerning the genus Lanista Bolívar, 1890 (Conocephalinae), following Naskrecki \& Guta (2019), the record of Lanista annulicornis (Walker, 1869) in the Dzanga-Ndoki National Park (Central African Republic) was changed into Lanista africana (Walker, 1871). Three hotspots, two in the Côte d'Ivoire (Taï Forest National Park and Mt. Tonkoui; cf. Moretto et al. 2021), one in the Central African Republic (Dzanga-Ndoki National Park, including also Dzanga-Sangha Special Reserve; cf. Massa et al. 2020) were explored extensively in different years and seasons; thus, in the list it is reported if each species has been detected in those hotspots.

Concerning the definition of endemism, it describes a taxon whose distribution is limited to a geographically confined territory, often small and localized. Thus, a taxon is endemic to a biogeographic region if it only occurs in that area (Massa \& Fontana 2020). In the present case, for the purposes of a valutation of the biodiversity hotspots, a species distributed in a wide area of Central and West Africa was not considered endemic, while a species living in a geographically confined territory (for example Upper Guinea in West Africa) was considered as endemic.

\section{Abbreviations used in the text}

ANHRT: African Natural History Research Trust, Hereford, UK

BMPC: Bruno Massa Personal Collection, Palermo, Italy

\section{Study areas}

Information on characteristics of the areas have been drawn from Guillaumet \& Adjanohoun (1970), White (1986), Olson et al. (2001), Moretto (2010), Atsri et al. (2018), Moretto et al. (2021) and communicated by P. Moretto and the ANHRT staff.

\section{Senegal (Figs. 1-2)}


ic, Lepiled Light Trap) 27.V-2.VI.2019 (West Sudanian savanna. Dry forest. Small relictual mesophiles riverine forests without any communication with any other hydrographic web); 2) Senegal, Simenti, Niokolo-Koba NP (30m) $13^{\circ} 1$ '33”N, 13¹7'4”W (MV Light Trap) 3-16.VI.2019 (Guinean forest-savanna mosaic. Dry forest, tree savanna, 
more or less mesophile riverine forests. Open savanna on bowés); 3) Senegal, Botou Village (50m) $13^{\circ} 48^{\prime} 28^{\prime \prime} \mathrm{N}$, 13³4'43”W (MV, Actinic, Lepiled Light Trap) 18-25.VI.2019 (West Sudanian savanna. Degraded dry forest). In all the sites collectors were: M. Aristophanous, P. Moretto, L. Mulvaney.

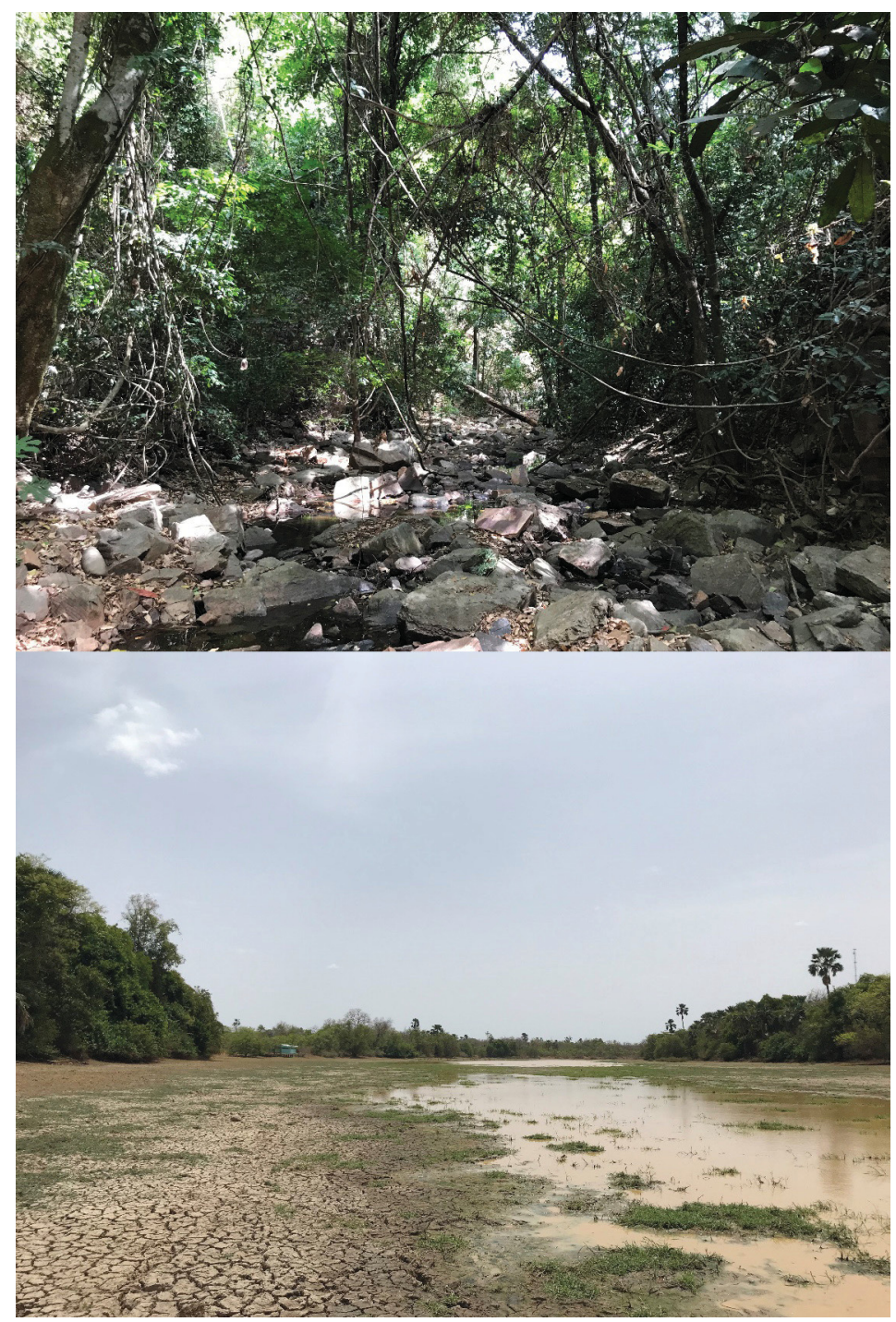

FIGS. 1-2. Senegal, Dindéfelo Community Nature Reserve, Gallery forest (above); Senegal, Mare de Simenti, Simenti Camp, Niokolo Koba National Park (bottom) (Photos by L. Mulvaney).

\section{Guinea (Figs. 3-7)}

The collecting sites were the following: 1) Guinea, Guinée forestière, Bossou For. \& Inst. Rech. Env. Bossou (690m) 7³8’32”N, 8³0’30”W (gen. coll., MV, Lepiled, 8w UV Cold Cathode Light Trap) 24-31.VI.2019, V. Dérozier, J. Suha Dore, S. Koivagui, W. Miles, S. Safian, R. Warner (Lowland rainforest and farmland close to village); 2) Guinea, Guinée forestière, Mt Nimba UNESCO World Heritage Site, Suengbara Village to Yie River Valley (700m) 7³6’56”N, 8²6’54’W (250W Blended Light Trap) 1-8.VII.2019, V. Dérozier, S. Koivagui, W. Miles, S. Safian, R. Warner (high altitude grassland with patches of gallery forest, Afromontane forest lower dow); 3) Guinea, Guinée forestière, Forêt Classée de Ziama, Sérédou (625m) 8²1 '26”N, 9¹7’48”W (Lepiled Light Trap) 916.VII.2019, V. Dérozier, S. Koivagui, W. Miles, S. Safian, R. Warner (lowland rainforest and farmland); 4) Guinea,

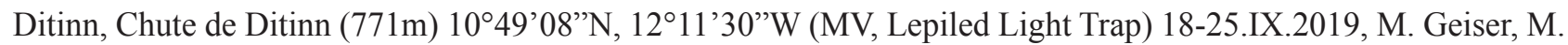
Leno, S. Koivagui, W. Miles, L. Mulvaney, S. Safian (Guinea savannah, woodland and gallery forest close to village); 5) Guinea, Dalaba, Forêt de Tinka (1289m) 1043'14”N, 12¹5'22”W (MV Light Trap) 25-28.IX.2019, M. Geiser, M. Leno, S. Koivagui, W. Miles, L. Mulvaney, S. Safian (degraded upland forest); 6) Guinea, Dalaba, Forêt de Goubel (1413m) 10³9’27'N, 12¹5’44”W (MV Light Trap) 10-18.IX.2019, M. Geiser, M. Leno, S. Koivagui, W. Miles, L. Mulvaney, S. Safian (Guinea savannah with patches of upland forest). 

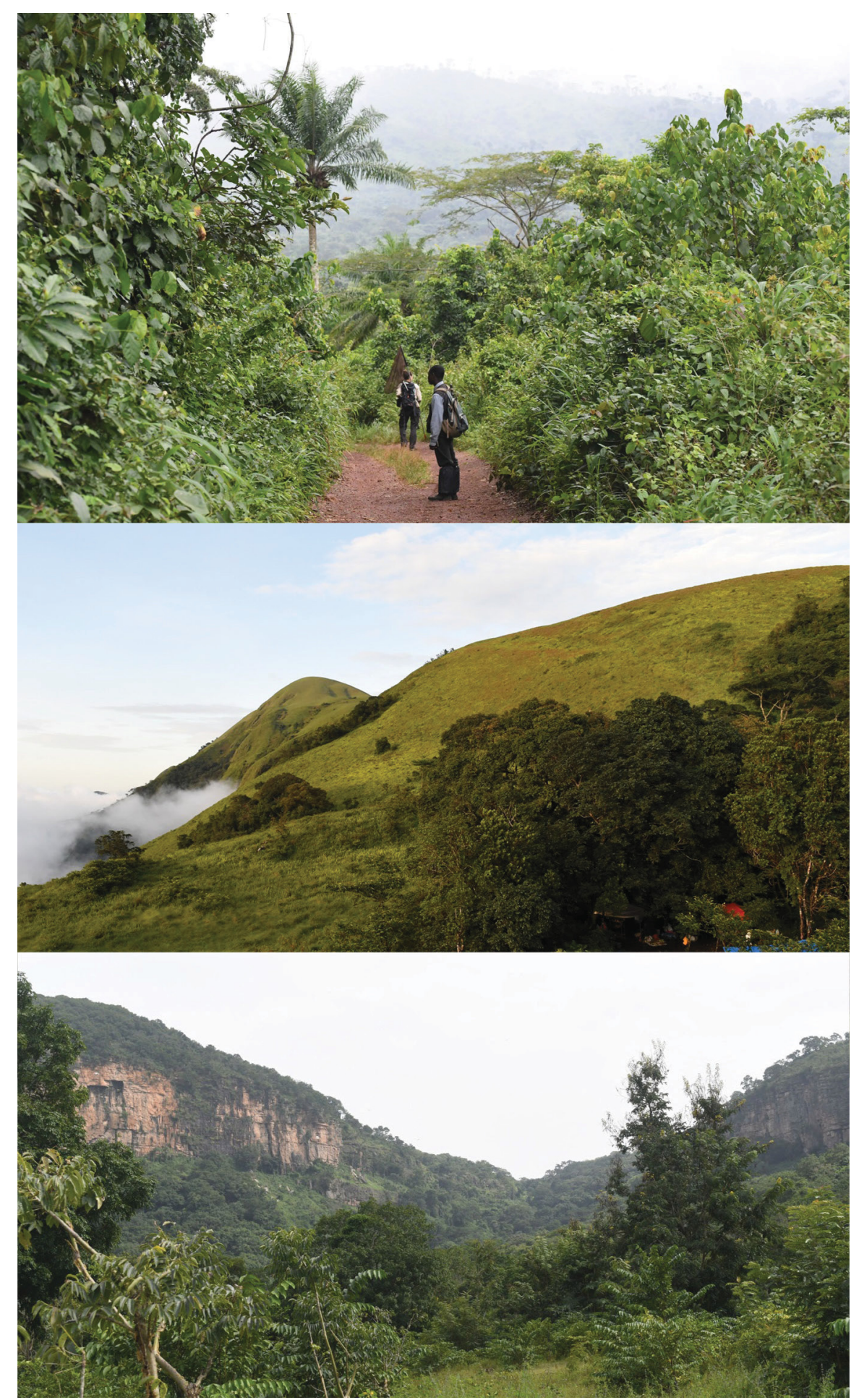

FIGS. 3-5. Guinea, Bossou—-track through forest (left) and farmland (right) (above); Guinea, Mt. Nimba—campsite in patch of gallery forest lower right, grassland and Mt. Richard Molard left (center); Guinea, Ditinn — edge of village, camp in woodland ahead, gallery forest in gorge top left (bottom) (Photos by W. Miles).

\section{Sierra Leone (Figs. 8-10)}

The collecting sites were the following: 1) Sierra Leone, Mansonia Village, foothills of Loma Mts. (420m) $9^{\circ} 07^{\prime} 47^{\prime} \mathrm{N}, 11^{\circ} 05^{\prime} 06^{\prime} \mathrm{W}$ (light trap) 6.VI.2016 (disturbed farmland/forest mosaic on the edge of the village); 2) Sierra Leone, Loma Mts., closed-canopy forest (1050m) 9 $9^{\circ} 10^{\prime} 35^{\prime \prime} \mathrm{N}, 11^{\circ} 05^{\prime} 25^{\prime} \mathrm{W}$ (light trap) 7-10.VI.2016 (pristine

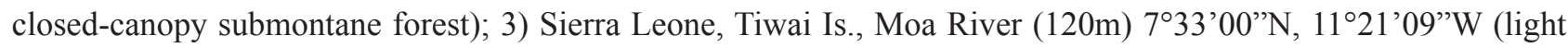
trap) 17-22.VI.2016 (small clearing in disturbed lowland rainforest near the river); 4) Sierra Leone, Kambama Vil- 


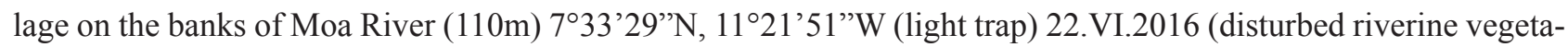
tion/farmland mosaic on the edge of the village). In all the sites collectors were: H. Takano, W. Miles, R. Goff.

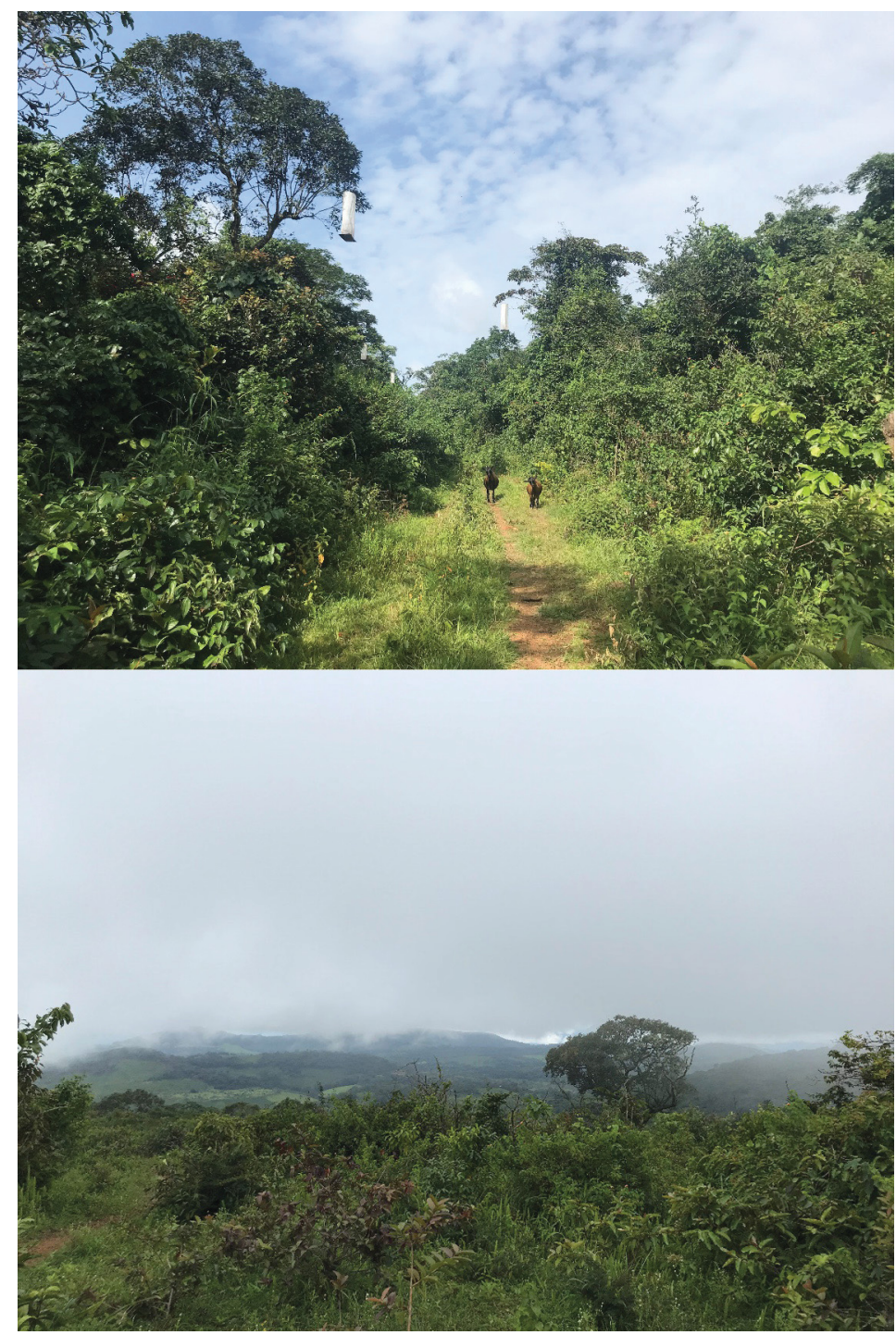

FIGS. 6-7. Guinea, Dalaba, Tinka forest (1289m) (above); Guinea, Dalaba, Goubel forest (1413m) (bottom) (Photos by L. Mulvaney).

\section{Liberia (Figs. 11-14)}

The collecting sites were the following: 1) Liberia, Lofa County, Zuwulor Vill. School (230m) 754'52"N, 9³1'08'W (Blended Bulb Trap) 8.XI.2017, M. Aristophanous, S. Safian, G. Simonics, L. Smith (village next to highly modified forest with plantations and farmland); 2) Liberia, Lofa County, Foya Proposed Protected Area (530m) 756’36”N, 10¹6’36”'W (MV, Cold Cathode UV, Actinic Light Trap) 10-19.XI.2017, M. Aristophanous, S. Safian, G. Simonics, L. Smith (wet lowland rainforest with closed canopy, some evidence of logging visible); 3) Liberia, Lofa County, Wologizi Mts., Base Camp Forest (611m) 807'17’N, 957’42”W (MV Light Trap) 20.XI1.XII.2017, M. Aristophanous, S. Safian, G. Simonics, L. Smith (disturbed lowland secondary rainforest, clear evidence of logging); 4) Liberia, Lofa County, Wologizi Mts., Ridge Camp 807’10”N, 9॰57’11”W (Cold Cathode Light Trap) 24-29.XI.2017, M. Aristophanous, S. Safian, G. Simonics, L. Smith (mid elevation wet rainforest. A big bush-fire (initiated by poachers) occurred here 2-3 years prior to the visit); 5) Liberia, Sinoe County, Krahn-Bassa Reserve, Juboe River, $7.5 \mathrm{~km}$ SW Pellokon town, 5’39'4"N, 8³9'4"W (general collection, MV, Cold Cathode UV Light Trap, Blended Bulb 250w Light Trap) 14-21.I.2018, M. Geiser, S. Safian, G. Simonics (lowland rainforest); 6) Liberia, Sinoe County, $6.5 \mathrm{~km}$ NW Jacksonville, Forest near Solve Problem Village (103m) 5'26’25'N, 97'39.9'W (MV Light Trap) 23-27.I.2018, M. Geiser, S. Safian, G. Simonics (disturbed lowland forest near village and road); 7) Liberia, Lofa County, Wologizi Mts., Rosewood Camp (585m) 806'14.09”N, 958'27.3”W 
(MV light trap) 18.XI-1.XII.2018, S. Safian G. Simonics (old-growth secondary lowland forest with patches of primary forest); 8) Liberia, Lofa County, Wologizi Mts., Ridge Camp 2 (883m) 87’20.79’'N, 956'50.75'W (Cold Cathode UV Trap), 22-31.XI.2018, S. Safian, G. Simonics (disturbed upland evergreen forest); 9) Liberia, Nimba County, Nimba Mts., ENNR Collcom Road (1000-1100m) 7³2’45.88’N, 8³1’21.04”W (Cold Cathode UV Trap) 16-28.XI.2018, S. Safian, G. Simonics (Upland wet rainforest with Parinari trees, and forest-savannah ecotone); 10) Liberia, Lofa County, Wologizi Mts., Elephant Ridge (1002m) 87'1.46”N, 955’24.18”W (Cold Cathode UV Trap) 23-26.XI.2018, S. Safian, G. Simonics (primary upland rainforest).

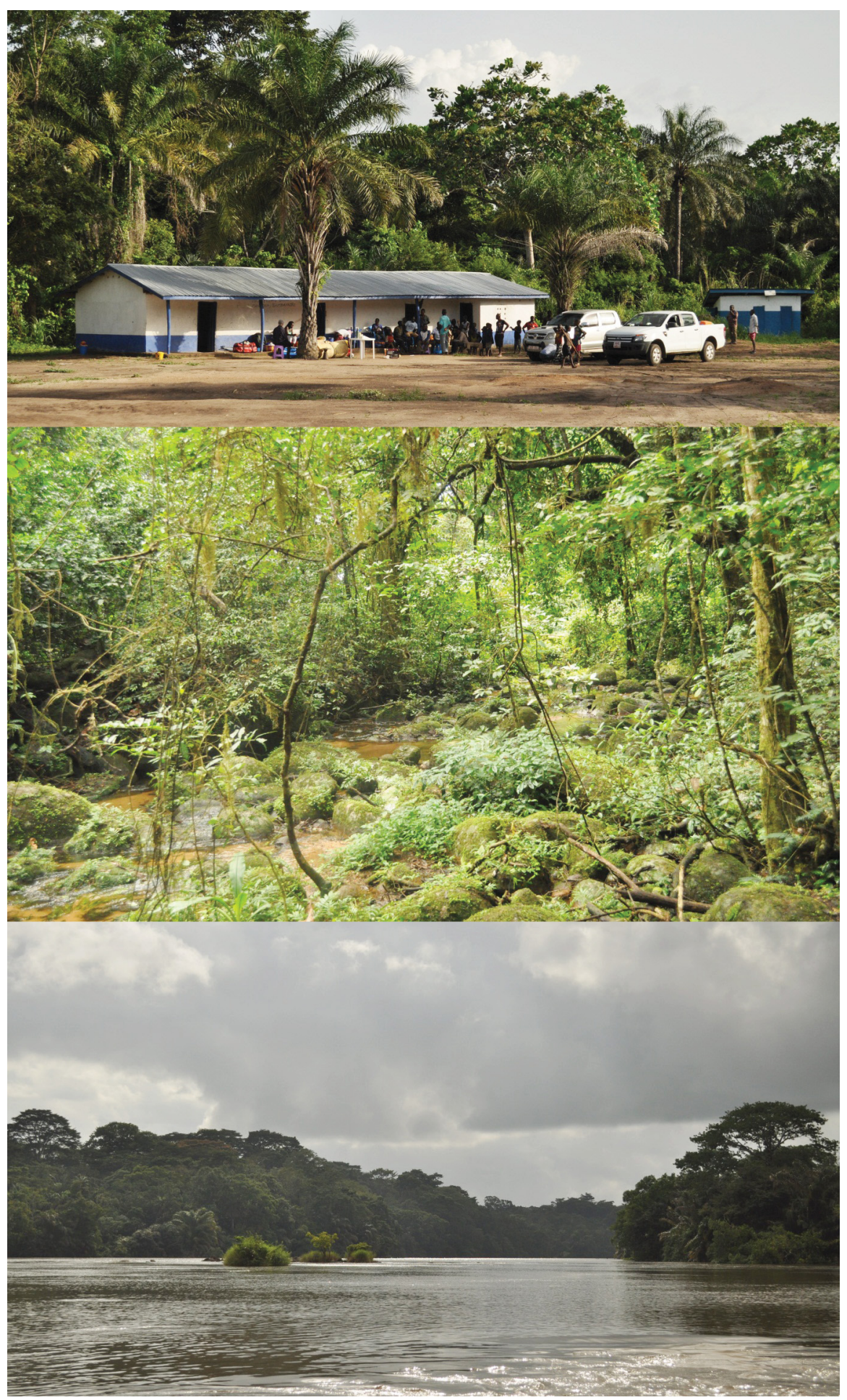

FIGS. 8-10. Sierra Leone, Mansonia Village, in the foothills of Loma Mts. (above); Sierra Leone, Loma Mts., closed-canopy forest (1050m) (center); Sierra Leone, Moa River and Tiwai Is. (bottom) (photos by W. Miles). 


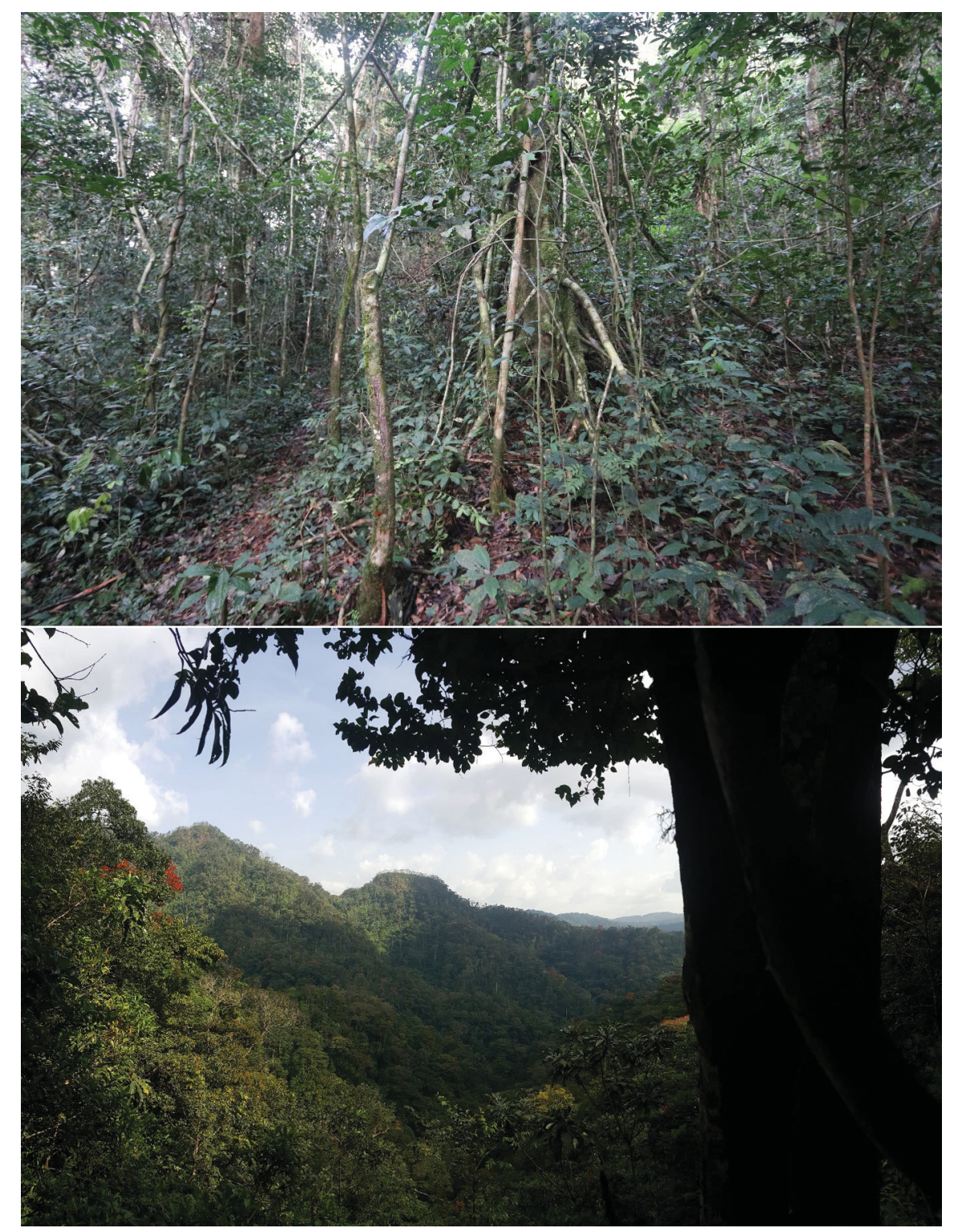

FIGS. 11-12. Liberia, Lofa County, Foya Proposed Protected Area (above); Liberia, Lofa County, Wologizi Mts, Ridge Camp (bottom) (photos by M. Aristophanous).

\section{Côte d'Ivoire (Figs. 15-23)}

The collecting sites were the following: 1) Côte d'Ivoire, Mt. Tonkoui Peak (1171m) $7^{\circ} 27^{\prime} 15.2^{\prime \prime} \mathrm{N}, 7^{\circ} 38^{\prime} 12.5^{\prime \prime} \mathrm{W}$ (light Trap) 9-16.IV.2016, 16-21.VII.2016, M. Aristophanous, P. Moretto; 2) Côte d'Ivoire, Mt. Tonkoui Peak 37.V.2017 (MV Light Trap), A. Aristophanous, M. Aristophanous, M. Geiser, P. Moretto; 3) Côte d'Ivoire, Mt. Tonkoui Peak 20-27.V.2018 (MV, Lepiled Light Trap), M. Aristophanous, W. Miles, P. Moretto, Y. Outtara (Mt. Tonkoui is covered by a typical Guinean montane forest, characterized by Parinari excelsa montane forest); 4) Côte d'Ivoire, Dolla Ranch (tree savannah) (481m) 758'7.7’N, 7³4’35.7'W (MV Light trap) 27.V-5.VI.2018, M. Aristophanous, W. Miles, P. Moretto, Y. Outtara (Guinean forest-savanna mosaic. Dry forest, degraded dry forest, mesophile spots and gallery forests); 5) Côte d'Ivoire, Abidjan, Banco Forest (Banco PN) (39-48m) 5'23’03.8’'N, 403'11.2’'W (MV Light Trap) 21-30.IV.2017, 21.IV-1.V.2017, A. Aristophanous, M. Aristophanous, M. Geiser, P. Moretto (eastern Guinean forest, humide psammohygrophile forest characterized by Turaeanthus africanus and Heisteria parvifolia. Primary forest and old secondary forest, characerized by Lophira alata); 6) Côte d'Ivoire, Taï National Park, Res. Station (174m) 5 50 5000”N, 7²0’32.0”'W (MV Light Trap) 25.III-17.IV.2017, A. Aristophanous, M. Aristophanous, M. Geiser, P. Moretto (western Guinean lowland forests. Primary and old secondary diversified wet forest. Gallery forests); 7) Côte d'Ivoire, Comoe, Kromambira Vill. (240m) 8³0’01.4’'N, 345'28.3”W (gen. coll. and light trap) 3-13.VIII.2016, M. Aristophanous, P. Moretto (Guinean forest-savanna mosaic. Dry forest, degraded dry for- 
est, mesophiles spots and gallery forests. Wooded savanna and open savanna); 8) Côte d'Ivoire, Yéalé Vill., Mt. Nimba, (380m) 7³1'35.3” N, 8²5’20.1'”W (gen. coll. and light trap) 18-29.IV.2016, 8.V.2016, M. Aristophanous, M. Geiser, P. Moretto (Western Guinean lowland forests. Degraded wet forest); 9) Côte d'Ivoire, Bossématié Classified Forest (180m) 6 $6^{\circ} 30^{\prime} 40^{\prime} \mathrm{N}, 3^{\circ} 28^{\prime} 35^{\prime \prime} \mathrm{W}$ (gen. coll. and light trap) 25-31.VII.2016, M. Aristophanous, P. Moretto (ecotone eastern Guinean forests/Guinean forest-savanna mosaic, degraded mesophile forest); 10) Côte d'Ivoire, Denguélé Classified Forest (sudanian forest) (479m) 9³0’0.6”N, 740’51.1”W (Lepiled Light Trap), 6-14.VI.2018, M. Aristophanous, W. Miles, P. Moretto, Y. Outtara; 11) Côte d'Ivoire, Denguélé Classified Forest (479m) (MV Light Trap, Lepiled Trap, Black Light Trap, gen. coll.) 11-18.XI.2019, M. Aristophanous, V. Dérozier, P. Moretto, S. Ouattara (West Sudanian savanna. Dry forest, clearings with Isoberlinia and Uapaca. Very similar to Fazao sites, Togo. Gallery forest and small areas of mesophile forest due to the presence of springs).

Additional samples were regularly collected at light by P. Moretto on Mt. Tonkoui in 2019 and 2020 in order to carry out a check-list of species living there and to show the high conservation value of this 'hotspot', and to propose the new Nature Reserve of Mt. Tonkoui (Moretto et al. 2021).
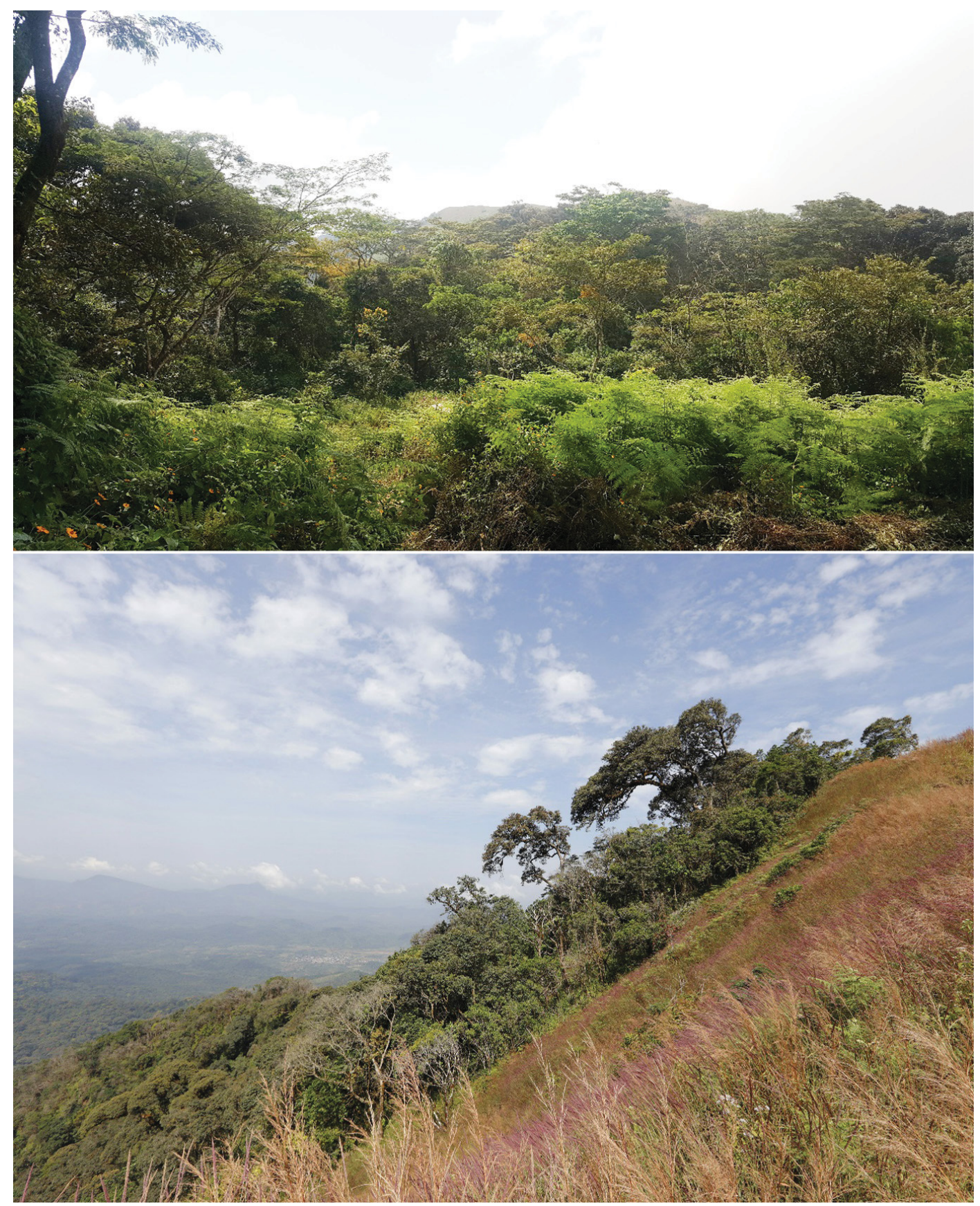

FIGS. 13-14. Liberia, Nimba County, Nimba Mts., ENNR Collcom Road (1000-1100m), upland wet rainforest with Parinari trees (above); Liberia, Mt. Nimba, forest-savannah ecotone (bottom) (photos by M. Aristophanous). 

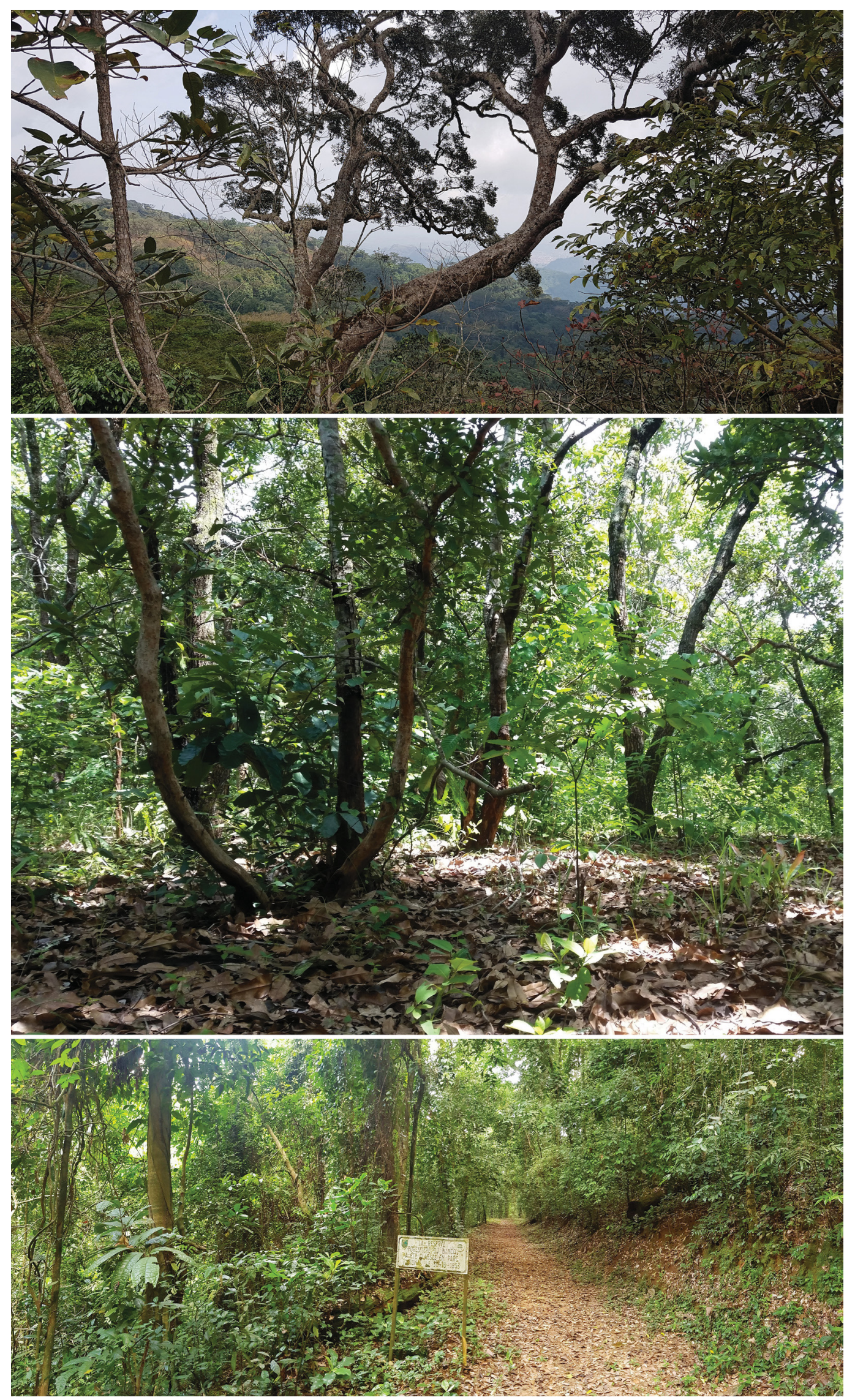

FIGS. 15-17. Côte d'Ivoire, Mt. Tonkoui Peak (1171m) (above); Côte d'Ivoire, Dolla Ranch (481m), Guinean degraded dry forest (center); Côte d'Ivoire, Abidjan, Banco Forest, Guinean humide psammohygrophile forest characterized by Turaeanthus africanus and Heisteria parvifolia (bottom) (photos by P. Moretto). 

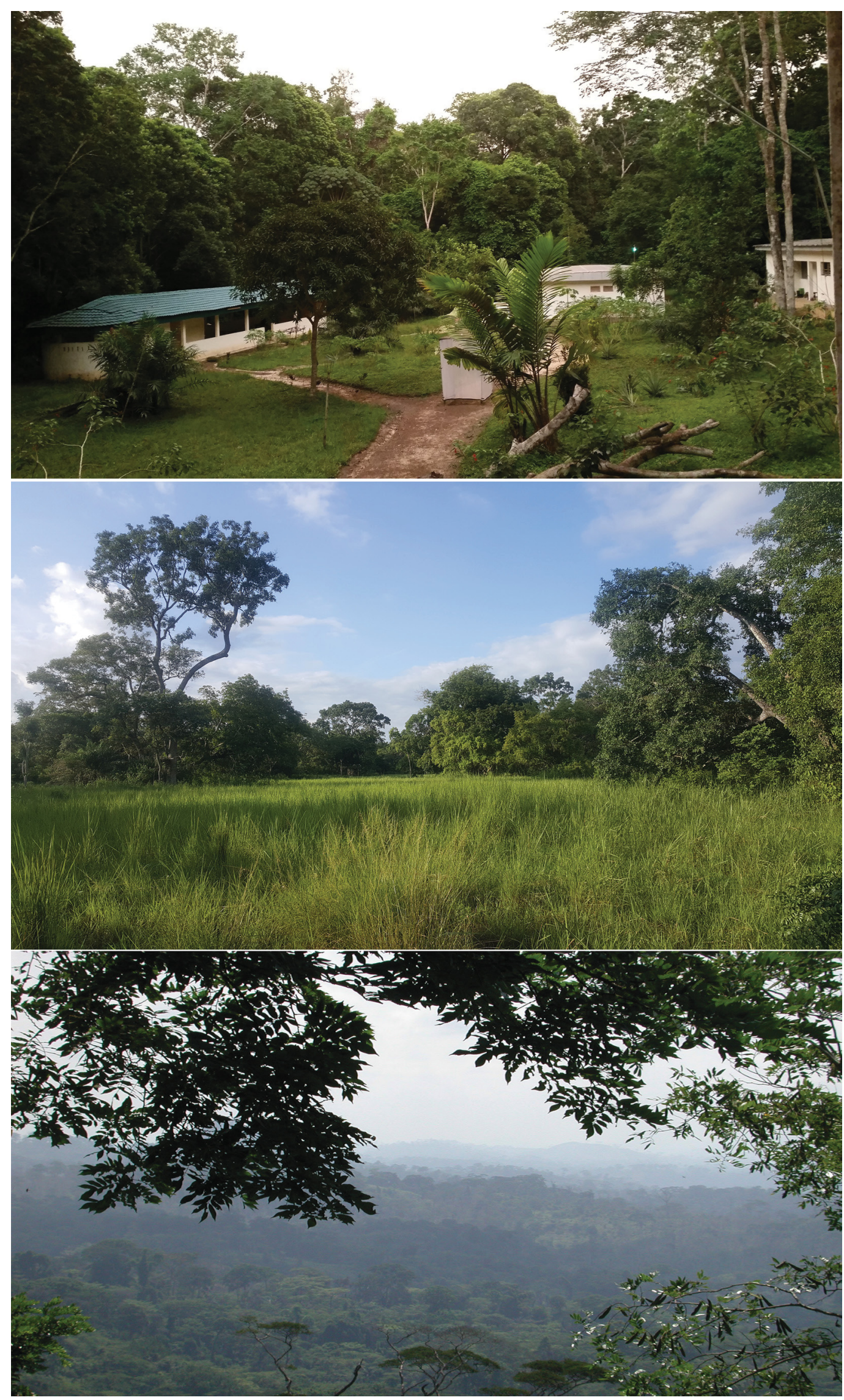

FIGS. 18-20. Côte d'Ivoire, Taï National Park, Research Station, Guinean lowland forest (above); Côte d'Ivoire, Comoe, Kromambira, mesophiles spots and gallery forest (center); Côte d'Ivoire, Yéalé, Mt. Nimba, (380m), Guinean lowland forest (bottom) (photos by P. Moretto). 

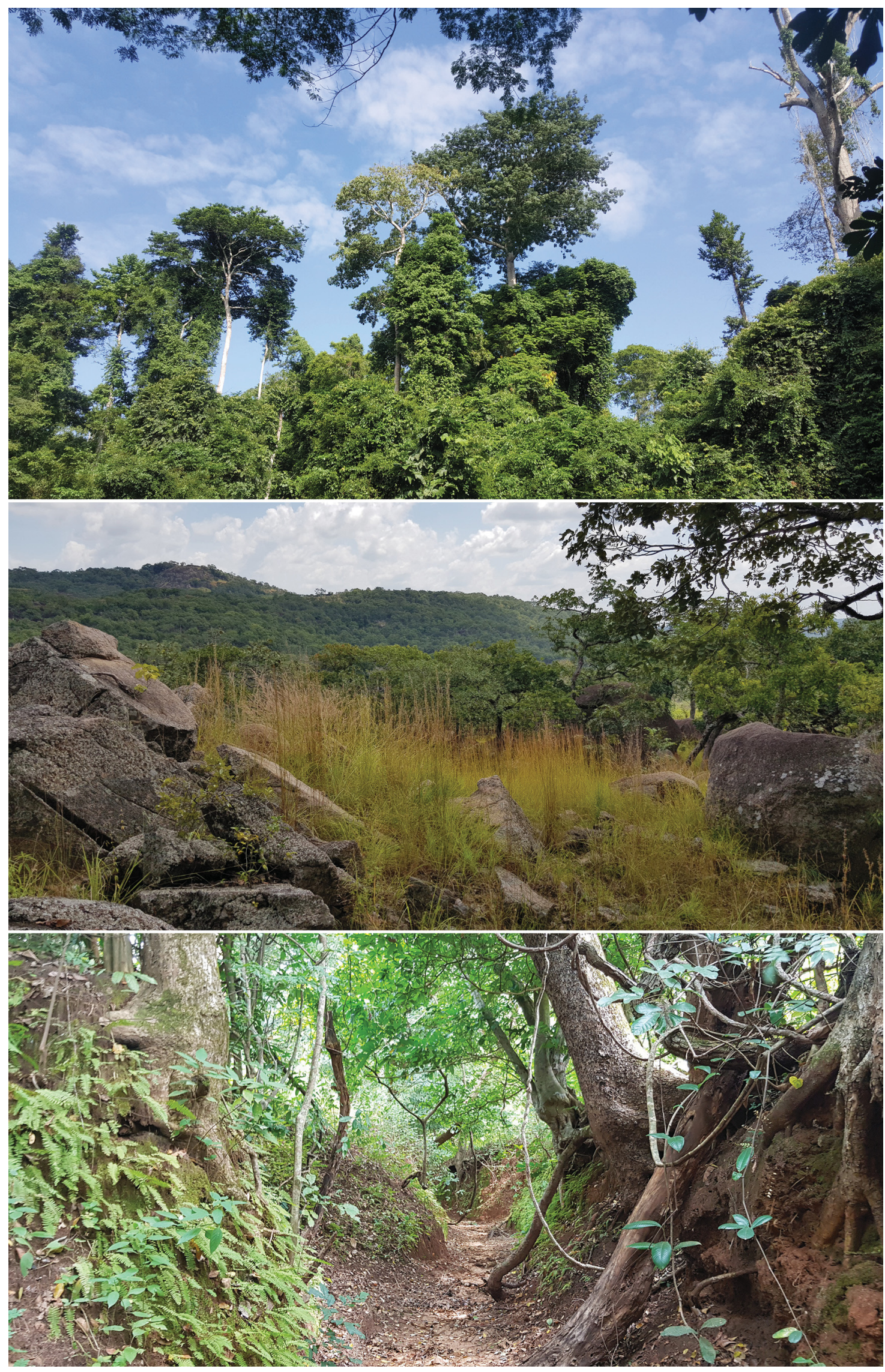

FIGS. 21-23. Côte d'Ivoire, Bossématié Forest, ecotone Guinean forest/Guinean forest-savanna mosaic, degraded mesophile forest (above); 2) Côte d'Ivoire, Denguélé Classified Forest (sudanian forest) (center); 3) Côte d'Ivoire, Denguélé Classified Forest, Gallery forest (bottom) (photos by P. Moretto). 


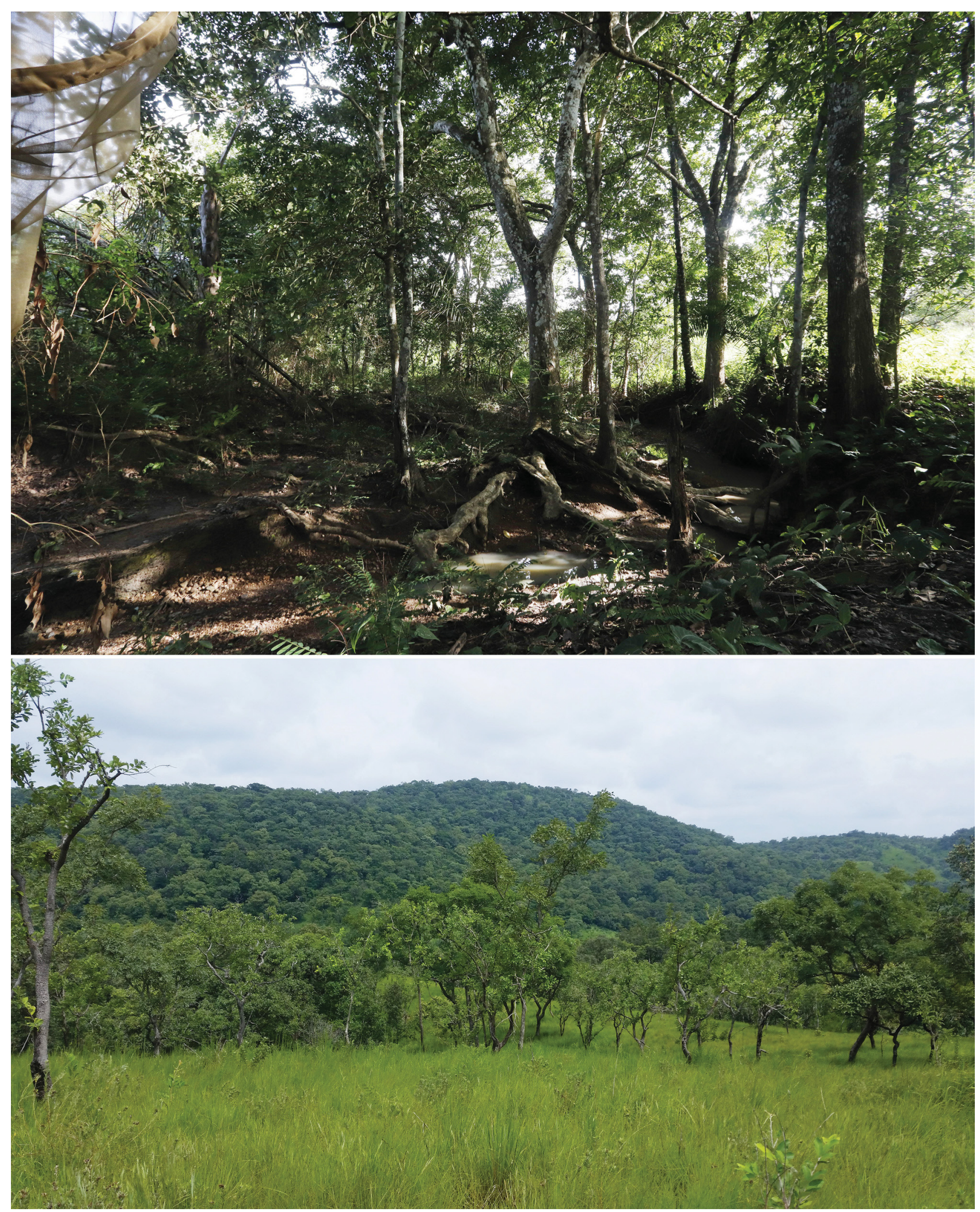

FIGS. 24-25. Togo, Fazao-Malfakassa National Park, gallery Forest (above) (photo by M. Aristophanous); Togo, Fazao-Malfakassa National Park, Mare aux crocodiles campsite, Sudanian savannah (bottom) (photo by P. Moretto).

\section{Togo (Figs. 24-25)}

The collecting sites were the following: 1) Togo, Fazao-Malfakassa NP, Point de vue campsite (Sudanian Savannah) (415m) 8`48’50”N, 0`49’3.2”E (MV, Lepiled, Actinic Light Trap) 16-23.VIII.2018, M. Aristophanous, M. 
Geiser, P. Moretto, B. Sanbena; 2) Togo, Fazao-Malfakassa NP, Mare aux crocodiles campsite (Sudanian Savannah/dry Forest) (505m) 8`44’58.8”N, 048’51.8”'E (MV, Actinic Light Trap) 26.VIII-7.IX.2018, M. Aristophanous, M. Geiser, P. Moretto, B. Sanbena; 3) Togo, Fazao-Malfakassa NP, Mare aux crocodiles campsite (Gallery Forest) (505m) 8`44'58.8”N, 048'51.8”'E (Lepiled Light Trap) 27-31.VIII.2018, M. Aristophanous, M. Geiser, P. Moretto, B. Sanbena; 4) Togo, Fazao-Malfakassa NP, Mare aux crocodiles campsite next to lake (Sudanian savannah) $8^{\circ} 44^{\prime} 58.8^{\prime}$ N, $0^{\circ} 48^{\prime} 51.8 ”$ 'E (505m) 5-7.IX.2018 (Lepiled Light Trap), M. Aristophanous, M. Geiser, P. Moretto, B. Sanbena.

They belong to the Ecotone West Sudanian savanna/Guinean forest-savanna mosaic. Mostly soudanian. The mountainsides and hillsides are covered with open forests with Isoberlinia and Uapaca and the tops of these hills, very eroded, occupied by open shrub savannas. The ravines can be occupied by true semi-deciduous gallery forests (in these areas typical forest Lepidoptera Rhopalocera live).

\section{Central African Republic (Figs. 26-27)}

Sampling of Orthoptera has been carried out in the Dzanga-Ndoki National Park, located in the extreme southwest of the Central African Republic, and in the Dzanga-Sangha Special Reserve. Dzanga-Ndoki NP is part of the Sangha Trinational, a UNESCO World Heritage Site which covers an area of 4,520,000 ha and includes three national Parks: Loboke (Cameroon), Nouabale-Ndoki (Congo) and Dzanga-Ndoki. It belongs to a north-western Congolian lowland forest, semi-deciduous diversified forest with Sterculiaceae-Ulmaceae, wide areas dominated by Gilbertiodendron dewevrei, and some humide zones along the rivers. For supplementary information see Massa et al. (2020).

\section{Results and Discussion}

\section{Annotated list of selected species}

Family Tettigoniidae Krauss, 1902

Subfamily Pseudophyllinae Burmeister, 1838

Tribe Pseudophyllini Burmeister, 1838

\section{Liocentrum aduncum Karsch, 1891}

Material examined. Liberia, Sinoe County, $6.5 \mathrm{~km}$ NW Jacksonville, Forest near Solve Problem Vill. (103m) 2327.I.2018 (MV Light Trap), M. Geiser, S. Safian, G. Simonics (1今̋); Guinea, Guinée forestière, Bossou For. \& Inst. Rech. Env. Bossou (Lowland Forest-Farmland) (690m) (MV Light Trap) 24-31.VI.2019, V. Dérozier, J. Suha Dore, S. Koivagui, W. Miles, S. Safian, R. Warner (1 +) (ANHRT); Côte d'Ivoire, Man, Mt. Tonkoui (1171m) 8.IX.2016


Biémasso (441m) 10-11.VII.2013 (light) (1 +), P. Moretto; Côte d'Ivoire, Comoe, Zamou 32.XI.2016 (light) (1 9 ), 21.V.2017 (light) (1ठ̂), P. Moretto; Côte d'Ivoire, Taï National Park, Res. Station (174m) 18.III.2017 (light), B.

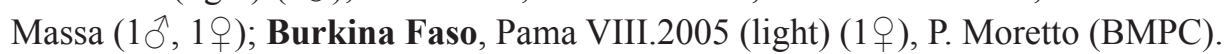

Distribution. Widespread in West Africa, recorded from Cameroon, Gabon, Democratic Republic of Congo, Guinea, Sierra Leone, and Liberia (Naskrecki 2008), now recorded also from Côte d'Ivoire.

\section{Liocentrum rubripes Bolívar, 1906 (Fig. 28)}

Material examined. Liberia, Lofa County, Wologizi Mts., Base Camp Forest (611m) (MV Light Trap) 20.XI1.XII.2017, M. Aristophanous, S. Safian, G. Simonics, L. Smith (2 + ) (ANHRT).

Distribution. Previously known from Cameroon and Guinea (Naskrecki 2008), now recorded also from Liberia. 



FIGS. 26-27. Central African Republic, Dzanga-Ndoki National Park, Ndoki forest (above); Central African Republic, DzangaNdoki National Park, clearings in front a lake (bottom) (photos by P. Moretto). 


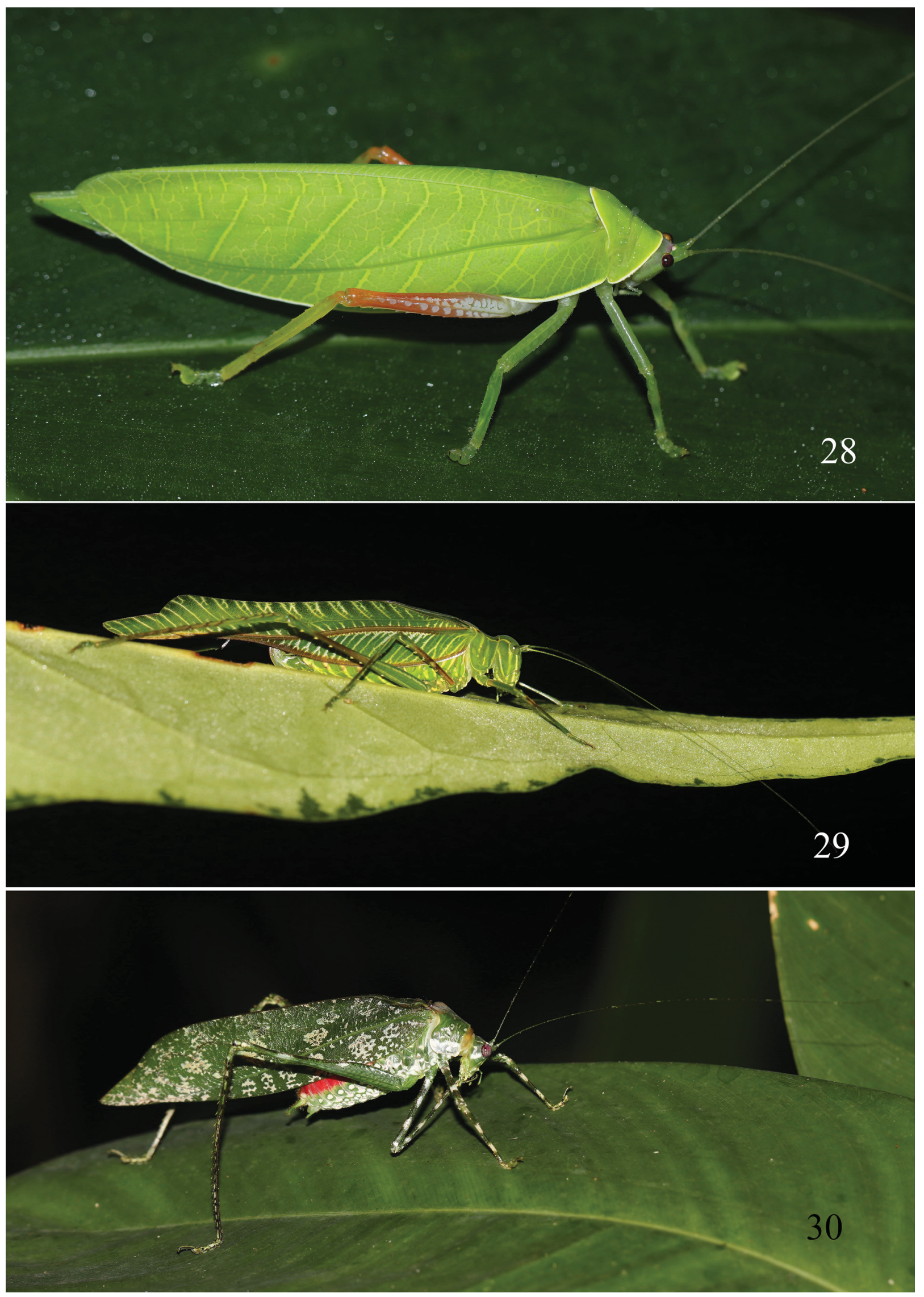

FIGS. 28-30. Liocentrum rubripes Bolívar, 1906, female from Liberia (28); Diogena fausta (Burmeister, 1838), female from Liberia (29); Arantia (Goetia) galbana (Karsch, 1891), male from Liberia, Foya Proposed Protected Area (30) (photos by M. Aristophanous). 
Material examined. Côte d'Ivoire, Mt. Tonkoui 1.XII.2015 (light), P. Moretto (1 +) (BMPC).

Distribution. Previously known only from the type material collected in Cameroon (Naskrecki 2008).

\section{Mustius superbus Sjöstedt, 1901}

Material examined. Liberia, Lofa County, Wologizi Mts., Base Camp Forest (611m) 20.XI-1.XII.2017 (MV Light Trap), M. Aristophanous, S. Safian, G. Simonics, L. Smith (1ㅇ) (ANHRT); Côte d'Ivoire, Man, Mt. Tonkoui (1171m) IIIIV.2017 (light) (1 +); Côte d'Ivoire, Mt. Tonkoui (1171m) IV.2017 (light), P. Moretto (1 P); Central African Republic, Dzanga-Sangha Special Reserve, Lac 1, 28-29.XI.2010, P. Annoyer (light) (1 P); Gabon, Mt. de Cristal 6-13.XI.2017, P. Le Gall (1 ㅇ) (BMPC).

Distribution. Previously known from Cameroon, Togo, Gabon, Equatorial Guinea (Fernando Poo), Democratic Republic of Congo, and Guinea (Naskrecki 2008), here recorded also from Liberia, Côte d'Ivoire, and Central African Republic.

\section{Mustius afzelii Stål, 1873}

Material examined. Central African Republic, Dzanga-Sangha Special Reserve, Mbaéré 17.XI.2010 (light), P. Annoyer (1) (BMPC).

Distribution. Known from Sierra Leone, Ghana, Cameroon and Democratic Republic of Congo (Naskrecki 2008), here recorded also from Central African Republic.

\section{Mustius eurypterus Karsch, 1896}

Material examined. Central African Republic, Dzanga-Sangha Special Reserve, Mbaéré, Lac 7, 17.XI.2010 (light), P. Annoyer (1 +) (BMPC); Côte d'Ivoire, Mt. Tonkoui (1171m) 10.VII.2020, 29.VII.2020 (light), P. Moretto (2ㅇ) (BMPC).

Distribution. Previously known from Cameroon, Democratic Republic of Congo and Guinea (Naskrecki 2008), now recorded also from Central African Republic, and Côte d'Ivoire.

\section{Desaulcya ampulla (Brunner von Wattenwyll, 1895)}

Material examined. Central African Republic, Dzanga-Sangha Special Reserve, Mbaéré 1.II.2012, 1.III.2012 (light), P. Annoyer $(1 \hat{\jmath}, 1$ ) $)$ (BMPC).

Distribution. Previously known only from the types from Gabon (Naskrecki 2008), here recorded also from Central African Republic.

\section{Opisthodicrus cochlearistylus Karsch, 1891}

Material examined. Liberia, Lofa County, Foya Proposed Protected Area (530m) 10-19.XI.2017 (MV Light Trap), M. Aristophanous, S. Safian, G. Simonics, L. Smith (1 +); Côte d'Ivoire, Mt. Tonkoui Peak (1171m) 9-16.IV.2016 (light Trap), M. Aristophanous, P. Moretto (2+); Côte d'Ivoire, Abidjan, Banco Forest (Banco PN) (39-48m) (MV Light Trap) 21-30.IV.2017, A. Aristophanous, M. Aristophanous, M. Geiser, P. Moretto (1 + ) (ANHRT); Côte

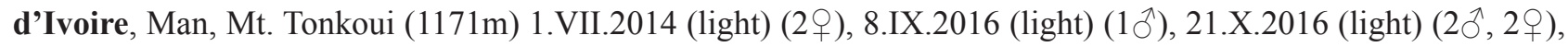
23.III.2017 (light) (1 +), III.IV.2017 (light) (1 +), 4.IV.2017 (light) (3 9 ), 23.V.2017 (light) (1 +), 19.VII.2017 (light) (1ㅇ), P. Moretto; Côte d'Ivoire, Taï National Park, Res. Station (174m) 18.III.2017 (light), B. Massa (3ð^, 5 ㅇ); Central African Republic, Dzanga-Ndoki Nat. Park, 20-23.II.2012 (light), P. Moretto (3 $\jmath^{\wedge}, 5$ ㅇ) (BMPC). 
Distribution. Previously known from Cameroon, Gabon, Angola, Equatorial Guinea (Fernando Poo), and Côte d'Ivoire (Naskrecki 2008), here recorded also from Liberia and Central African Republic.

\section{Zabalius lineolatus (Stål, 1873)}

Material examined. Togo, Fazao-Malfakassa NP, Point de vue campsite (Sudanian Savannah) (415m) 1623.VIII.2018 (Actinic Light Trap), M. Aristophanous, M. Geiser, P. Moretto, B. Sanbena (1ठ); Togo, Fazao-Malfakassa NP, Mare aux crocodiles campsite (Sudanian Savannah/dry Forest) (505m) 26.VIII-7.IX.2018 (MV Light Trap), M. Aristophanous, M. Geiser, P. Moretto, B. Sanbena (4ð̂, 3 + ); Côte d'Ivoire, Mt. Tonkoui Peak (1171m) 16-21.VII.2016 (light trap), M. Aristophanous, P. Moretto (1ð); Côte d'Ivoire, Taï National Park, Res. Station (174m) (MV Light Trap) 25.III-17.IV.2017, A. Aristophanous, M. Aristophanous, M. Geiser, P. Moretto (1ठ); Côte d'Ivoire, Taï National Park, Res. Station (174m) (Actinic Light Trap) 27.III-16.IV.2017, A. Aristophanous, M. Aristophanous, M. Geiser, P. Moretto (1ठ); Côte d'Ivoire, Taï National Park, Res. Station (174m) (Canopy Light Trap, 40m ab. ground) 27-31.III.2017, A. Aristophanous, M. Aristophanous, M. Geiser, P. Moretto (1ð, 1ㅇ) (ANHRT); Côte d'Ivoire, Man, Mt. Tonkoui (1171m) 8.VI.2017 (light) (1ð̋), 22.VII.2017, P. Moretto (1ठ); Côte d'Ivoire, Comoe, Kolomabira 11.XI.2016 (light), P. Moretto (1ð̋); Central African Republic, Dzanga-Ndoki Nat. Park, 2023.II.2012 (light), P. Moretto (3ð̄, 5 P) (BMPC).

Distribution. Known from Democratic Republic of Congo, Cameroon, Angola, Uganda, Guinea, Ghana, and Gabon (Naskrecki 2008, Massa 2021a), here recorded from Togo and Central African Republic.

\section{Tribe Phyllomimini Brunner von Wattenwyl, 1895}

\section{Rhinodera spinifrons Beier, 1955}

Material examined. Central African Republic, Dzanga-Sangha Special Reserve, Mbaéré 21.II.2012 (light), P. Annoyer (1) (BMPC).

Distribution. This very distinctive species is known only from the types, collected in Cameroon (Naskrecki 2008) and from one second record from Gabon (Massa 2021a); now it is recorded also from Central African Republic.

\section{Chondrodera subvitrea Karsch, 1891}

Material examined. Central African Republic, Dzanga-Ndoki Nat. Park, Ndoki 15-16.II.2012, P. Moretto (light) (19) (BMPC).

Distribution. Previously known only from the type material collected in Niger and Gabon, here recorded also from Central African Republic.

\section{Chondrodera ocellata Beier, 1954}

Material examined. Central African Republic, Dzanga-Sangha Special Reserve, Mbaéré 20.XI.2010, P. Annoyer (light) (1ठ̋); Côte d'Ivoire, Mt. Tonkoui (1171m), 7.XII.2017, 23.II.2020, 6.III.2020 (light) P. Moretto (3ㅇ) (BMPC).

Distribution. Known only from the type locality (Mundame, Cameroon), newly recorded from Central African Republic and Côte d'Ivoire.

\section{Chondrodera vittifer (Walker, 1871)}

Material examined. Côte d'Ivoire, Taï National Park, Res. Station 15.X.2018 (light), P. Moretto (1ð) (BMPC). Distribution. Described from Sierra Leone, recorded also from Democratic Republic of Congo (Naskrecki 2008); here reported also from Côte d'Ivoire. 
Material examined. Côte d'Ivoire, Taï National Park, Res. Station (light) 21.IX.2016, 20.III-4.IV.2017, 4.IX.2018, B. Massa, P. Moretto (2ð, 2q); Côte d'Ivoire, Zamou, Bondoukou VII.2004 (light), P. Moretto (1ठ̂); Côte d'Ivoire, Mt. Tonkoui (1171m) 1-4.VII.2014, 12-18.III.2015, 24-28.VII.2015, 12-16.II.2017, 1.IV.2017, 6.IV.2018, 8.VII.2019, 13-16.IX.2019 (light), P. Moretto (5今̄, 5 क ); Central African Republic, Dzanga-Ndoki National Park, Mbaéré 19-20.II.2012 (light), P. Moretto (1ㅇ) (BMPC).

Distribution. Previously known from Cameroon, Fernando Poo, Democratic Republic of Congo, and Côte d'Ivoire (Naskrecki 2008), now recorded also from Central African Republic.

\section{Stenampyx annulicornis Karsch, 1891}

Material examined. Sierra Leone, Tiwai Is., Moa River (120m) (light trap) 17-22.VI.2016, H. Takano, W. Miles, R. Goff (1 ); Liberia, Lofa County, Foya Proposed Protected Area (530m) 10-19.XI.2017 (MV Light Trap), M. Aristophanous, S. Safian, G. Simonics, L. Smith $(1 \hat{\jmath}, 1$ 1 $)$; Liberia, Sinoe County, Krahn-Bassa Reserve, Juboe River, 7.5 km SW Pellokon town, 14-21.I.2018 (MV Light Trap), M. Geiser, S. Safian, G. Simonics (19); Côte d'Ivoire, Yéalé Vill., Mt. Nimba, (380m) (light trap) 18-29.IV.2016, M. Aristophanous, M. Geiser, P. Moretto (2ᄋ); Côte d'Ivoire, Bossématié For. Classée (180m) (light trap) 25-31.VII.2016, M. Aristophanous, P. Moretto (19); Côte d'Ivoire, Taï National Park, Res. Station (174m) (MV Light Trap) 25.III-7.IV.2017, A. Aristophanous, M. Aristophanous, M. Geiser, P. Moretto (3ð̂, 3 ) ); Côte d'Ivoire, Taï National Park, Res. Station (174m) (Actinic Light Trap) 27.III-16.IV.2017, A. Aristophanous, M. Aristophanous, M. Geiser, P. Moretto (1ठ); Côte d'Ivoire, Abidjan, Banco Forest (Banco PN) (39-48m) (MV Light Trap) 21-30.IV.2017, A. Aristophanous, M. Aristophanous, M. Geiser, P. Moretto (4ð); Côte d'Ivoire, Mt. Tonkoui Peak (1171m) 3-7.V.2017 (MV Light Trap), A. Aristophanous, M. Aristophanous, M. Geiser, P. Moretto (1ठ); Côte d'Ivoire, Mt. Tonkoui Peak (1171m) 20-27.V.2018 (MV Light Trap), M. Aristophanous, W. Miles, P. Moretto, Y. Outtara (1 ) (ANHRT); Côte d'Ivoire, Man, Mt. Tonkoui

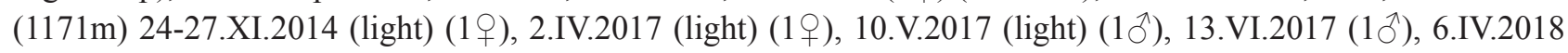


(light), P. Moretto (1); Côte d'Ivoire, Taï National Park, Res. Station 22.III-4.IV.2017 (light), P. Moretto (2q); Central African Republic, Dzanga-Ndoki Nat. Park, 20-23.II.2012 (light), P. Moretto (5^, 8ㅇ) (BMPC).

Distribution. Widespread in tropical Africa, known from Cameroon, Democratic Republic of Congo, Gabon, Sierra Leone, Fernando Poo, Guinea, Ghana, Côte d'Ivoire (Naskrecki 2008, Massa 2021a), now recorded also from Liberia and Central African Republic.

\section{Tomias (Tomias) stenopterus Karsch, 1891}

Material examined. Central African Republic, Dzanga-Sangha Special Reserve, Lac 1, 30.XI-1.XII.2010 (light), P. Annoyer (1 +); Central African Republic, Dzanga-Sangha Special Reserve, Mbaéré, Lac 1, 1-3.II.2012 (light), P. Annoyer (1 + ) (BMPC).

Distribution. Previously known from Cameroon, Equatorial Guinea, and Democratic Republic of Congo (Naskrecki 2008), here recorded also from Central African Republic.

Tribe Pleminiini Brunner von Wattenwyl, 1895

Adapantus (Adapantus) bardus Karsch, 1891

Material examined. Central African Republic, Dzanga-Ndoki National Park, 23-26.I.2012, 9-10.II.2012 (light), P. Moretto (3ठึ); Central African Republic, Dzanga-Sangha Special Reserve, Mbaéré Lac 1, 14-15.II.2012 (light), P. Annoyer (1ठ); Central African Republic, Dzanga-Sangha Special Reserve, Sangha river 10.X.2008 (light), P. Annoyer (1 $\left.\delta^{\Uparrow}\right)(\mathrm{BMPC})$. 
Distribution. Known from Cameroon and Ghana (Naskrecki 2008), here reported also from Central African Republic.

Adapantus (Adapantus) osorioi (Bolívar, 1886)

Material examined. Côte d'Ivoire, Taï National Park, Res. Station (174m) (MV Light Trap) 25.III-17.IV.2017, A. Aristophanous, M. Aristophanous, M. Geiser, P. Moretto (1ठ) (ANHRT).

Distribution. Known from Equatorial Guinea and Cameroon (Naskrecki 2008), here recorded also from Côte d'Ivoire.

Adapantus (Neoadapantus) affluens Naskrecki, 2008

Material examined. Côte d'Ivoire, Taï National Park, Res. Station (174m) (MV Light Trap) 25.III-17.IV.2017, A. Aristophanous, M. Aristophanous, M. Geiser, P. Moretto (1ठ); Côte d'Ivoire, Taï National Park, Res. Station (174m) (Canopy Light Trap, 40m ab. ground) 27-31.III.2017, A. Aristophanous, M. Aristophanous, M. Geiser, P. Moretto (1§) (ANHRT); Côte d'Ivoire, Mt. Tonkoui (1171m) 28.VI-1.VII.2014, 13-15.VII.2014, 24-27.XI.2014, 14.XI.2018, 16.IX.2019, 2.XII.2019, 25.VII.2020 (light), P. Moretto (7ठ) (BMPC).

Distribution. Described from Guinea (Naskrecki 2008), here recorded also from Côte d'Ivoire.

Adapantus (Neoadapantus) angulatus Naskrecki, 2008

Material examined. Côte d'Ivoire, Taï National Park, Res. Station (174m) (MV Light Trap) 25.III-17.IV.2017, A. Aristophanous, M. Aristophanous, M. Geiser, P. Moretto (1ठ) (ANHRT); Côte d'Ivoire, Taï National Park, Res. Station (174m) 22.III-4.IV.2017 (light), P. Moretto (4§̋); Côte d'Ivoire, Korhogo, Koko Vill., 13-15.VII.2014 (light), P. Moretto (1へ̂) (BMPC).

Distribution. Described from Guinea (Naskrecki 2008), now recorded also from Côte d'Ivoire.

\section{Adapantus (Neoadapantus) marmoratus Chopard, 1954}

Material examined. Côte d'Ivoire, Mt. Tonkoui (1171m) 16.IX.2019 (light), P. Moretto (1 $\overbrace{}^{\Uparrow})$ (BMPC).

Distribution. Known from Guinea and Ghana (Naskrecki 2008), here recorded also from Côte d'Ivoire.

\section{Adapantus (Neoadapantus) nitens (Chopard, 1954)}

Material examined. Côte d'Ivoire, Taï National Park, Res. Station (174m) (light) 21.III-17, 4.IV.17, B. Massa, P. Moretto (5今); Côte d'Ivoire, Mt. Tonkoui (1171m) 28.VI-1.VII.2014, 6.XII.2018, 22.I.2019, 15.II.2019, 4.VII.2019, 3-12.XI.2019, 4.IV.2020, 30.XII.2020 (light), P. Moretto (8ð̂, 2q); Côte d'Ivoire, Korhogo, Koko Vill., 13-15.VII.2014 (light), P. Moretto (1§); Côte d'Ivoire, Biémasso 10-11.VII.2013, P. Moretto (1ठ); Côte d'Ivoire, Sassandra XII.1996 (light), P. Moretto (10̂, 2ㅇ) (BMPC).

Distribution. Previously known from Guinea and Ghana (Naskrecki 2008), here recorded also from Côte d'Ivoire.

\section{Mormotus alonsae Naskrecki, 2008}

Material examined. Côte d'Ivoire, Taï National Park, Res. Station (174m) (light) 18.III-17, B. Massa (1ð̂); Côte d'Ivoire, Mt. Tonkoui (1171m) 24-27.XI.2014, 1.III.2019 (light), P. Moretto (2§); Côte d'Ivoire, Korhogo, Koko vill. 13-15.VII.2014 (light), P. Moretto (1今) (BMPC).

Distribution. Described from Guinea by Naskrecki (2008), now recorded from Côte d'Ivoire. 
Material examined. Liberia, Lofa County, Wologizi Mts., Base Camp Forest (611m) 20.XI-1.XII.2017 (MV Light Trap), M. Aristophanous, S. Safian, G. Simonics, L. Smith (1ð̋); Guinea, Ditinn, Chute de Ditinn (771m) (Lepiled Light Trap) 18-25.IX.2019, M. Geiser, M. Leno, S. Koivagui, W. Miles, L. Mulvaney, S. Safian (1 9 ) (ANHRT); Côte d'Ivoire, Mt. Tonkoui (1171m) 15.IV.2019 (light), P. Moretto (1ð̋) (BMPC).

Distribution. Previously known from Côte d'Ivoire and Ghana (Naskrecki 2008), here recorded also from Liberia and Guinea.

\section{Mormotus clavaticercus Karsch, 1891}

Material examined. Liberia, Lofa County, Wologizi Mts., Base Camp Forest (611m) 20.XI-1.XII.2017 (MV Light Trap), M. Aristophanous, S. Safian, G. Simonics, L. Smith (1ð̋ parasitized, 1ㅇ); Côte d'Ivoire, Abidjan, Banco Forest (Banco PN) (39-48m) (MV Light Trap) 21-30.IV.2017, A. Aristophanous, M. Aristophanous, M. Geiser, P. Moretto (3 $\widehat{O}^{\Uparrow}$ ); Côte d'Ivoire, Abidjan, Banco Forest (Banco PN) (39-48m) (gen. coll.) 21.IV-1.V.2017, A. Aristophanous, M. Aristophanous, M. Geiser, P. Moretto (1ð̄, 2ㅇ) (ANHRT); Côte d'Ivoire, Taï National Park, Res. Station 23.III.2017 (light), P. Moretto (1ð̋); Côte d'Ivoire, Taï National Park, Res. Station 21.III-4.IV.2017 (light), P. Annoyer, B. Massa, P. Moretto (4ठ̊); Côte d'Ivoire, Mt. Tonkoui, 24-27.XI.2014, 20.VI.2015, 12-16.III.2017, 31.X.2018, 14.XI.2018, 22.XII.2018, 5.I:2019, 3.VI.2019, 30.VII.2019 (light), P. Moretto (11 $\sigma^{\lambda}, 3$ P ) (BMPC).

Distribution. Previously known from Sierra Leone, Guinea, Liberia and Ghana (Naskrecki 2008), here recorded also from Côte d'Ivoire.

\section{Mormotus montesi (Bolívar, 1886)}

Material examined. Liberia, Lofa County, Wologizi Mts., Base Camp Forest (611m) 20.XI-1.XII.2017 (MV Light Trap), M. Aristophanous, S. Safian, G. Simonics, L. Smith (1+1); Côte d'Ivoire, Abidjan, Banco Forest (Banco PN) (39-48m) 5²3'03.8'N, 403'11.2”W (MV Light Trap) 21-30.IV.2017, A. Aristophanous, M. Aristophanous, M.

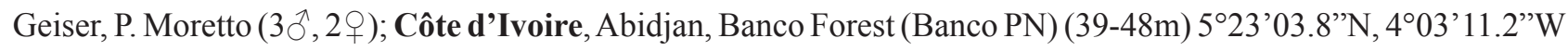
(gen. coll.) 21.IV-1.V.2017, A. Aristophanous, M. Aristophanous, M. Geiser, P. Moretto (3 + ) (ANHRT); Côte d'Ivoire, Biémasso (441m) 7.VII.2014 (light), P. Moretto (2)); Côte d'Ivoire, Taï National Park, Res. Station 1321.III.2017 (light), B. Massa (5 +); Ghana, Aburi VI.1984 (1ð), XI.1984 (1 ), P. Butti; Central African Republic, Dzanga-Ndoki National Park, Ndoki camp 1, 20-23.II.2012 (light), P. Moretto (1ㅇ) (BMPC).

Distribution. Previously known from Guinea, Cameroon, Equatorial Guinea (Fernando Poo), and Democratic Republic of Congo (Naskrecki 2008), here recorded also from Liberia, Côte d'Ivoire, Ghana and Central African Republic.

\section{Habrocomes lanosus Karsch, 1891}

Material examined. Côte d'Ivoire, Taï National Park, Res. Station (174m) 21.III-4.IV.2017 (light), B. Massa, P. Moretto (2ð) (BMPC).

Distribution. Known from Sierra Leone, Guinea and Ghana, here reported also from Côte d'Ivoire.

\section{Habrocomes personatus personatus (Sjöstedt, 1901)}

Material examined. Central African Republic, Dzanga-Ndoki Nat. Park, Lac 1, 20-23.II.2012 (light), P. Moretto

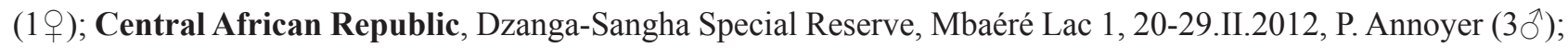
Central African Republic, Dzanga-Sangha Special Reserve, Mbaéré Lac 3, 25-26.II.2012 (light), P. Annoyer (1 ${ }^{\lambda}$ ) (BMPC). 
Distribution. Previously known only from Cameroon (Naskrecki 2008), now recorded also from Central African Republic.

\section{Tympanocompus acclivis Karsch, 1891}

Material examined. Central African Republic, Dzanga-Ndoki Nat. Park, Lac 7, 2.II.2012 (light), P. Moretto (1ठ); Central African Republic, Bambio (490m) 10.XII.2008, J. Halada (1ठ); Central African Republic, DzangaSangha Special Reserve, Mbaéré, Lac 7, 28-29.II.2012, P. Annoyer (1ðึ); Central African Republic, DzangaSangha Special Reserve, Camp 2, 19-20.X.2008 (light), P. Annoyer (1§); Central African Republic, DzangaSangha Special Reserve, between Boda and N'Gotto 20.I.2005, P. Annoyer (1 đ); Gabon, Mt. de Cristal National Park, Kinguelé 2.XII.2015, N. Moulin; Côte d'Ivoire, Aszani National Park (59m) 26.XI-1.XII.2015, M. Aristophanous, P. Moretto, E. Ruzzier (1ठ) (BMPC).

Distribution. Previously known from Cameroon, Equatorial Guinea and Guinea-Bissau (Naskrecki 2008), now recorded also from Central African Republic, Gabon and Côte d'Ivoire.

\section{Hoplidostylus argillatus Karsch, 1893}

Material examined. Côte d'Ivoire, Comoe, Kolomabira (228m) 19.XI. 2014 (light), P. Moretto (1 $\overbrace{}^{\Uparrow})$ (BMPC).

Distribution. Previously known only from the type collected in Togo (Naskrecki 2008), now recorded from Côte d'Ivoire.

\section{Lichenochrus crassipes Krasch, 1890}

Material examined. Ghana, Aburi I.1985, P. Butti (1ठ); Côte d'Ivoire, Mt. Tonkoui 12-16.III.2017, 30.III.2019 (light), P. Moretto (1ठ, 1 1 ); Côte d'Ivoire, Taï National Park, Res. Station (174m) 20.III-4.IV.2017 (light), B. Massa, P. Moretto (2ð) (BMPC).

Distribution. Known from several localities of Cameroon and Guinea (Naskrecki 2008), now recorded also from Côte d'Ivoire and Ghana.

\section{Lichenochrus marmoratus Sjöstedt, 1902}

Material examined. Côte d'Ivoire, Taï National Park, Res. Station (174m) 20.III-4.IV.2017 (light), P. Moretto $(1 \precsim)$ (BMPC).

Distribution. Previously known from Cameroon (Naskrecki 2008), now recorded also from Côte d'Ivoire.

\section{Batodromeus richardi (Griffini, 1908)}

Material examined. Côte d'Ivoire, Mt. Tonkoui Peak (1171m) 16-21.VII.2016 (light trap), M. Aristophanous, P. Moretto (1ठ); Côte d'Ivoire, Mt. Tonkoui Peak (1171m) 19-27.XI.2019 (MV light Trap), M. Aristophanous, V. Dérozier, P. Moretto, S. Ouattara (1ठ); Côte d'Ivoire, Taï National Park, Res. Station (174m) (MV Light Trap) 25.III-17.IV.2017, A. Aristophanous, M. Aristophanous, M. Geiser, P. Moretto (1 $\overbrace{}^{\Uparrow})$ (ANHRT); Côte d'Ivoire, Mt. Tonkoui 16-17.IX.2018, 26.IX.2019, 2.XII.2019 (light), P. Moretto (1ठึ, 2 q); Côte d'Ivoire, Taï National Park, Res. Station (174m) 18.III.2017 (light), B. Massa (1 $\overbrace{}^{\Uparrow})$ (BMPC).

Distribution. Described from Côte d'Ivoire (Griffini 1908) on a female sex, later described the male from Ghana (Naskrecki 2008), here recorded males and females from the hotspots Mt. Tonkoui and Taï National Park, Côte d'Ivoire. 
Material examined. Côte d'Ivoire, Mt. Tonkoui 24-27.XI.2014 (light), 12-18.VII.2015 (light), 12-16.III.2017

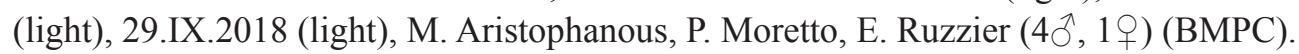

Distribution. Previously known from Ghana and Sierra Leone (Naskrecki 2008), now recorded also from the hotspot Mt. Tonkoui, Côte d'Ivoire.

\section{Subfamily Conocephalinae Burmeister, 1838}

\section{Tribe Copiphorini Karny, 1912}

\section{Lanista africana (Walker, 1871)}

Material examined. Sierra Leone, Loma Mts., Closed-canopy for. (1050m) (light trap) 7-10.VI.2016, H. Takano, W. Miles, R. Goff (1 ) ); Côte d'Ivoire, Mt. Tonkoui Peak (1171m) 9-16.IV.2016 (light Trap), M. Aristophanous, P. Moretto (1 ); Côte d'Ivoire, Mt. Tonkoui Peak (1171m) 20-27.V.2018 (MV Light Trap), M. Aristophanous, W. Miles, P. Moretto, Y. Outtara (1 $\widehat{\delta}^{\text {) }}$ (ANHRT); Côte d'Ivoire, Lamto Res. Station, Bandama river 3.VII.1962 (light) (19); same locality 24.VII.1962 (light) (19); Côte d'Ivoire, Man, Mt. Tonkoui (1171m) 19.II.2017 (light) (19),



Distribution. Naskrecki \& Guta (2019) have reinstated this taxon, which results to be present only in West Africa (Guinea and Ghana); here it is recorded also from Sierra Leone and Côte d'Ivoire.

\section{Pseudorhynchus raggei nomen novum}

Material examined. Côte d'Ivoire, Mt. Tonkoui (1170m) 30.X.2015, 28.IX.2019, 5.X.2019 (light), P. Moretto (3ㅇ); Côte d'Ivoire, Comoe, Zamou (245m), 16.XII.2016 (light), P. Moretto (19); Guinea, Mt. Nimba, Bakoro Camp, 26.IX.2008 (2+) (BMPC).

Pseudorhynchus raggei nomen novum is a replaced name for Pseudorhynchus robustus Ragge, 1969 (types from Sierra Leone), which results to be a junior primary homonym of Pseudorhynchus robustus Willemse, 1953 (types from Solomon Is., Papuasia). In his revision of the genus Pseudorhynchus, the English orthopterist Ragge (1969) inadvertently described P. robustus, a preoccupied name, for a species occurring widely in West and Central Africa. Pseudorhynchus raggei nomen novum is dedicated to the distinguished David Ragge, author of the monograph on Pseudorhynchus and many other excellent papers on tropical African Tettigoniidae and Orthoptera bioacoustics.

\section{Pseudorhynchus pungens werneri Karny, 1907}

Material examined. Senegal, Kolda XI.2009 (light), P. Moretto (1§); Senegal, Kedougou (light) VII.2008, P.

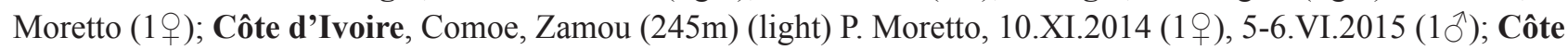
d'Ivoire, Comoe, Kolomabira (228m) 13.VII.2015 (light), P. Moretto (1今̂, 1 1 +); Côte d'Ivoire, Boundiali, M'Banto (395m) 13-15.VII.2015 (light), P. Moretto (1 + ); Côte d'Ivoire, Mt. Tonkoui (1171m) 3.XII.2016 (light), P. Moretto $\left(1{ }^{\wedge}\right)$ (BMPC).

Remarks. P. pungens (Schaum, 1853) is widespread in all the tropical Africa; according to Ragge (1969), P. pungens werneri covers West and Central Africa, as far as southern Sudan. However, as suggested by Naskrecki \& Guta (2019), it may represent only a case of color polymorphism, mainly if we consider the wide distribution of the species. 


\section{Tribe Phaneropterini Burmeister, 1838}

\section{Phaneroptera maculosa Ragge, 1956}

Material examined. Liberia, Sinoe County, 6.5 km NW Jacksonville, Forest near Solve Problem Vill. (103m) 2327.I.2018 (MV Light Trap), M. Geiser, S. Safian, G. Simonics (19) (ANHRT).

Distribution. P. maculosa was previously known from Cameroon, Central African Republic, Democratic Republic of Congo (Ragge 1980, Massa 2013), here it is recorded also from Liberia.

\section{Tribe Amblycoryphini Brunner von Wattenwyl, 1878}

\section{Genus Plangia Stål, 1873}

The genus Plangia is richer in species than it is generally believed. Recently Hemp et al. (2015) and Hemp (2017) have revised the Plangia graminea complex in East Africa, finding that P. graminea (Serville, 1838) is very probably only distributed in southern Africa, while four previously unknown species, P. satiscaerulea Hemp, 2015 (Mt. Kilimanjaro), P. multimaculata Hemp, 2015 (Mt. Kilimanjaro), P. amaniensis Hemp, 2017 (Usambara Mts.) and $P$. variacantans Hemp, 2017 (Uluguru Mts.) are present in Tanzania. Previous authors highlighted that very probably in West Africa other species are the representatives of the P. graminea complex. Material collected in central and west tropical Africa allowed to highlight the high species diversity in the genus Plangia also in those forests.

Massa (2021a) reported two unidentified species of Plangia from Zambia and Gabon; they will be discussed in another paper.

\section{Plangia astylata n. sp. (Figs. 31a-d)}

Material examined. Central African Republic, Dzanga-Sangha Special Reserve, Camp 1, 27-28.I.2005 (light), P.

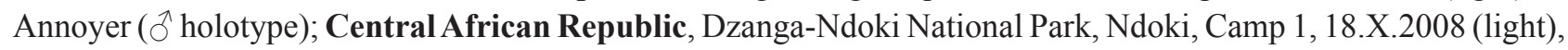
P. Annoyer (1ठ paratype); Central African Republic, Dzanga-Sangha Special Reserve, Lac 1, 23-24.XI.2010 (light), P. Annoyer (1 $\hat{\delta}^{\lambda}$ paratype) (BMPC).

Diagnosis. It is a small Plangia, characterized by the absence of styli, cerci long, incurved and very pointed. It was listed as Plangia sp.1 by Massa et al. (2020).

Description. Male. Colour. Yellow-green, abdomen and legs brown; cerci black-tipped. Head and antennae. Fastigium of vertex as wide as scapus, not contiguous with the fastigium of frons. Face smooth. Thorax and legs. Anterior margin of pronotum incurved, posterior margin rounded. Fore coxae armed with a big spine. Fore femora short and compressed, with 4 spines on the inner ventral margin, fore tibiae a little compressed at the base, short, with open tympana, sulcate above, with 2 outer and inner ventral spines +1 apical spur on each side, and 1 outer dorsal spur. Mid femora with 3 spines on outer ventral margin, mid tibiae with 4 outer and inner ventral spines +1 apical spur on each side and 1 inner dorsal spur. Hind femora just compressed, wrinkled, with 3 spines on outer ventral margin, hind tibiae with 11 outer and inner dorsal spines and 10 outer and inner ventral spines +3 apical spurs on each side. Wings. Tegmina 3.2-3.3 times longer than wide, with fore and hind margins more or less parallel, the fore margin only apically rounded. Stridulatory area of the left tegmen well raised, mirror on the right tegmen triangular (Fig. 31a), stridulatory file under the left tegmen arched and consisting of ca. 140 teeth more or less regularly spaced, their width decreasing from inner to outer margin (Fig. 31d). Abdomen. Cerci regularly decreasing in size, incurved and very pointed (Fig. 31b); subgenital plate triangular, styli absent, two small processes similar to the styli present at the apex of the plate (Fig. 31c).

Measurements (mm). Male. Body length: 16.9-17.2; pronotum length: 4.3-4.4; length hind femur: 10.2-10.6; tegmina: 24.5-26.4; tegmina width: 8.0-8.1.

Etymology. The name astylata derives from the Latin language and means 'without styli' (privative alpha + abjective feminine stylata). 
Affinities. The species of the genus Plangia generally have small or tiny styli (e.g. P. deminuta, P. karschi, P. nebulosa, etc.; but $P$. variacantans has unusually long styli: Hemp 2017); however, presently species without styli are not known; further, the cerci of the new species are a little similar to those of P. graminea.

Distribution. Presently P. astylata n. sp. is known only from Central African Republic (Dzanga-Ndoki National Park and Dzanga-Sangha Special Reserve).

\section{Plangia chopardi n. sp. (Figs. 32a-g)}

Material examined. Côte d'Ivoire, Abidjan, Banco Forest (Banco PN) (39-48m) (MV Light Trap) 21-30.IV.2017, A. Aristophanous, M. Aristophanous, M. Geiser, P. Moretto (1 $\delta^{\lambda}$ holotype, 1 ㅇ paratype) (ANHRT).

Diagnosis. Plangia chopardi $\mathrm{n}$. sp. is characterized by its longitudinal brown stripes on the pronotum disc and by the stout and long ovipositor.

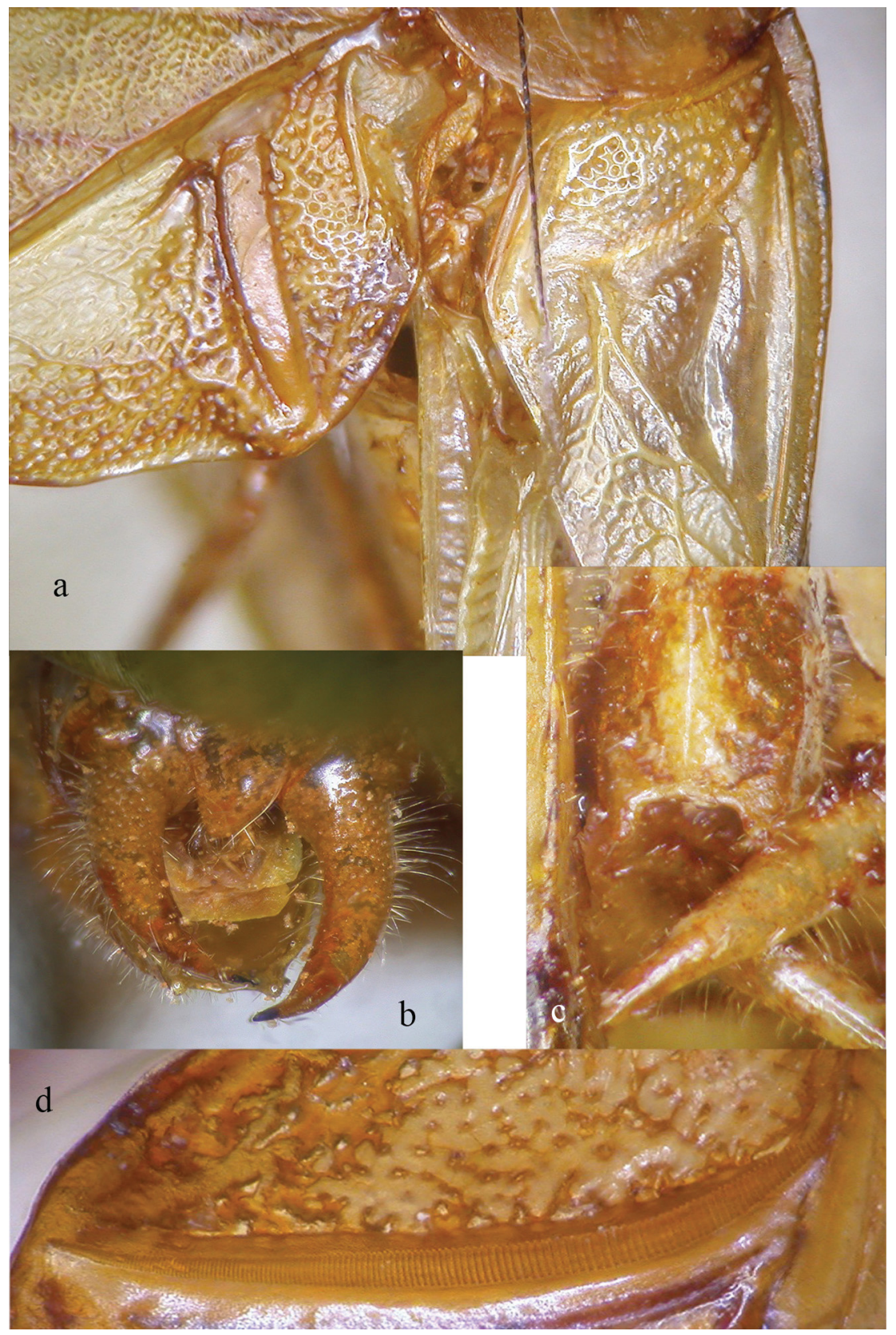

FIG. 31. Plangia astylata n. sp.: a) dorsal view of left and right tegmina of the male; b) dorsal view of male cerci; c) ventral view of the male subgenital plate; d) stridulatory file under the male left tegmen. 




FIG. 32. Plangia chopardi n. sp.: a) habitus of the female; b) dorsal view of male head, pronotum and tegmina; c) stridulatory file under the male left tegmen; d) dorsal view of male cerci; e) ventral view of the male subgenital plate; f) female ovipositor; g) ventral view of the female subgenital plate; Plangia karschi Chopard, 1954: h) dorsal view of male head, pronotum and tegmina. 



FIG. 33. Plangia karschi Chopard, 1954: a) habitus of the male; b) habitus of the female; c) dorsal view of male cerci and styli; d) ventral view of the male subgenital plate; e) stridulatory file under the male left tegmen. 
Description. Male. Colour. Yellow-green, with scattered greyish spots on tegmina, pronotum disc with longitudinal brown stripes, tibiae brown with white spots, hind tibiae with black-tipped spines (Figs. 32a-b). Head and antennae. Fastigium of vertex just wider than scapus, not contiguous with the fastigium of frons. Face smooth. Thorax and legs. Anterior margin of pronotum straight, posterior margin rounded. Longitudinal brown stripes on the pronotum disc (Fig. 32b). Fore coxae armed with a big spine. Fore femora short and compressed, with 4 spines on the inner ventral margin, fore tibiae a little compressed at the base, with open tympana, sulcate above, with 3 outer and inner ventral spines +1 apical spur on each side, and 1 outer dorsal spur. Mid femora with 4 spines on outer ventral margin, mid tibiae with 4 outer and inner ventral spines +1 apical spur on each side and 1 inner dorsal spur. Hind femora just compressed, wrinkled, with 5 spines on outer ventral margin, hind tibiae with 11 outer and inner dorsal spines and 8 outer and inner ventral spines +3 apical spurs on each side. Wings. Tegmina 3.6 times longer than wide, with fore and hind margins more or less parallel, the fore margin only apically rounded. Stridulatory area of the left tegmen just raised, mirror on the right tegmen triangular (Fig. 32b), stridulatory file under the left tegmen arched and consisting of ca. 60 teeth regularly spaced (Fig 32c). Abdomen. Cerci stout and in-curved (Figs. 32d-e); subgenital plate triangular, styli small (Fig. 32e).

Female. Same characters as the male (Fig. 32a), length/width tegmina 3.2, ovipositor very big for the genus Plangia, gently up-curved, with many small teeth dorsally and a few teeth ventrally at the apex (Fig. 32f). Subgenital plate triangular and pointed (Fig. 32g).

Measurements (mm). Male. Body length: 23.4; pronotum length: 4.5-5.5; length hind femur: 13.5-16.0; tegmina: 29.0-33.5; tegmina width: 8.0-9.7. Female. Body length: 23.4; pronotum length: 6.8; length hind femur: 17.6; tegmina: 35.3 ; tegmina width: 11.0 ; ovipositor: 8.9 .

Etymology. Plangia chopardi $\mathrm{n}$. sp. is dedicated to Lucien Chopard (31 August 1885-16 November 1971), a distinguished French entomologist, who contributed very much to the knowledge of African orthopterofauna, describing many species of Orthoptera, also belonging to the genus Plangia.

Affinities. No other Plangia species have the combination of characters of $P$. chopardi n. sp.: longitudinal brown stripes on the pronotum disc, stridulatory file of ca. 60 regularly spaced teeth, and stout and long ovipositor.

Distribution. P. chopardi n. sp. is presently known from Côte d'Ivoire.

Habitat. Eastern Guinean forest, humide psammohygrophile forest characterized by Turaeanthus africanus and Heisteria parvifolia, primary forest and old secondary forest, characerized by Lophira alata.

\section{Plangia karschi Chopard, 1954 (Figs. 32h, 33a-e)}

Material examined. Togo, Fazao-Malfakassa NP, Point de vue campsite (Sudanian Savannah) (415m) 1623.VIII.2018 (MV Light Trap), M. Aristophanous, M. Geiser, P. Moretto, B. Sanbena (1ठ̂); Togo, Fazao-Malfakassa NP, Mare aux crocodiles campsite (Sudanian Savannah/dry Forest) (505m) 26.VIII-7.IX.2018 (MV Light Trap), M. Aristophanous, M. Geiser, P. Moretto, B. Sanbena (1ðß); Côte d'Ivoire, Mt. Tonkoui Peak (1171m) 2027.V.2018 (MV Light Trap), M. Aristophanous, W. Miles, P. Moretto, Y. Outtara (1 ${ }_{\widehat{C}}$ ); Côte d'Ivoire, Dolla Ranch (tree savannah) (481m) (MV Light trap) 27.V-5.VI.2018, M. Aristophanous, W. Miles, P. Moretto, Y Outtara (1ㅇ)

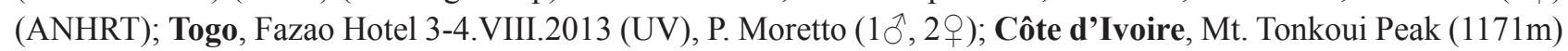

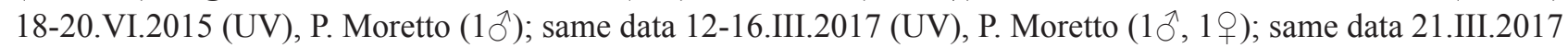
(UV), P. Moretto (2ᄋ); same data 7.IV.2018 (UV), P. Moretto (1ठ); same data 4.VI.2018 (UV), P. Moretto (1); Côte d'Ivoire, Comoe, Zamou (245m) 5-6.VI.2015 (UV), P. Moretto (29); same data 2.XI.2016 (UV), P. Moretto (1ठ); Côte d'Ivoire, Comoe, Kolomabira (228m) 11.VII.2015 (UV), P. Moretto (1ㅇ); Côte d'Ivoire, Dabakalla, Ouanderama (242m) 22-27.V.2016 (UV), P. Moretto (1 9 ) (BMPC).

Remarks. Chopard (1954) described only the female of this species; the series of males above listed were considered belonging to $P$. karschi because they were collected together females ascribable to this species. Chopard (1954) wrote that tegmina are 3.5 times longer than wide, but the measurements of the female reported by him indicate 3.8 times longer than wide. Only small differences from the general shape of the female described by Chopard (1954) were noticed. Plangia karschi is one of the complex of species characterized by the black spot on the male stridulatory area of the left tegmen, which very probably replaces $P$. graminea in West Africa.

Description of the male. Colour. Yellow-green, a black spot on the stridulatory area of the left tegmen; only in some females small scattered brown spots on tegmina (Figs. 32h, 33a-b). Head and antennae. Fastigium of vertex 
as wide as scapus, not contiguous with the fastigium of frons. Face smooth. Thorax and legs. Anterior margin of pronotum a little incurved, posterior margin rounded. Fore coxae armed with a big spine. Fore femora short and compressed, with 3 spines on the inner ventral margin, fore tibiae a little compressed at the base, with open tympana, sulcate above, with 2-3 outer and inner ventral spines +1 apical spur on each side, and 1 outer dorsal spur. Mid femora with 3 spines on outer ventral margin, mid tibiae with 3-4 outer and inner ventral spines +1 apical spur on each side and 1 inner dorsal spur. Hind femora just compressed, externally wrinkled, with 5 spines on outer ventral margin, the outer genicular lobe with a small spine, hind tibiae with 11-12 outer and inner dorsal spines and 10-11 outer and 5-7 inner ventral spines +3 apical spurs on each side. Wings. Tegmina 3.5-3.8 times longer than wide, with fore and hind margins more or less parallel, the fore margin only apically rounded. Stridulatory area of the left tegmen just raised, mirror on the right tegmen triangular (Fig. 32h), stridulatory file under the left tegmen arched and consisting of more than 80 teeth regularly spaced, in the middle larger than at both ends (Fig. 33e). Abdomen. Cerci stout and in-curved, apically tipped (Fig. 33c); subgenital plate triangular, styli small (Fig. 33d).

Measurements (mm). Males. Body length: 19.0-24.5; pronotum length: 4.5-5.5; length hind femur: 13.5-16.0; tegmina: 29.0-33.5; tegmina width: 8.0-9.7. Females (in parenthesis after Chopard 1954). Body length: 17.2-24.7 (18.0); pronotum length: 4.2-5.6 (4.6); length hind femur: 10.4-15.5 (16.0); tegmina: 26.0-34.5 (27); tegmina width: 8.2-10.0 (7.1); ovipositor: 4.3-6.5 (4.5).

Affinities. The male of $P$. karschi differs from $P$. graminea and $P$. satiscaerulea by the shape of cerci and the number of teeth in the stridulatory file, from P. astylata n. sp. by the stridulatory file (ca. 140 teeth) and the absence of styli. The male of $P$. villiersi is unknown, but female tegmina are fairly oval.

Distribution. Chopard (1954) described the female of this species from Mt. Nimba, N'zo and Kéoulenta (Guinea). Now it is recorded also from Togo and Côte d'Ivoire.

\section{Plangia villiersi Chopard, 1954}

Material examined. Côte d'Ivoire, Mt. Tonkoui (1171m) 12-16.III.2017 (UV), P. Moretto (2ᄋ); Côte d'Ivoire, Taï National Park, Res. Station, 27.III-4.IV.2017 (UV), P. Moretto (1 +); Gabon, Lope National Park, 13-14.III.2013, N. Moulin (19).

Measurements (mm). Females (in parenthesis after Chopard 1954). Body length: 17.4-20.9 (20.5); pronotum length: 5.1-5.2 (5.5); length hind femur: 12.0-13.5 (17.0); tegmina: 27.6-29.0 (32.0); tegmina width: 10.6-11.0 (11.6); ovipositor: 7.5-7.6 (5.0).

Distribution. Plangia villiersi was described on the female sex from Mt. Nimba and Nion (Guinea) by Chopard (1954), who wrote that it is very similar to P. karschi, and differs mainly in the shape of tegmina, which are larger and more oval (length/width tegmina: 2.7). Specimens above listed from Côte d'Ivoire and Gabon have tegmina fairly oval, with the ratio length/width tegmina: 2.6; only Plangia ovalifolia (Bolívar, 1912) from Seychelles (Indian Ocean), described on the male sex, has such oval tegmina (length/width tegmina: 2.9). Thus, in the absence of males of $P$. villiersi, it is impossible to establish if $P$. ovalifolia and $P$. villiersi are conspecific, even if it seems unlikely that $P$. villiersi from West Africa and $P$. ovalifolia from Seychelles, remote islands about 7,000 km away from West Africa, belong to the same taxon.

\section{Plangia nebulosa Karsch, 1890}

Material examined. Cameroon, Barombi Station ( 9 holotype) (MfN); Liberia, Lofa County, Wologizi Mts., Ridge Camp 24-29.XI.2017 (Cold Cathode Light Trap), M. Aristophanous, S. Safian, G. Simonics, L. Smith (2o); Liberia, Sinoe County, Krahn-Bassa Reserve, Juboe River, 7.5 km SW Pellokon town, 14-21.I.2018 (MV Light Trap), M. Geiser, S. Safian, G. Simonics (1 +); Côte d'Ivoire, Yéalé Vill., Mt. Nimba, (380m) (light trap) 18-29.IV.2016, M. Aristophanous, M. Geiser, P. Moretto (3 3̂); Côte d'Ivoire, Yéalé Vill., Mt. Nimba (380m) (light trap) 8.V.2016, M. Aristophanous, M. Geiser, P. Moretto (1ठ); Côte d'Ivoire, Mt. Tonkoui Peak (1171m) 9-16.IV.2016 (light Trap), M. Aristophanous, P. Moretto (3ठ̂); Côte d'Ivoire, Taï National Park, Res. Station, 22.III-4.IV.2017 (UV), A. Aristophanous, M. Aristophanous, M. Geiser, P. Moretto (7우) (ANHRT); Côte d'Ivoire, Taï National Park, Res. Station, 22.III-4.IV.2017 (UV), P. Moretto (4+); Côte d'Ivoire, Comoe, Zamou (245m) 5-6.VI.2015 (UV), P. Moretto 
(2ð)); Côte d'Ivoire, Mt. Tonkoui (1171m) 18-20.VI.2015 (UV), P. Moretto (4仓); same data, 12-16.III.2017 (UV), P. Moretto (3ð); same data, 21.III.2017 (UV), P. Moretto (1ठ); same data, 23.IV.2017 (UV), P. Moretto (1ठ); Togo Kpalimé, Ft. Missahohe (610m) 29-30.VII.2013 (UV), P. Moretto (1ठ); Central African Republic, Sangha Reserve, lac 1, 24-25.XI.2010 (UV), P. Annoyer (19); Central African Republic, Angha, Mbaeré, Lac. 1, 1011.II.2012 (UV), P. Annoyer (1ㅇ) (BMPC).

Remarks. Plangia nebulosa was described by Karsch (1890) on the female sex, whose main character is the colour of tegmina with vague dark spots. The subgenital plate of the holotype is apically rounded. Later Griffini (1908) reported another female and described the unknown male from Mukonje Farm (Cameroon), with similar tegmina colours. Most specimens above listed show some vague spots on tegmina, males are lacking the black spot on the stridulatory area and have cerci incurved and black tipped, less stout than those of $P$. karschi. The stridulatory file consists of about 90 regularly spaced teeth.

Distribution. P. nebulosa should cover other than Cameroon, also Central African Republic, Togo, Côte d'Ivoire and Liberia. It was listed as Plangia sp. 2 by Massa et al. (2020).

\section{Plangia deminuta Griffini, 1908}

Material examined. Côte d'Ivoire, Taï Nat. Park, Res. Station 5-10.VII.2015 (light) (1 q) (NHM); Côte d'Ivoire, Taï Nat. Park, Res. Station 22.III-4.IV.2017 (light) (4ð̋, 1ㅇ) (BMPC); Côte d'Ivoire, Taï National Park, Res. Station (174m) (MV Light Trap) 25.III-17.IV.2017, A. Aristophanous, M. Aristophanous, M. Geiser, P. Moretto (2ð)) (ANHRT).

Distribution. Described from Cameroon (Griffini 1908), recorded from Central African Republic (Massa et al. 2020), here reported from Côte d'Ivoire.

\section{Eurycoplangiodes sanghaensis Massa, 2020}

Material examined. Côte d'Ivoire, Taï National Park, 25.III.2017 (light), P. Annoyer (1ठْ); Côte d'Ivoire, Mt. Tonkoui 12-16.III.2017, 1.IV.2017 (light), P. Moretto (20̂) (BMPC).

Distribution. Previously known from Central African Republic (Massa et al. 2020), now recorded also from Côte d'Ivoire.

\section{Oxygonatium huxleyi Ragge, 1980}

Material examined. Liberia, Lofa County, Foya Proposed Protected Area (530m) 10-19.XI.2017 (MV Light Trap), M. Aristophanous, S. Safian, G. Simonics, L. Smith (1ð) (ANHRT).

Distribution. Previously known from Ghana, Côte d'Ivoire, Central African Republic and Cameroon (Massa 2021a); it is here newly recorded from Liberia.

\section{Tribe Catoptropterigini Massa, 2016}

\section{Catoptropteryx lineata n. sp. (Figs. 34a-f)}

Material examined. Liberia, Sinoe County, Krahn-Bassa Reserve, Juboe River, 7.5 km SW Pellokon town, 14-



Description. Male. Colour. Green, abdomen and legs brown; dorsal margins of pronotum black and yellow, tegmina with a deep black line that covers all its posterior margin (Fig. 34a); cerci orange-blackish (Fig. 34b). Head and antennae. Eyes moderately large, globose, prominent. Fastigium of vertex narrower than scapus, not contiguous with the fastigium of frons. Face smooth. Thorax and legs. Anterior margin of pronotum just incurved, posterior margin rounded. Fore coxae armed. Fore femora unarmed, fore tibiae with open tympana, sulcate above, with 3 
outer and inner ventral spines +1 apical spur on each side, and 1 outer dorsal spur. Mid femora with 2 small spines on outer ventral margin, mid tibiae with 8 outer and inner ventral spines +1 apical spur on each side and 1 inner dorsal spur. Hind femora with 7 spines on outer and inner ventral margins, hind tibiae with many outer and inner dorsal and ventral spines +3 apical spurs on each side. Wings. $\mathrm{R}_{1}$ with 2 branches, Rs bifurcate, archedictyon denser and with smaller cells along centre of each area, MA unbranched. Tegmina 5.6 times longer than wide, with fore and hind margins more or less parallel, the fore margin only apically rounded. Stridulatory area of the left tegmen just raised, mirror on the right tegmen oval (Fig. 34a), stridulatory file under the left tegmen arched and consisting of ca. 40 regularly spaced teeth (Fig. 34d). Abdomen. Cerci decreasing in size, incurved and pointed (Fig. 34c); subgenital plate long and narrow, styli absent (Fig. 34b).

Measurements (mm). Male. Body length: 16.4; pronotum length: 4.1; pronotum height: 3.7 ; length hind femur: 19.0; tegmina: 26.5; tegmina width: 4.7.

Etymology. After Latin lineatus (= striped).

Affinities. Catoptropteryx lineata n. sp. could be misidentified as an atypical C. punctulata Karsch, 1890 without the characteristic black spots on the tegmina, but between the two species there are other differences, namely the shape of male cerci (compare Figs. $34 \mathrm{~b}$ and 34e) and the stridulatory file which in C. punctulata has very few acuminate teeth (compare Figs. 34d and 34f). The key to the species by Huxley (1970) should be modified before the dichotomy ' 11 ' as follows:

11a. Cerci of $\widehat{\partial}$ sinuose viewed in plane of principale curvature. 11

- Cerci of $\hat{o}$ not sinuose viewed in plane of principal curvature, only incurved. Catoptropteryx lineata $\mathrm{n}$. sp.

Distribution. Presently $C$. lineata $n$. sp. is known only from Liberia.

\section{Catoptropteryx ambigua Huxley, 1970}

Material examined. Liberia, Lofa County, Foya Proposed Protected Area (530m) 10-19.XI.2017 (MV Light Trap), M. Aristophanous, S. Safian, G. Simonics, L. Smith (1へ); Liberia, Lofa County, Wologizi Mts., Base Camp Forest (611m) 20.XI-1.XII.2017 (MV Light Trap), M. Aristophanous, S. Safian, G. Simonics, L. Smith (1ð); Liberia, Sinoe County, Krahn-Bassa Reserve, Juboe River, 7.5 km SW Pellokon town, 14-21.I.2018 (MV Light Trap), M. Geiser, S. Safian, G. Simonics (8ภ, 8 8 ); Liberia, Sinoe County, Krahn-Bassa Reserve, Juboe River, 7.5 km SW Pellokon town, 14-20.I.2018 (Blended Bulb 250w Light Trap), M. Geiser, S. Safian, G. Simonics (7ð̂, 1 1 ); Liberia, Sinoe County, 6.5 km NW Jacksonville, Forest near Solve Problem Vill. (103m) 23-27.I.2018 (MV Light Trap), M. Geiser, S. Safian, G. Simonics $(3 \hat{\jmath}, 2$, $)$ (ANHRT).

Distribution. It is known from Uganda (type locality), Cameroon, Democratic Republic of Congo, Central African Republic, and Gabon (Massa 2021a); it is new for Liberia.

\section{Catoptropteryx guttatipes Karsch, 1890}

Material examined. Sierra Leone, Tiwai Is., Moa River (120m) (light trap) 17-22.VI.2016, H. Takano, W. Miles,


M. Aristophanous, S. Safian, G. Simonics, L. Smith $(3 \hat{\jmath}, 3$ + $)$; Liberia, Lofa County, Wologizi Mts., Base Camp Forest (611m) 20.XI-1.XII.2017 (MV Light Trap), M. Aristophanous, S. Safian, G. Simonics, L. Smith (2ð, 4); Liberia, Sinoe County, Krahn-Bassa Reserve, Juboe River, 7.5 km SW Pellokon town, 14-21.I.2018 (MV Light Trap), M. Geiser, S. Safian, G. Simonics (1 $\jmath^{1}, 3$ \&); Liberia, Sinoe County, Krahn-Bassa Reserve, Juboe River, 7.5 km SW Pellokon town, 14-21.I.2018 (Cold Cathode UV Bucket Light), M. Geiser, S. Safian, G. Simonics (3ㅇ); Liberia, Sinoe County, Krahn-Bassa Reserve, Juboe River, 7.5 km SW Pellokon town, 14-20.I.2018 (Blended Bulb 250w Light Trap), M. Geiser, S. Safian, G. Simonics (3 9 ); Liberia, Sinoe County, 6.5 km NW Jacksonville, Forest near Solve Problem Vill. (103m) 23-27.I.2018 (MV Light Trap), M. Geiser, S. Safian, G. Simonics (1ð); Liberia, Lofa County, Wologizi Mts., Ridge Camp 2 (883m) (Cold Cathode UV Trap), 22-31.XI.2018, S. Safian, G. Simonics (1 $\overbrace{}^{\Uparrow}$ ); Togo, Fazao-Malfakassa NP, Mare aux crocodiles campsite (Sudanian Savannah/dry Forest) (505m)

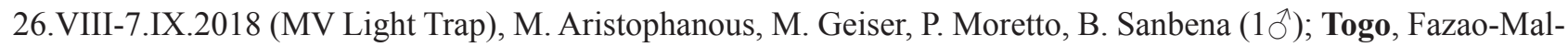
fakassa NP, Mare aux crocodiles campsite next to lake (Sudanian Savannah) (505m) 5-7.IX.2018 (Lepiled Light Trap), M. Aristophanous, M. Geiser, P. Moretto, B. Sanbena (1 +) (ANHRT). 
Distribution. Known from Cameroon (type locality), Uganda, Central African Republic, Gabon, Nigeria, and Equatorial Guinea (Massa 2021a); it is newly recorded from Sierra Leone, Liberia and Togo.

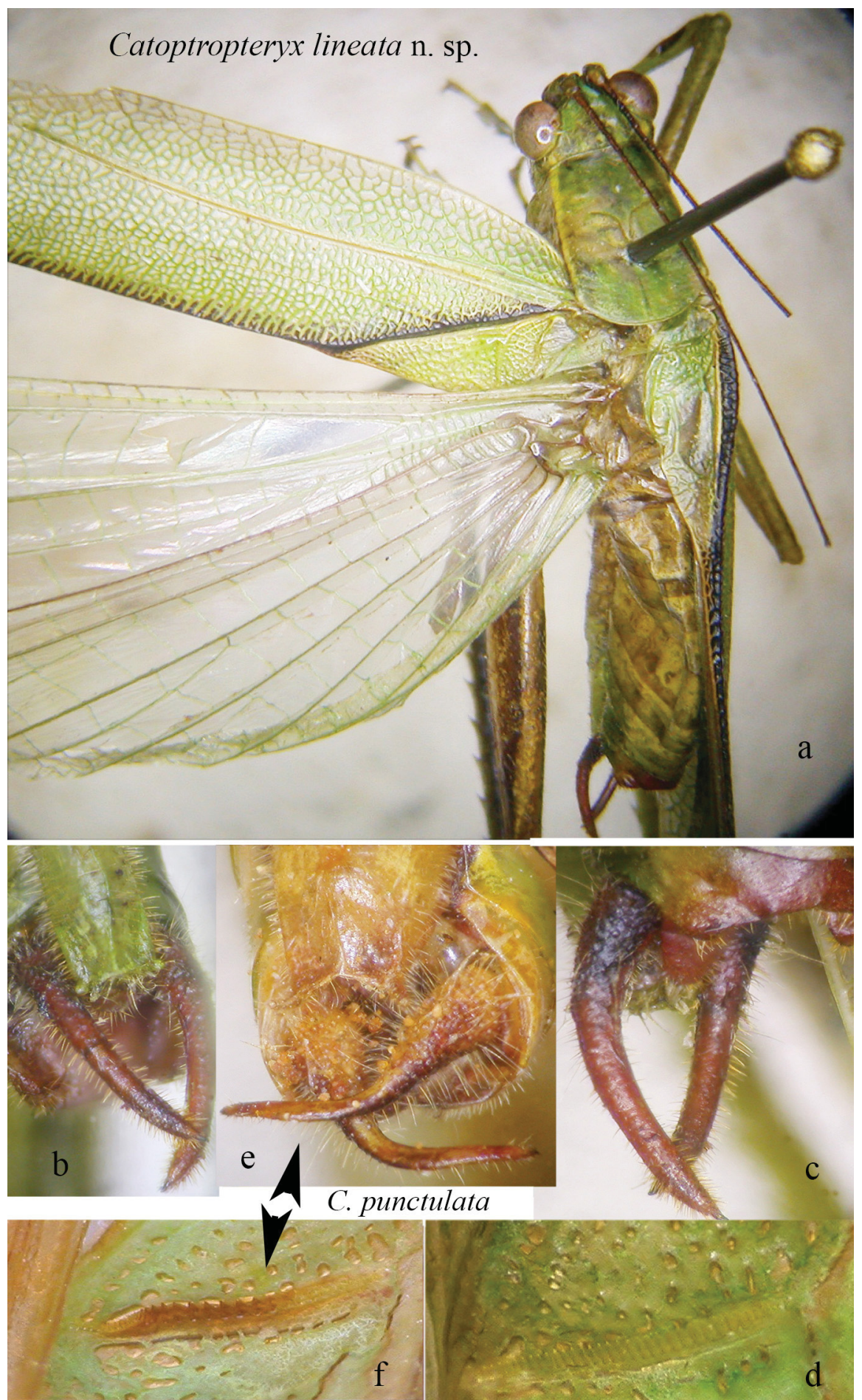

FIG. 34. Catoptropteryx lineata $\mathbf{n}$. sp.: a) dorsal view of the male holotype; b) ventral view of the male subgenital plate and cerci; c) dorsal view of male cerci; d) stridulatory file under the male left tegmen; Catoptropteryx punctulata Karsch, 1890: e) ventral view of male subgenital plate and cerci; f) stridulatory file under the male left tegmen.

\section{Catoptropteryx nana Huxley, 1970}

Material examined. Liberia, Sinoe County, Krahn-Bassa Reserve, Juboe River, 7.5 km SW Pellokon town, 1420.I.2018 (Blended Bulb 250w Light Trap), M. Geiser, S. Safian, G. Simonics (1ठ) (ANHRT). 
Distribution. It is known from Cameroon (type locality), Central African Republic, and Gabon (Massa 2021a); it is newly recorded from Liberia.

\section{Tribe Holochlorini Brunner von Wattenwyl, 1878}

\section{Arantia (Arantia) angustipennis Chopard, 1954}

Material examined. Côte d'Ivoire, Man, Mt. Tonkoui (1200m) 6.IV.2017 (light) (1仓̂) (BMPC); Côte d'Ivoire, Mt. Tonkoui Peak (1171m) 20-27.V.2018 (MV Light Trap), M. Aristophanous, W. Miles, P. Moretto, Y. Outtara (1ठ) (ANHRT).

Distribution. Previously known from Guinea and Ghana (Hemp \& Massa 2017), now recorded also from Côte d'Ivoire.

\section{Arantia (Arantia) ivoriana Hemp \& Massa, 2017}

Material examined. Côte d'Ivoire, Abidjan, Banco Forest (Banco NP) (39-48m) (gen. coll.) 21.IV-1.V.2017, A. Aristophanous, M. Aristophanous, M. Geiser, P. Moretto (1ठ) (ANHRT).

Distribution. Previously known from the holotype (collected in the Taï National Park, Côte d'Ivoire) (Hemp \& Massa 2017), now recorded from the Banco National Park, Côte d'Ivoire.

\section{Arantia (Arantia) manca Bolívar, 1906}

Material examined. Côte d'Ivoire, Abidjan, Banco Forest (Banco PN) (39-48m) 5²3'03.8'N, 403'11.2”W (gen. coll.) 21.IV-1.V.2017, A. Aristophanous, M. Aristophanous, M. Geiser, P. Moretto (ANHRT).

Distribution. Known from Cameroon, Democratic Republic of Congo, Angola, Ghana, and Central African Republic (Massa et al. 2020), here recorded also from Côte d'Ivoire.

\section{Arantia (Arantia) marginata Massa, 2021 (Figs. 35-38, 39a-e)}

Material examined. Côte d'Ivoire, Mt. Tonkoui Peak (1171m) 9-16.IV.2016 (light Trap), M. Aristophanous, P. Moretto (21ðึ); Côte d'Ivoire, Yéalé Vill., Mt. Nimba, (380m) (light trap) 18-29.IV.2016, M. Aristophanous, M. Geiser, P. Moretto (1 $\left.\delta^{\Uparrow}\right)$; Côte d'Ivoire, Mt. Nimba camp (823m) (light trap) 28.IV-8.V.2016, M. Aristophanous, M. Geiser, P. Moretto (1ठ); Côte d'Ivoire, Bossématié For. Classée (180m) (light trap) 25-31.VII.2016, M. Aristophanous, P. Moretto (2ð̂); Côte d'Ivoire, Comoe, Kromambira Vill. (240m) (gen. coll.) 3-13.VIII.2016, M. Aristophanous, P. Moretto (1ठ̋); Côte d'Ivoire, Comoe, Kromambira Vill. (240m) (Light Trap) 3-13.VIII.2016, M. Aristophanous, P. Moretto (1ठ̋); Côte d'Ivoire, Taï National Park, Res. Station (174m) (MV Light Trap) 25.III-17.IV.2017, A. Aristophanous, M. Aristophanous, M. Geiser, P. Moretto (5ð); Côte d'Ivoire, Taï National Park, Res. Station (174m) (Canopy Light Trap, 40m ab. ground) 27-31.III.2017, A. Aristophanous, M. Aristophanous, M. Geiser, P. Moretto (2ð̋); Côte d'Ivoire, Mt. Tonkoui Peak (1171m) 20-27.V.2018 (MV Light Trap), M. Aristophanous, W. Miles, P. Moretto, Y. Outtara (1ठ); Côte d'Ivoire, Denguele Classified Forest (sudanian forest) (479m) (MV Light Trap), 6-14.VI.2018, M. Aristophanous, W. Miles, P. Moretto, Y. Outtara (1 đ̂); Côte d'Ivoire, Mt. Tonkoui Peak (1171m) 19-27.XI.2019 (MV light Trap), M. Aristophanous, V. Dérozier, P. Moretto, S. Ouattara (1ठ); Liberia, Lofa County, Foya Proposed Protected Area (530m) 10-19.XI.2017 (MV Light Trap), M. Aristophanous, S. Safian, G. Simonics, L. Smith (13ð̋); Liberia, Lofa County, Wologizi Mts., Base Camp Forest (611m) 20.XI-1.XII.2017 (MV Light Trap), M. Aristophanous, S. Safian, G. Simonics, L. Smith (2ð̋); Liberia, Sinoe County, Krahn-Bassa Reserve, Juboe River, 7.5 km SW Pellokon town, 14-21.I.2018 (MV Light Trap), M. Geiser, S. Safian, G. Simonics (1§ึ); Liberia, Sinoe County, Krahn-Bassa Reserve, Juboe River, 7.5 km SW Pellokon town, 14-20.I.2018 (Blended 

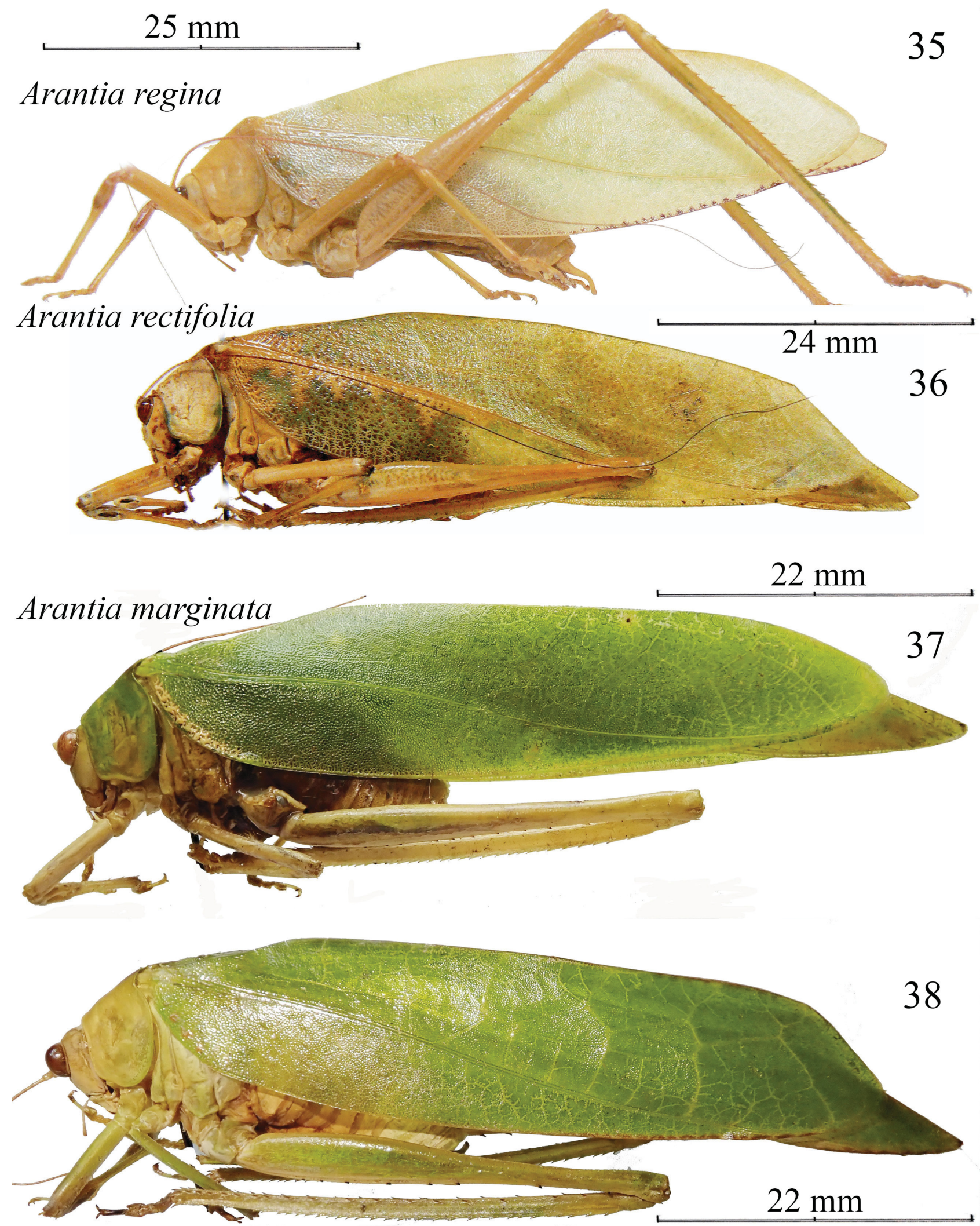

FIGS. 35-38. Comparison of some Arantia with cerci long and slightly sinuous with a black, chitinous and rounded apex: Arantia (Euarantia) regina Karsch, 1889 (35), Arantia (Euarantia) rectifolia Brunner von Wattenwyl, 1878 (36), and Arantia (Arantia) marginata Massa, 2021 (two specimens: 37 and 38). 


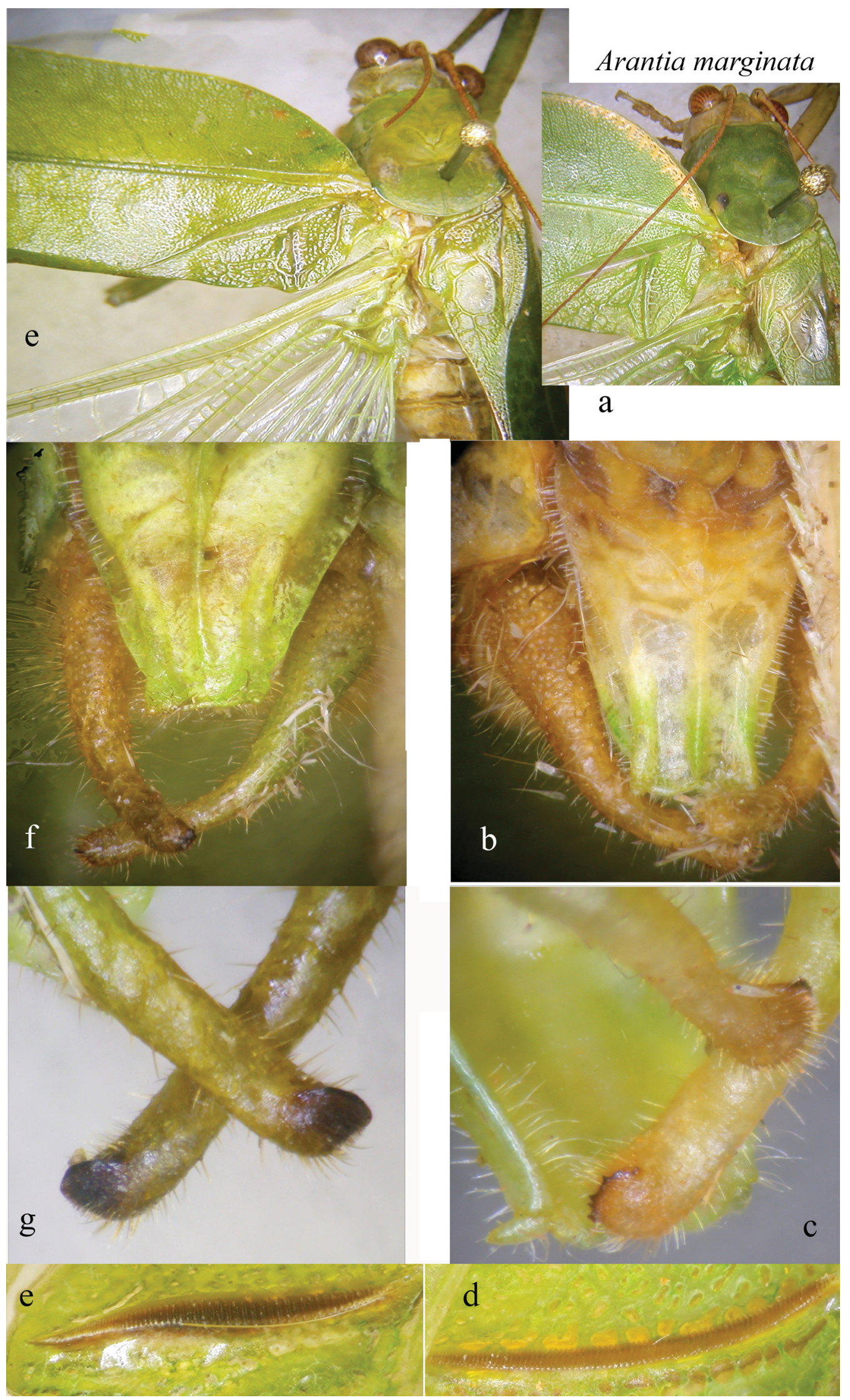

FIG. 39. Arantia (Arantia) marginata Massa, 2021: a) dorsal view of male head, pronotum and tegmina; b) ventral view of the male subgenital plate and cerci; c) male cerci apex; d) stridulatory file under the male left tegmen; Arantia (Arantia) aff. marginata: e) dorsal view of male head, pronotum and tegmina; f) ventral view of the male subgenital plate and cerci; g) male cerci apex; e) stridulatory file under the male left tegmen. 
Bulb 250w Light Trap), M. Geiser, S. Safian, G. Simonics (1 $\overbrace{}^{\Uparrow})$; Liberia, Sinoe County, 6.5 km NW Jacksonville,

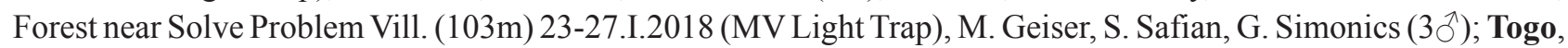

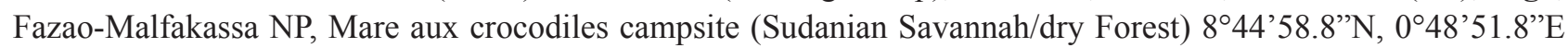
(505m) 26.VIII-7.IX.2018 (MV Light Trap), M. Aristophanous, M. Geiser, P. Moretto, B. Sanbena (3ð); Guinea, Ditinn, Chute de Ditinn (771m) (MV Light Trap) 18-25.IX.2019, M. Geiser, M. Leno, S. Koivagui, W. Miles, L. Mulvaney, S. Safian (1今゙); Guinea, Ditinn, Chute de Ditinn (771m) (Lepiled Light Trap) 18-25.IX.2019, M. Geiser, M. Leno, S. Koivagui, W. Miles, L. Mulvaney, S. Safian (1 $\overbrace{}^{\Uparrow})$ (ANHRT); Côte d'Ivoire, Mt. Tonkoui (1171m) X.2016 (light), P. Moretto (3ð)); same data, 22.IV.2017 (light), P. Moretto (1ð); same data, 4.VI.2018 (light), P. Moretto (1ð); Côte d'Ivoire, Taï National Park, Res. Station, 16-20.III.2017 (UV), B. Massa (3ð); same data, 22.III-4.IV.2017 (UV), P. Moretto, P. Annoyer (3ठ)); Côte d'Ivoire, Zamou, Bandoukou VII.2004, P. Moretto (3ð); Côte d'Ivoire, Zamou, Kolomabira VII.2015 (light), P. Moretto (1ठ̂); Central African Republic, Sangha-Ndoki Nat. Park, Camp 2, 21-23.X.2008 (UV), P. Annoyer (3ð); same data, Lac 1, 28-29.II.2012 (UV), P. Moretto (1§) (BMPC).

Taxonomical remarks. Following Heller et al. (2014), Hemp \& Massa (2017) included the group of Arantia regina-rectifolia-tanzanica into the subgenus Euarantia, having the width of the tegmina greater than $12 \mathrm{~mm}$ and the hind tibiae curved, but they highlighted that $A$. rectifolia Brunner von Wattenwyl, 1878 may be intermediate between Arantia and Euarantia, because in some specimens examined the tegmina width ranged between 12.0 to 13.8 $\mathrm{mm}$, and the hind tibiae were not as curved as it was given by Heller et al. (2014) for Euarantia (see Figs. 35-38). This group of species are characterized by faintly visible 4-5 ivory or more tawny to orange spots on anterior margin of tegmina, and cerci long and slightly sinuous with a black, chitinous and rounded apex. When Massa (2021a) described A. marginata from Gabon, he wrote that it is characterized by tegmina narrower than $12 \mathrm{~mm}$, cerci long and in-curved, and just sinuous with a black, chitinous and rounded apex with 4-5 tiny spines, and stridulatory file arcuate, narrow, consisting of ca. 65 evenly spaced teeth. Really, A. marginata is very similar to A. rectifolia and only a careful observation may separate them. In the material above listed a huge number of specimens at the beginning considered A. rectifolia is included. They were collected in Central African Republic, Côte d'Ivoire, Liberia, Guinea, and their tegmina width resulted narrower than $12 \mathrm{~mm}$; some of them were also mounted with the left tegmen open and it was possible to check the stridulatory file. Interestingly, most of them have the stridulatory file of ca. 65 teeth (as in A. marginata; in A. rectifolia they are 45-50, not 80-85 as reported by Hemp \& Massa 2017), but in at least three specimens (from Liberia, with tegmina width 10.5-11.0 mm) it was different, shorter and with a fewer number of teeth (Figs. 39d and 39e). In all the specimens the hind tibiae are inappreciably curved. Cerci of these specimens resulted to be a little different (compare Figs. 39b and 39c with Figs. 39f and 39g), but observing some specimens of $A$. marginata with 65 teeth in the stridulatory file, they may possess cerci similar to those in Fig. $39 \mathrm{~g}$ (incidentally very similar to those of $A$. rectifolia). It seems very likely that there are at least two species very similar within the series of specimens identified as A. marginata, but it is not possible to find a character (other than the stridulatory file) allowing a sure separation (see Figs. 39a-e). The inclusion of the above group of species in different subgenera is hardly justified.

\section{Arantia (Arantia) quinquemaculata Hemp et Massa, 2017}

Material examined. Liberia, Lofa County, Foya Proposed Protected Area (530m) 10-19.XI.2017 (MV Light Trap), M. Aristophanous, S. Safian, G. Simonics, L. Smith (12§); Liberia, Lofa County, Wologizi Mts., Base Camp Forest (611m) 20.XI-1.XII.2017 (MV Light Trap), M. Aristophanous, S. Safian, G. Simonics, L. Smith (3ठ̂); Liberia, Sinoe County, Krahn-Bassa Reserve, Juboe River, 7.5 km SW Pellokon town, 14-20.I.2018 (Blended Bulb 250w Light Trap), M. Geiser, S. Safian, G. Simonics (2今) (ANHRT).

Distribution. Known from Central African Republic, Côte d'Ivoire, Gabon and Togo (Hemp \& Massa 2017), newly recorded from Liberia.

\section{Arantia (Arantia) brevipes Chopard, 1954}

Material examined. Liberia, Lofa County, Wologizi Mts., Base Camp Forest (611m) 20.XI-1.XII.2017 (MV Light Trap), M. Aristophanous, S. Safian, G. Simonics, L. Smith (1ð̋); Liberia, Lofa County, Wologizi Mts., Ridge Camp 24-29.XI.2017 (Cold Cathode Light Trap), M. Aristophanous, S. Safian, G. Simonics, L. Smith (1 $\overbrace{}^{\text {) }}$ (ANHRT). 
Distribution. Previously known from Guinea, Sierra Leone, Ghana and Côte d'Ivoire (Hemp \& Massa 2017), now recorded from Liberia.

\section{Arantia (Euarantia) regina Karsch, 1889}

Material examined. Sierra Leone, Loma Mts., Closed-canopy for. (1050m) (light trap) 7-10.VI.2016, H. Takano, W. Miles, R. Goff (2 +); Liberia, Lofa County, Foya Proposed Protected Area (530m) 10-19.XI.2017 (MV Light Trap), M. Aristophanous, S. Safian, G. Simonics, L. Smith (14^, 2 \%); Liberia, Lofa County, Wologizi Mts., Base Camp Forest (611m) 20.XI-1.XII.2017 (MV Light Trap), M. Aristophanous, S. Safian, G. Simonics, L. Smith (50̂, 19); Liberia, Sinoe County, Krahn-Bassa Reserve, Juboe River, 7.5 km SW Pellokon town, 14-21.I.2018 (MV Light Trap), M. Geiser, S. Safian, G. Simonics $(6 \hat{\jmath}, 5 q)$; Liberia, Sinoe County, Krahn-Bassa Reserve, Juboe River, 7.5 km SW Pellokon town, 14-20.I.2018 (Blended Bulb 250w Light Trap), M. Geiser, S. Safian, G. Simonics (2ð)); Liberia, Sinoe County, 6.5 km NW Jacksonville, Forest near Solve Problem Vill. (103m) 23-27.I.2018 (MV Light Trap), M. Geiser, S. Safian, G. Simonics (2ð) (ANHRT).

Distribution. Previously known from Gabon, Cameroon, Equatorial Guinea, Central African Republic, Democratic Republic Congo, Togo, Burkina Faso, Côte d'Ivoire (Hemp \& Massa 2017), unrecorded from Sierra Leone and Liberia.

\section{Arantia (Euarantia) tibiaspinosa Hemp et Massa, 2017}

Material examined. Liberia, Sinoe County, Krahn-Bassa Reserve, Juboe River, $7.5 \mathrm{~km}$ SW Pellokon town, 14-

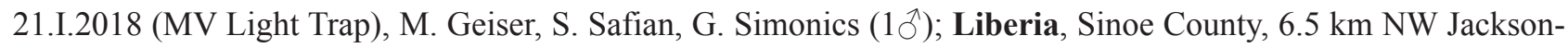
ville, Forest near Solve Problem Vill. (103m) 23-27.I.2018 (MV Light Trap), M. Geiser, S. Safian, G. Simonics (2ð) (ANHRT).

Distribution. Previously known from Côte d'Ivoire (Hemp \& Massa 2017), now recorded also from Liberia.

\section{Arantia (Euarantia) retinervis Karsch, 1889}

Material examined. Liberia, Lofa County, Foya Proposed Protected Area (530m) 10-19.XI.2017 (MV Light Trap), M. Aristophanous, S. Safian, G. Simonics, L. Smith (1ð); Liberia, Sinoe County, 6.5 km NW Jacksonville, Forest near Solve Problem Vill. (103m) 23-27.I.2018 (MV Light Trap), M. Geiser, S. Safian, G. Simonics (2ð); Sierra Leone, Tiwai Is., Moa River (120m) (light trap) 17-22.VI.2016, H. Takano, W. Miles, R. Goff (1 $\overbrace{}^{\Uparrow})$ (ANHRT).

Distribution. Previously known from Sierra Leone, Guinea, Togo, Côte d'Ivoire, Equatorial Guinea, Central African Republic, Democratic Republic of Congo, Cameroon and Gabon (Hemp \& Massa 2017, Massa 2021a), now recorded also from Liberia.

\section{Arantia (Euarantia) scurra Karsch, 1896}

Material examined. Liberia, Lofa County, Foya Proposed Protected Area (530m) 10-19.XI.2017 (MV Light Trap), M. Aristophanous, S. Safian, G. Simonics, L. Smith (6ð); Liberia, Sinoe County, Krahn-Bassa Reserve, Juboe River, 7.5 km SW Pellokon town, 14-21.I.2018 (MV Light Trap), M. Geiser, S. Safian, G. Simonics (1 ) (ANHRT).

Distribution. Previously known from Cameroon and Central African Republic (Hemp \& Massa 2017), here recorded also from Liberia.

\section{Arantia (Goetia) galbana (Karsch, 1891) (Fig. 30)}

Material examined. Liberia, Lofa County, Foya Proposed Protected Area (530m) 10-19.XI.2017 (MV Light Trap), M. Aristophanous, S. Safian, G. Simonics, L. Smith (2ð); Liberia, Lofa County, Wologizi Mts., Base Camp Forest 
(611m) 20.XI-1.XII.2017 (MV Light Trap), M. Aristophanous, S. Safian, G. Simonics, L. Smith (1ðð, 19); Liberia, Sinoe County, 6.5 km NW Jacksonville, Forest near Solve Problem Vill. (103m) 23-27.I.2018 (MV Light Trap), M. Geiser, S. Safian, G. Simonics (1 9 ) (ANHRT).

Distribution. Previously known from Cameroon, Equatorial Guinea, Ghana and Côte d'Ivoire (Hemp \& Massa 2017), here recorded also from Liberia.

\section{Dapanera irregularis Karsch, 1890}

Material examined. Liberia, Lofa County, Foya Proposed Protected Area (530m) 10-19.XI.2017 (MV Light Trap), M. Aristophanous, S. Safian, G. Simonics, L. Smith (2犬) ; Liberia, Lofa County, Wologizi Mts., Base Camp Forest (611m) 20.XI-1.XII.2017 (MV Light Trap), M. Aristophanous, S. Safian, G. Simonics, L. Smith (20̂); Liberia, Sinoe County, Krahn-Bassa Reserve, Juboe River, 7.5 km SW Pellokon town, 14-20.I.2018 (Blended Bulb 250w Light Trap), M. Geiser, S. Safian, G. Simonics (1 $\overbrace{}^{\Uparrow})$ (ANHRT).

Distribution. Previously known from Democratic Republic of Congo, Central African Republic, Gabon, Cameroon, Côte d'Ivoire and Ghana (Massa 2021a), here recorded also from Liberia.

\section{Dapanera brevistylata Massa, 2020}

Material examined. Liberia, Lofa County, Foya Proposed Protected Area (530m) 10-19.XI.2017 (MV Light Trap), M. Aristophanous, S. Safian, G. Simonics, L. Smith (1ð); Gabon, Mikongo (Rougier), Mts de Cristal (secondary forest) (430m) 28.VII-12.VIII.2019 (MV Light Trap) (1ठ) (ANHRT).

Distribution. Previously known from Central African Republic (Massa et al. 2020), here recorded also from Gabon and Liberia.

\section{Tribe Plangiopsidini Cadena-Castañeda, 2015}

\section{Plangiopsis semiconchata Karsch, 1889}

Material examined. Liberia, Sinoe County, Krahn-Bassa Reserve, Juboe River, $7.5 \mathrm{~km}$ SW Pellokon town, 1421.I.2018 (MV Light Trap), M. Geiser, S. Safian, G. Simonics (1 ○̋); Liberia, Sinoe County, Krahn-Bassa Reserve, Juboe River, 7.5 km SW Pellokon town, 14-20.I.2018 (Blended Bulb 250w Light Trap), M. Geiser, S. Safian, G. Simonics (3へ̂); Liberia, Sinoe County, 6.5 km NW Jacksonville, Forest near Solve Problem Vill. (103m) 2327.I.2018 (MV Light Trap), M. Geiser, S. Safian, G. Simonics (4^) (ANHRT).

Distribution. Previously known from Cameroon, Côte d'Ivoire, Ghana, Central African Republic and Gabon (Massa 2021a), here recorded also from Liberia.

\section{Plangiopsis foraminata Karsch, 1891}

Material examined. Liberia, Sinoe County, Krahn-Bassa Reserve, Juboe River, $7.5 \mathrm{~km}$ SW Pellokon town, 1421.I.2018 (MV Light Trap), M. Geiser, S. Safian, G. Simonics (1ðð, 1ㅇ) (ANHRT).

Distribution. Previously known from Ghana, Central African Republic and Gabon, here recorded also from Liberia.

Tribe Otiaphysini Karsch, 1889

Tetraconcha aristophanousi Massa, 2017

Material examined. Liberia, Lofa County, Foya Proposed Protected Area (530m) 10-19.XI.2017 (MV Light Trap), 
M. Aristophanous, S. Safian, G. Simonics, L. Smith (7ठ̋); Liberia, Lofa County, Foya Proposed Protected Area (530m) 10-19.XI.2017 (8w Actinic Light Trap Bucket), M. Aristophanous, S. Safian, G. Simonics, L. Smith (1ठ); Liberia, Lofa County, Wologizi Mts., Base Camp Forest (611m) 20.XI-1.XII.2017 (MV Light Trap), M. Aristophanous, S. Safian, G. Simonics, L. Smith (16ð); Liberia, Lofa County, Wologizi Mts., Ridge Camp 24-29.XI.2017 (Cold Cathode Light Trap), M. Aristophanous, S. Safian, G. Simonics, L. Smith (1ठ); Liberia, Sinoe County, Krahn-Bassa Reserve, Juboe River, 7.5 km SW Pellokon town, 14-21.I.2018 (MV Light Trap), M. Geiser, S. Safian, G. Simonics (1ठ̂); Liberia, Sinoe County, 6.5 km NW Jacksonville, Forest near Solve Problem Vill. (103m) 2327.I.2018 (MV Light Trap), M. Geiser, S. Safian, G. Simonics (1 $\left.{ }_{3}^{3}\right)$; Guinea, Guinée forestière, Bossou For. \& Inst. Rech. Env. Bossou (Lowland Forest-Farmland) (690m) (Lepiled Light Trap) 24-31.VI.2019, V. Dérozier, J. Suha Dore, S. Koivagui, W. Miles, S. Safian, R. Warner (1 $\overbrace{}^{\Uparrow})$ (ANHRT).

Distribution. Previously known from Côte d'Ivoire and Sierra Leone (Massa 2017), now recorded also from Liberia and Guinea.

\section{Tetraconcha ruzzieri Massa, 2017}

Material examined. Liberia, Lofa County, Wologizi Mts., Base Camp Forest (611m) 20.XI-1.XII.2017 (MV Light Trap), M. Aristophanous, S. Safian, G. Simonics, L. Smith (1へ̋); Liberia, Sinoe County, 6.5 km NW Jacksonville, Forest near Solve Problem Vill. (103m) 23-27.I.2018 (MV Light Trap), M. Geiser, S. Safian, G. Simonics (1ठ) (ANHRT).

Distribution. Previously known only from Côte d'Ivoire (Massa 2017), now recorded also from Liberia.

\section{Tribe Phlaurocentrini Karsch, 1889}

\section{Leiodontocercus angustipennis Chopard, 1954 (Figs. 40a-b)}

Material examined. Côte d'Ivoire, Taï National Park, Res. Station (174m) (MV Light Trap) 25.III-17.IV.2017, A. Aristophanous, M. Aristophanous, M. Geiser, P. Moretto (1ð̄, 2 ㅇ); Liberia, Sinoe County, 6.5 km NW Jacksonville, Forest near Solve Problem Vill. (103m) 23-27.I.2018 (MV Light Trap), M. Geiser, S. Safian, G. Simonics (19) (ANHRT).

Remarks. The genus Leiodontocercus has been revised recently by Massa (2020), who examined only the holotype; now another male was available and it was possible to examine the stridulatory file, consisting of ca. 60 teeth (Fig. 40a), similar to that of L. philipporum Massa, 2020, but teeth are deeper; however, cerci of $L$. angustipennis are stout with an inner globose bulge (Fig. 40b)

Distribution. It is known from Sierra Leone and Guinea (Massa 2020), newly recorded from Côte d'Ivoire and Liberia.

\section{Tribe Preussiini Karsch, 1890 (Figs. 40c, 41-44)}

Remarks. When Ragge (1981) described Brycoptera lobata, he did not establish which tribe of Phaneropterinae it could belong to. Characters of this taxon are very peculiar and represent an extreme modification of the habitus towards a foliaceous aspect eroded by the possible mandibles of a caterpillar; all the legs are laterally expanded and this makes it objectively difficult to recognize the tribe it belongs to (Fig. 40c). Here it is proposed to ascribe this taxon to the tribe Preussiini Karsch, 1890, characterized as follows: foliaceous aspect, large size (body length: 22.0-26.0 mm), fastigium of vertex narrow, pointed and furrowed, as large as first antennal segment, face large and short, fastigium of frons with a small furrowed horn. Eyes oval, moderately prominent, scapus placed within an area with raised margins. Pronotum flat and smooth, nearly as long as high, with evident lateral margins and well-developed humeral excision, lateral lobes rounded on hind and lower margins. Legs short and stout; fore coxae armed, fore femora short and laterally flattened, fore tibiae dorsally sulcate, tympana conchate. Tegmina well developed, slightly shorter than hind wings. Tenth tergite unmodified, subgenital plate concave, long and narrow, provided with styli. Cerci stout and in-curved (Massa 2016). 


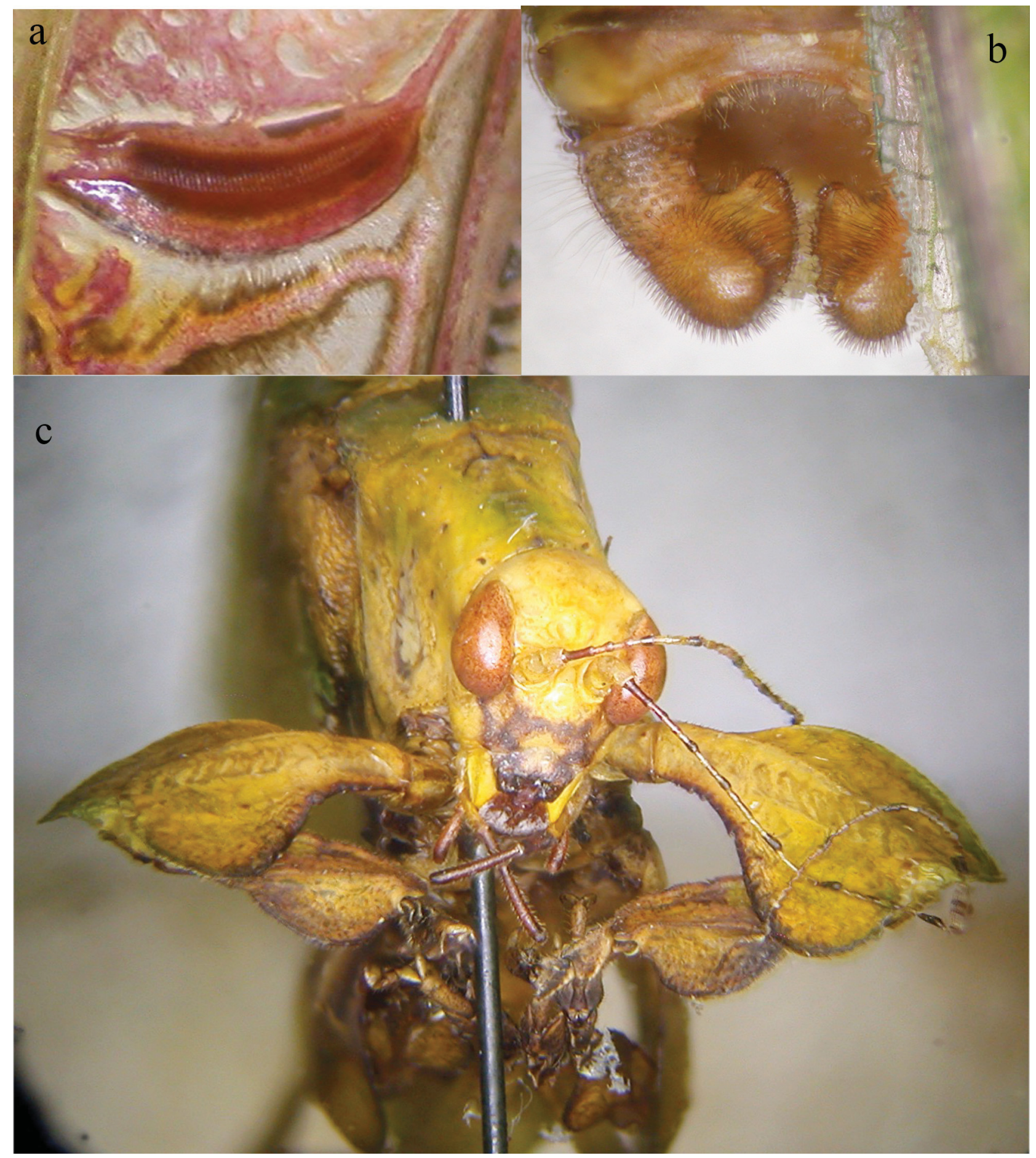

FIG. 40. Leiodontocercus angustipennis Chopard, 1954: a) stridulatory file under the male left tegmen; b) cerci of the male; Brycoptera lobata Ragge, 1981: frontal view of head and fore legs.

\section{Brycoptera lobata Ragge, 1981 (Fig. 40c, 44)}

Material examined. Côte d'Ivoire, Man, Mt. Tonkoui (1171m) 3.IV.2017 (light) (1ठ), 10.IV.2018 (light) (1ठ) (BMPC); Côte d'Ivoire, Mt. Tonkoui Peak 7²7’15.2”N, 7³8’12.5”W (1171m) 9-16.IV.2016 (light Trap), M. Aristophanous, P. Moretto (1 $\overbrace{}^{\Uparrow})$ (ANHRT).

Distribution. It is known from Angola, Cameroon, Central African Republic, Equatorial Guinea, Côte d'Ivoire and Uganda (Massa et al. 2020). 
Material examined. Liberia, Lofa County, Wologizi Mts., Base Camp Forest (611m) 20.XI-1.XII.2017 (MV Light Trap), M. Aristophanous, S. Safian, G. Simonics, L. Smith (1§̋); Liberia, Sinoe County, 6.5 km NW Jacksonville, Forest near Solve Problem Vill. (103m) 23-27.I.2018 (MV Light Trap), M. Geiser, S. Safian, G. Simonics (10̋); Liberia, Lofa County, Wologizi Mts., Elephant Ridge (1002m) (Cold Cathode UV Trap) 23-26.XI.2018, S. Safian, G. Simonics (1§); Guinea, Guinée forestière, Bossou For. \& Inst. Rech. Env. Bossou (Lowland Forest-Farmland) (690m) (8W UV Cold Cathode Light) 24-31.VI.2019, V. Dérozier, J. Suha Dore, S. Koivagui, W. Miles, S. Safian, R. Warner (1§) (ANHRT).

Distribution. Previously known from Cameroon, Central African Republic, Côte d'Ivoire, Ghana (Massa et al. 2020), here it is recorded also from Liberia and Guinea.

\section{Tribe Morgeniini Karsch, 1890}

\section{Morgenia rubricornis Sjöstedt, 1913}

Material examined. Liberia, Sinoe County, Krahn-Bassa Reserve, Juboe River, 7.5 km SW Pellokon town, 1421.I.2018 (MV Light Trap), M. Geiser, S. Safian, G. Simonics (1§ึ); Liberia, Sinoe County, 6.5 km NW Jacksonville, Forest near Solve Problem Vill. (103m) 23-27.I.2018 (MV Light Trap), M. Geiser, S. Safian, G. Simonics (2ð) (ANHRT).

Distribution. Previously known from Democratic Republic of Congo, Central African Republic, Gabon and Côte d'Ivoire (Massa et al. 2018), now recorded also from Liberia.

\section{Morgenia hamuligera Karsch, 1890}

Material examined. Liberia, Lofa County, Foya Proposed Protected Area (530m) 10-19.XI.2017 (MV Light Trap), M. Aristophanous, S. Safian, G. Simonics, L. Smith (5ð); Liberia, Lofa County, Wologizi Mts., Base Camp Forest (611m) 20.XI-1.XII.2017 (MV Light Trap), M. Aristophanous, S. Safian, G. Simonics, L. Smith (1ð); Liberia, Sinoe County, Krahn-Bassa Reserve, Juboe River, 7.5 km SW Pellokon town, 14-21.I.2018 (MV Light Trap), M. Geiser, S. Safian, G. Simonics (3§^); Liberia, Sinoe County, Krahn-Bassa Reserve, Juboe River, 7.5 km SW Pellokon town, 14-20.I.2018 (Blended Bulb 250w Light Trap), M. Geiser, S. Safian, G. Simonics (4ત̋); Liberia, Sinoe County, 6.5 km NW Jacksonville, Forest near Solve Problem Vill. (103m) 23-27.I.2018 (MV Light Trap), M. Geiser, S. Safian, G. Simonics (19); Togo, Fazao-Malfakassa NP, Mare aux crocodiles campsite (Sudanian Savannah/dry Forest) (505m) 26.VIII-7.IX.2018 (Actinic Light Trap), M. Aristophanous, M. Geiser, P. Moretto, B. Sanbena (1 $\left.{ }^{\lambda}\right)$ (ANHRT).

Distribution. Known from Cameroon, Equatorial Guinea, Democratic Republic of Congo, Central African Republic, Gabon and Côte d'Ivoire (Massa et al. 2018), now recorded also from Liberia and Togo.

\section{Morgenia melica Karsch, 1893}

Material examined. Sierra Leone, Tiwai Is., Moa River (120m) (light trap) 17-22.VI.2016, H. Takano, W. Miles, R. Goff (2ð); Liberia, Lofa County, Foya Proposed Protected Area (530m) 10-19.XI.2017 (MV Light Trap), M. Aristophanous, S. Safian, G. Simonics, L. Smith $(7 \hat{\partial}, 1$ 우); Liberia, Lofa County, Foya Proposed Protected Area (530m) 10-19.XI.2017 (8w Actinic Light Trap Bucket), M. Aristophanous, S. Safian, G. Simonics, L. Smith (3ð); Liberia, Sinoe County, Krahn-Bassa Reserve, Juboe River, 7.5 km SW Pellokon town, 14-21.I.2018 (MV Light Trap), M. Geiser, S. Safian, G. Simonics (7ð̂, 1 ); Liberia, Sinoe County, Krahn-Bassa Reserve, Juboe River, 7.5 km SW Pellokon town, 14-20.I.2018 (Blended Bulb 250w Light Trap), M. Geiser, S. Safian, G. Simonics (1ð^, 2 ㅇ); Liberia, Sinoe County, 6.5 km NW Jacksonville, Forest near Solve Problem Vill. (103m) 23-27.I.2018 (MV Light Trap), M. Geiser, S. Safian, G. Simonics (11ठ̋) (ANHRT). 
Distribution. Previously known from Cameroon, Democratic Republic of the Congo, Central African Republic, Uganda, Gabon and Côte d'Ivoire (Massa et al. 2018), now recorded also from Liberia.

\section{Morgenia modulata Karsch, 1896}

Material examined. Liberia, Lofa County, Foya Proposed Protected Area (530m) 10-19.XI.2017 (MV Light Trap), M. Aristophanous, S. Safian, G. Simonics, L. Smith (13ð); Liberia, Lofa County, Foya Proposed Protected Area (530m) 10-19.XI.2017 (8w Actinic Light Trap Bucket), M. Aristophanous, S. Safian, G. Simonics, L. Smith (1§)); Liberia, Lofa County, Wologizi Mts., Base Camp Forest (611m) 20.XI-1.XII.2017 (MV Light Trap), M. Aristophanous, S. Safian, G. Simonics, L. Smith (5犬̂, 2); Liberia, Sinoe County, Krahn-Bassa Reserve, Juboe River, 7.5 km SW Pellokon town, 14-20.I.2018 (Blended Bulb 250w Light Trap), M. Geiser, S. Safian, G. Simonics (1ð̊, 1 ) Liberia, Sinoe County, 6.5 km NW Jacksonville, Forest near Solve Problem Vill. (103m) 23-27.I.2018 (MV Light Trap), M. Geiser, S. Safian, G. Simonics (2+) (ANHRT).

Distribution. Previously known from Cameroon, Central Africa Republic, Gabon, Togo and Côte d'Ivoire (Massa et al. 2018), now recorded also from Liberia.

\section{Morgenia spathulifera Griffini, 1908}

Material examined. Liberia, Sinoe County, Krahn-Bassa Reserve, Juboe River, 7.5 km SW Pellokon town, 1421.I.2018 (MV Light Trap), M. Geiser, S. Safian, G. Simonics (1ð̋); Liberia, Sinoe County, 6.5 km NW Jacksonville, Forest near Solve Problem Vill. (103m) 23-27.I.2018 (MV Light Trap), M. Geiser, S. Safian, G. Simonics (4ð̋, 2 ) $($ ANHRT).

Distribution. Previously known from Cameroon, Democratic Republic of the Congo and Côte d'Ivoire (Massa et al. 2018), now recorded also from Liberia.

\section{Morgenia plurimaculata Massa et Moulin, 2018}

Material examined. Liberia, Lofa County, Foya Proposed Protected Area (530m) 10-19.XI.2017 (MV Light Trap), M. Aristophanous, S. Safian, G. Simonics, L. Smith (1ठ̋) (ANHRT).

Distribution. Previously known from Central African Republic and Gabon (Massa 2021a), here recorded also from Liberia.

\section{Tribe Vossiini Cadena-Castañeda, 2015}

\section{Azamia biplagiata Bolívar, 1906}

Material examined. Liberia, Lofa County, Foya Proposed Protected Area (530m) 10-19.XI.2017 (MV Light Trap), M. Aristophanous, S. Safian, G. Simonics, L. Smith (5 đđ); Liberia, Lofa County, Wologizi Mts., Base Camp Forest (611m) 20.XI-1.XII.2017 (MV Light Trap), M. Aristophanous, S. Safian, G. Simonics, L. Smith (5 今); Liberia, Sinoe County, Krahn-Bassa Reserve, Juboe River, 7.5 km SW Pellokon town, 14-21.I.2018 (MV Light Trap), M. Geiser, S. Safian, G. Simonics (1 $\left.\delta^{\Uparrow}\right)$; Liberia, Sinoe County, 6.5 km NW Jacksonville, Forest near Solve Problem Vill. (103m) 23-27.I.2018 (MV Light Trap), M. Geiser, S. Safian, G. Simonics (1ठ̋) (ANHRT).

Distribution. Previously known from Cameroon, Central African Republic, Democratic Republic of Congo, Gabon, Ghana, Guinea and Côte d'Ivoire (Massa 2021a), here recorded also from Liberia. 


\section{Gelotopoia bicolor Brunner von Wattenwyl, 1891}

Material examined. Togo, Fazao-Malfakassa NP, Point de vue campsite (Sudanian Savannah) (415m) 1623.VIII.2018 (MV Light Trap), M. Aristophanous, M. Geiser, P. Moretto, B. Sanbena (7ð̄, 2 fakassa NP, Mare aux crocodiles campsite (Sudanian Savannah/dry Forest) (505m) 26.VIII-7.IX.2018 (MV Light Trap), M. Aristophanous, M. Geiser, P. Moretto, B. Sanbena (10^, 69); Guinea, Dalaba, Forêt de Goubel (1413m) (MV Light Trap) 17.IX.2019, M. Geiser, M. Leno, S. Koivagui, W. Miles, L. Mulvaney, S. Safian (1 $\overbrace{}^{\Uparrow}$ ) (ANHRT).

Distribution. Previously known from from Sierra Leone, Côte d'Ivoire, Cameroon, Central African Republic and Zambia (Massa 2021a), now recorded also from Togo and Guinea.

\section{Tribe Ducetiini Brunner von Wattenwyl, 1878}

\section{Ducetia fuscopunctata Chopard, 1954}

Material examined. Liberia, Lofa County, Foya Proposed Protected Area (530m) 10-19.XI.2017 (MV Light Trap), M. Aristophanous, S. Safian, G. Simonics, L. Smith (1ठ); Guinea, Guinée forestière, Bossou For. \& Inst. Rech. Env. Bossou (Lowland Forest-Farmland) (690m) (gen. coll.) 24-31.VI.2019, V. Derozier, J. Suha Dore, S. Koivagui, W. Miles, S. Safian, R. Warner (1§); Guinea, Guinée forestière, Bossou For. \& Inst. Rech. Env. Bossou (Lowland Forest-Farmland) (690m) (Lepiled Light Trap) 24-31.VI.2019, V. Dérozier, J. Suha Dore, S. Koivagui, W. Miles, S. Safian, R. Warner (1今̂) (ANHRT).

Distribution. Previously known from Guinea, Sierra Leone, Ghana, Côte d'Ivoire and Togo (Massa 2016), here recorded also from Liberia.

\section{Tribe uncertain}

Bongeia puncticollis Sjöstedt, 1902

Material examined. Liberia, Lofa County, Wologizi Mts., Ridge Camp 24-29.XI.2017 (Cold Cathode Light Trap), M. Aristophanous, S. Safian, G. Simonics, L. Smith (1ठ) (ANHRT).

Distribution. Previously known from Cameroon, Central African Republic and Gabon (Massa 2021a), now recorded also from Liberia.

\section{Gabonella cothurnata (Bolívar, 1906)}

Material examined. Côte d'Ivoire, Abidjan, Banco Forest (Banco PN) (39-48m) (MV Light Trap) 21-30.IV.2017, A. Aristophanous, M. Aristophanous, M. Geiser, P. Moretto (1ð̂); Côte d'Ivoire, Abidjan, Banco Forest (Banco PN) (39-48m) (gen. coll.) 21.IV-1.V.2017, A. Aristophanous, M. Aristophanous, M. Geiser, P. Moretto (1ठ) (ANHRT).

Distribution. Described from Cameroon, known also from Gabon (as its synonym Himerta feana Griffini, 1906) and Democratic Republic of Congo (Ragge 1980), newly recorded from Côte d'Ivoire.

\section{Subfamily Mecopodinae Walker, 1871}

Tribe Mecopodini Walker, 1871

Afromecopoda austera (Karsch, 1893)

Material examined. Liberia, Sinoe County, Krahn-Bassa Reserve, Juboe River, 7.5 km SW Pellokon town, 1322.I.2018 (General Collection), M. Geiser, S. Safian, G. Simonics (1 ㅇ) (ANHRT).

Distribution. This species has been described from Togo, here is recorded also from Liberia. 



FIGS. 41-44. Lateral view of Enochletica ostentatrix Karsch, 1896 (41); Preussia lobatipes Karsch, 1890 (42); Weissenbornia praestantissima Karsch, 1888 (43); Brycoptera lobata Ragge, 1981 (44). 


\section{Euthypoda brunneotestacea Chopard, 1954}

Material examined. Côte d'Ivoire, Man, Mt. Tonkoui (1171m) 16.III.2017 (1 $\overbrace{}^{\Uparrow})$ (light) (BMPC); Côte d'Ivoire, Mt. Nimba camp, closed forest (801m) (gen. coll.) 28.IV-8.V.2016, M. Aristophanous, M. Geiser, P. Moretto (1 ${ }^{\lambda}$, 1ㅇ) (ANHRT).

Distribution. Previously known only from Mt. Nimba (Chopard 1954), here recorded also from Mt. Tonkoui, which revealed to be an important biodiversity hotpsot.

\section{Tettigoniidae as indicators of biodiversity hotspots}

Probably, while in the northern emisphere, the biodiversity crisis and the decline of insects appear to be due to climate change and to use of pesticides in agriculture, in the tropical forests of Africa, the decline of insects may depend mainly on the forest destruction. Sánchez-Bayo \& Wyckhuys (2019), consulting 73 papers that deal with the decline of insects, have observed a sharp decline that can lead to the extinction of $40 \%$ of insects in the world in the next few decades. Most of studies used by them are located in the North Europe and North America, only single papers concern tropical areas. In particular, in terrestrial ecosystems butterflies (Lepidoptera), bees and bumblebees (Hymenoptera) and coprophagous beetles (Coleoptera Scarabaeidae) were the most vulnerable species, while the major groups of aquatic insects (Odonata, Trichoptera and Ephemeroptera) have already lost a considerable amount of species. Only a small number of species are increasing; they are arthropods with a wide ecological niche and very adaptable, generalists that live in many ecosystems, adapting to different ecological niches.

Guinean forest of West Africa represent one of the world areas containing the highest number of endemic species. Table 1 lists all the species of Tettigoniidae of the subfamilies Pseudophyllinae, Conocephalinae, Hexacentrinae, Phaneropterinae, Mecopodinae and Hetrodinae that have been recorded in the concerned African areas. The total number of Tettigoniidae of the subfamilies here considered amounts to 332 in the area of Guinean forests plus Central African Republic and Gabon; 242 of them live in the Cameroon-Nigeria subregion plus Central African Republic and Gabon, 216 in the subregion of Upper Guinea. In the Upper Guinean subregion 87 (40.3\%) species resulted to be endemic, while in the Cameroon-Nigeria plus Central African Republic and Gabon 87 (35.9\%) species are endemic (Table 1); in both cases the occurrence of endemic taxa resulted higher than in plants and vertebrata (only except for amphibians), which, following Mittermeier et al. (2011) are: plants 20\%, mammals 14.9\%, birds $9.4 \%$, reptiles $25.2 \%$, amphibians $38.4 \%$, freshwater fishes $27.9 \%$. Out of 332 species, $122(36.7 \%$ ) have a wide distribution, covering West and Central Africa, and 32 (9.6\%) species living in the Cameroon-Nigeria subregion, but not in the Upper Guinean subregion, are also distributed in other areas of central or east Africa, mainly in the Democratic Republic of Congo.

However, Tettigoniidae living in Tropical Africa have the highest richness in the central-eastern and southern countries; for example some tribes are absent in the Guinean forests [the tribe Agraeciini Redtenbacher, 1891 of the subfamily Conocephalinae is present with only one species, Anthracopsis gigliotosi Karny, 1907; the tribe Eugastrini Karsch, 1887 of the subfamily Hetrodinae has only one representative, Spalacomimus liberianus (La Baume, 1911)] and some genera of Phaneropterinae, represented by many species, are only restricted to the eastern and southern Africa. The only exception seems to be that of the subfamily Pseudophyllinae, which, following Orthoptera Species File online (Cigliano et al. 2021), in tropical Africa consists of 118 species (subspecies are here excluded), 71 $(60.2 \%)$ of them only present in Central-West tropical Africa. However, from the biogeographical point of view, in tropical Africa an east-west species impoverishment occurs, with the lowest values of species richness just in the isolated West Guinean forests, but also a high percentage of endemism occurrence in West forests.

The number of Tettigoniidae species in single hotspots resulted very high, also compared with similar studies (cf. Table 1): Mt. Tonkoui (Côte d'Ivoire): 81 (present study); Taï National Park (Côte d'Ivoire): 88 (present study); Dzanga-Ndoki National Park (Central African Republic): 134 (Massa et al. 2020, present study); Miombo Woodlands (Tanzania): 56 (Hemp \& Heller 2019); Gorongosa Park (Mozambique): 55 (Naskrecki \& Guta 2019). This is the probable result of intense researches carried out with the aid of the light trap. 
TABLE 1. List of species presently known in the Central Africa (Guinean forests of the subregion Nigeria-Cameron plus biodiversity hotspots of Gabon and Central African Republic) and West Africa (Guinean forests of subregion upper Guinea) (References: Massa 2013, 2016, 2017, 2020, 2021a, 2021b, Hemp \& Massa 2017, 2021, Massa et al. 2018, 2020 ); * = endemic taxa (species living in a geographically confined territory). $\mathrm{Nd}=$ species found in the Dzanga-Ndoki National Park, Central African Republic; Ta = species found in the Taï Forest National Park, Côte d'Ivoire; To = species found in the hotspot of Mt. Tonkoui, Côte d'Ivoire.

\begin{tabular}{|c|c|c|}
\hline \multirow[t]{2}{*}{ Taxa } & \multicolumn{2}{|c|}{ Guinean Forest hotspots } \\
\hline & $\begin{array}{c}\text { Subregion Nigeria-Cameroon }+ \\
\text { Central African Republic and } \\
\text { Gabon }\end{array}$ & $\begin{array}{c}\text { Subregion Upper } \\
\text { Guinea }\end{array}$ \\
\hline Subfamily Pseudophyllinae Burmeister, 1838 & & \\
\hline Adapantus (Adapantus) bardus Karsch, 1891 & $\mathrm{X}(\mathrm{Nd})$ & $\mathrm{X}$ \\
\hline Adapantus (Adapantus) longipennis Beier, 1954 & $\mathrm{X}^{*}$ & \\
\hline Adapantus (Adapantus) osorioi (Bolívar, 1886) & $\mathrm{X}$ & $\mathrm{X}(\mathrm{Ta})$ \\
\hline Adapantus (Adapantus) transmarinus (Krauss, 1890) & & $\mathrm{X}^{*}$ \\
\hline Adapantus (Neoadapantus) affluens Naskrecki, 2008 & & $\mathrm{X}^{*}(\mathrm{To}, \mathrm{Ta})$ \\
\hline Adapantus (Neoadapantus) angulatus Naskrecki, 2008 & & $X^{*}(\mathrm{Ta})$ \\
\hline Adapantus (Neoadapantus) marmoratus Chopard, 1954 & & $\mathrm{X}^{*}(\mathrm{To})$ \\
\hline Adapantus (Neoadapantus) nitens (Chopard, 1954) & & $\mathrm{X}^{*}(\mathrm{To}, \mathrm{Ta})$ \\
\hline Adapantus (Neoadapantus) pragerorum Naskrecki, 2008 & & $\mathrm{X}^{*}$ \\
\hline Adenes albifrons Brunner von Wattenwyl, 1895 & & $\mathrm{X}^{*}$ \\
\hline Adenes gravidus Karsch, 1891 & & $\mathrm{X}^{*}$ \\
\hline Adenes obesus Karsch, 1891 & $\mathrm{X}$ & $\mathrm{X}$ \\
\hline Batodromeus herinaceus (Karsch, 1896) & $X^{*}$ & \\
\hline Batodromeus richardi (Griffini, 1908) & & $\mathrm{X}^{*}(\mathrm{To}, \mathrm{Ta})$ \\
\hline Batodromeus subulo (Karsch, 1893) & $X^{*}$ & \\
\hline Chondrodera notatipes Karsch, 1890 & $\mathrm{X}$ & \\
\hline Chondrodera ocellata Beier, 1954 & $\mathrm{X}(\mathrm{Nd})$ & $\mathrm{X}(\mathrm{To})$ \\
\hline Chondrodera subvitrea Karsch, 1891 & $\mathrm{X}(\mathrm{Nd})$ & \\
\hline Chondrodera vittifer (Walker, 1871) & $\mathrm{X}$ & $\mathrm{X}(\mathrm{Ta})$ \\
\hline Cymatomera argillata Karsch, 1891 & $\mathrm{X}$ & $\mathrm{X}(\mathrm{To}, \mathrm{Ta})$ \\
\hline Cymatomera chopardi Naskrecki, 2008 & & $\mathrm{X}^{*}$ \\
\hline Desaulcya ampulla Brunner von Wattenwyl, 1895 & $\mathrm{X}^{*}(\mathrm{Nd})$ & \\
\hline Desaulcya pictipennis Bolívar, 1906 & $X^{*}$ & \\
\hline Habrocomes lanosus Karsch, 1891 & & $X^{*}(\mathrm{Ta})$ \\
\hline Habrocomes marmoratus (Bolívar, 1906) & $X^{*}$ & \\
\hline Habrocomes p. personatus (Sjöstedt, 1901) & $\mathrm{X}^{*}(\mathrm{Nd})$ & \\
\hline Hoplidostylus argillatus Karsch, 1893 & & $\mathrm{X}^{*}$ \\
\hline Hoplidostylus borrei (Griffini, 1908) & $X^{*}$ & \\
\hline Lagarodes facetus Karsch, 1891 & $\mathrm{X}(\mathrm{Nd})$ & $\mathrm{X}(\mathrm{To}, \mathrm{Ta})$ \\
\hline Lichenochrus crassipes Karsch, 1890 & $\mathrm{X}$ & $\mathrm{X}(\mathrm{To}, \mathrm{Ta})$ \\
\hline Lichenochrus decoloratus (Brunner von Wattenwyl, 1895) & $\mathrm{X}$ & \\
\hline Lichenochrus marmoratus Sjöstedt, 1901 & $\mathrm{X}$ & $\mathrm{X}(\mathrm{Ta})$ \\
\hline Lichenochrus congicus Rehn, 1914 & $\mathrm{X}$ & $\mathrm{X}$ \\
\hline Lichenochrus servus Beier, 1954 & & $\mathrm{X}^{*}$ \\
\hline Liocentrum aduncum Karsch, 1891 & $\mathrm{X}$ & $\mathrm{X}(\mathrm{To}, \mathrm{Ta})$ \\
\hline Liocentrum hancocki Bolívar, 1906 & $\mathrm{X}$ & $\mathrm{X}(\mathrm{Nd})$ \\
\hline
\end{tabular}




\begin{tabular}{|c|c|c|}
\hline \multirow[t]{2}{*}{ Taxa } & \multicolumn{2}{|c|}{ Guinean Forest hotspots } \\
\hline & $\begin{array}{c}\text { Subregion Nigeria-Cameroon }+ \\
\text { Central African Republic and } \\
\text { Gabon }\end{array}$ & $\begin{array}{c}\text { Subregion Upper } \\
\text { Guinea }\end{array}$ \\
\hline Liocentrum rubripes Bolívar, 1906 & $\mathrm{X}$ & $\mathrm{X}$ \\
\hline Micta spinosula Karsch, 1896 & & $\mathrm{X}^{*}$ \\
\hline Mormotus alonsae Naskrecki, 2008 & & $\mathrm{X}^{*}(\mathrm{To}, \mathrm{Ta})$ \\
\hline Mormotus angustus Brunner von Wattenwyl, 1895 & $\mathrm{X}$ & $\mathrm{X}$ \\
\hline Mormotus clavaticercus Karsch, 1891 & & $\mathrm{X}^{*}(\mathrm{To}, \mathrm{Ta})$ \\
\hline Mormotus curvicauda Brunner von Wattenwyl, 1895 & $\mathrm{X}^{*}$ & \\
\hline Mormotus erectistylus Karsch, 1896 & & $X^{*}$ \\
\hline Mormotus montesi (Bolívar, 1886) & $\mathrm{X}(\mathrm{Nd})$ & $\mathrm{X}(\mathrm{Ta})$ \\
\hline Mormotus obtusatus Brunner von Wattenwyl, 1895 & & $X^{*}$ \\
\hline Mormotus ornatus Beier, 1973 & & $\mathrm{X}^{*}(\mathrm{To})$ \\
\hline Mormotus rastricercus Karsch, 1891 & & $\mathrm{X}^{*}(\mathrm{Ta})$ \\
\hline Mormotus scapularis Bolívar, 1906 & $\mathrm{X}$ & $\mathrm{X}$ \\
\hline Mustius afzelii Stål, 1873 & $\mathrm{X}(\mathrm{Nd})$ & $\mathrm{X}$ \\
\hline Mustius eurypterus Karsch, 1896 & $\mathrm{X}(\mathrm{Nd})$ & $\mathrm{X}(\mathrm{To})$ \\
\hline Mustius inversus Brunner von Wattenwyl, 1895 & $\mathrm{X}^{*}$ & \\
\hline Mustius serrulatus Bolívar, 1906 & $X$ & $\mathrm{X}$ \\
\hline Mustius superbus Sjöstedt, 1902 & $\mathrm{X}(\mathrm{Nd})$ & $\mathrm{X}(\mathrm{To})$ \\
\hline Opisthodicrus cochlearistylus Karsch, 1891 & $\mathrm{X}(\mathrm{Nd})$ & $\mathrm{X}(\mathrm{To}, \mathrm{Ta})$ \\
\hline Oxyaspis senegalensis (Bolívar, 1886) & & $\mathrm{X}^{*}$ \\
\hline Oxyaspis congensis Brunner von Wattenwyl, 1895 & $\mathrm{X}$ & $\mathrm{X}(\mathrm{To})$ \\
\hline Pantecphylus cerambycinus Karsch, 1891 & $\mathrm{X}$ & $\mathrm{X}$ \\
\hline Pantecphylus kamerunus Schmidt, 2003 & $X^{*}$ & \\
\hline Paralichenochrus turpis (Brunner von Wattenwyl, 1895) & & $X^{*}$ \\
\hline Paralichenochrus villosipes (Griffini, 1908) & $\mathrm{X}$ & \\
\hline Perteus pellucidus Bolívar, 1906 & $\mathrm{X}^{*}$ & \\
\hline Polyglochin peculiaris Karsch, 1891 & & $\mathrm{X}^{*}(\mathrm{To})$ \\
\hline Rhinodera spinifrons Beier, 1955 & $\mathrm{X}^{*}(\mathrm{Nd})$ & \\
\hline Stenampyx annulicornis Karsch, 1891 & $\mathrm{X}(\mathrm{Nd})$ & $\mathrm{X}(\mathrm{To}, \mathrm{Ta})$ \\
\hline Stizoscepa basinotata Karsch, 1896 & $\mathrm{X}$ & $\mathrm{X}$ \\
\hline Stizoscepa severini Griffini, 1908 & $\mathrm{X}^{*}$ & \\
\hline Tomias (Semiophygas) arescus (Karsch, 1896) & $\mathrm{X}$ & $\mathrm{X}$ \\
\hline Tomias (Semiophygas) gerriesmithae Naskrecki, 2008 & & $\mathrm{X}^{*}$ \\
\hline Tomias (Semiophygas) hadrus (Karsch, 1896) & $\mathrm{X}$ & $\mathrm{X}$ \\
\hline Tomias (Tomias) stenopterus Karsch, 1891 & $\mathrm{X}(\mathrm{Nd})$ & $\mathrm{X}$ \\
\hline Tympanocompus acclivis Karsch, 1891 & $\mathrm{X}(\mathrm{Nd})$ & $\mathrm{X}$ \\
\hline Tympanocompus erectistylus (Karsch, 1896) & & $\mathrm{X}^{*}$ \\
\hline Zabalius albifasciatus (Karsch, 1896) & $\mathrm{X}^{*}$ & \\
\hline Zabalius apicalis (Bolívar, 1886) & $\mathrm{X}$ & $\mathrm{X}(\mathrm{Ta})$ \\
\hline Zabalius aridus (Walker, 1869) & $\mathrm{X}$ & $\mathrm{X}(\mathrm{Ta})$ \\
\hline Zabalius girardi Beier, 1973 & & $\mathrm{X}^{*}$ \\
\hline
\end{tabular}


Zabalius lineolatus (Stål, 1873)

Zabalius robustus Beier, 1954

Subfamily Conocephalinae Burmeister, 1838

Anthracopsis gigliotosi Karny, 1907

Clasma parcispinosa Karsch, 1893

Conocephalus (Conocephalus) conocephalus (Linnaeus, 1767)

Conocephalus (Anisoptera) armatipes (Karsch, 1893)

Conocephalus (Anisoptera) brevicercus (Karsch, 1893)

Conocephalus (Anisoptera) iris (Serville, 1838)

Conocephalus (Anisoptera) maculatus (Le Guillou, 1841)

Conocephalus (Anisoptera) meadowsae Harz, 1970

Conocephalus (Chloroxiphidion) laetus (Redtenbacher, 1891)

Euconocephalus afer afer (Karny, 1907)

Lanista africana (Walker, 1871)

Lanista affinis Bolívar, 1906

Lanista crassicollis Bolívar, 1906

Plastocorypha nigrifrons (Redtenbacher, 1891)

Plastocorypha vandikana Karsch, 1896

Pseudorhynchus crosskeyi Ragge, 1969

Pseudorhynchus lanceolatus (Fabricius, 1765)

Pseudorhynchus pungens (Schaum, 1853)

Pseudorhynchus raggei nomen novum

Ruspolia baileyi Otte, 1997

Ruspolia basiguttata (Bolívar, 1906)

Ruspolia differens (Serville, 1838)

Ruspolia fuscopunctata (Karny, 1907)

Ruspolia jaegeri (Roy, 1971)

Thyridorhoptrum baileyi Pitkin, 1977

Thyridorhoptrum carbonarium (Redtenbacher, 1891)

Thyridorhoptrum senegalense (Krauss, 1877)

Subfamily Hexacentrinae Karny, 1925

Hexacentrus alluaudi Bolívar, 1906

Hexacentrus dorsatus Redtenbacher, 1891

Hexacentrus inflatus Redtenbacher, 1891

Subfamily Phaneropterinae Burmeister, 1838, with open tympana

Bueacola cornigera Sjöstedt, 1912

Catoptropteryx afra (Karsch, 1889)

Catoptropteryx ambigua Huxley, 1970

Catoptropteryx apicalis Bolívar, 1893

$\mathrm{X}(\mathrm{Nd})$
$\mathrm{X}$
$\mathrm{X}^{*}$

$\mathrm{X}(\mathrm{Nd})$

$\mathrm{X}^{*}$

$\mathrm{X}$ (To, Ta)

$\mathrm{X}^{*}$

$\mathrm{X}^{*}$

$\mathrm{X}(\mathrm{Nd})$

$\mathrm{X}(\mathrm{Nd})$

$\mathrm{X}$ (To, Ta)

$\mathrm{X}^{*}$

$\mathrm{X}$

$\mathrm{X}$

$\mathrm{X}$

$\mathrm{X}(\mathrm{Nd})$

$\mathrm{X}$ (To)

$\mathrm{X}^{*}$

$\mathrm{X}^{*}$

$\mathrm{X}^{*}$

$\mathrm{X}$

$\mathrm{X}$

$\mathrm{X}^{*}$

$\mathrm{X}$

$\mathrm{X}$ (To)

$\mathrm{X}$

$\mathrm{X}$ (To)

$\mathrm{X}^{*}$ (To)

$\mathrm{X}^{*}$

$\mathrm{X}$

$\mathrm{X}$

$\mathrm{X}(\mathrm{Nd})$

X (Ta)

$\mathrm{X}(\mathrm{Nd})$

X (Ta)

$\mathrm{X}^{*}$

$\mathrm{X}$

$\mathrm{X}$

$X^{*}(\mathrm{To}, \mathrm{Ta})$

$\mathrm{X}(\mathrm{Nd})$

X (To, Ta)

$\mathrm{X}^{*}$

$\mathrm{X}^{*}(\mathrm{Nd})$

$\mathrm{X}^{*}$

$\mathrm{X}^{*}$

$\mathrm{X}^{*}$

$\mathrm{X}(\mathrm{Nd})$

$\mathrm{X}(\mathrm{Ta})$

$\mathrm{X}(\mathrm{Nd})$
X (To, Ta) 


\begin{tabular}{|c|c|c|}
\hline \multirow[t]{2}{*}{ Taxa } & \multicolumn{2}{|c|}{ Guinean Forest hotspots } \\
\hline & $\begin{array}{c}\text { Subregion Nigeria-Cameroon }+ \\
\text { Central African Republic and } \\
\text { Gabon }\end{array}$ & $\begin{array}{c}\text { Subregion Upper } \\
\text { Guinea }\end{array}$ \\
\hline Catoptropteryx capreola Karsch, 1896 & $\mathrm{X}(\mathrm{Nd})$ & $\mathrm{X}(\mathrm{To}, \mathrm{Ta})$ \\
\hline Catoptropteryx extensipes Karsch, 1896 & $\mathrm{X}(\mathrm{Nd})$ & $\mathrm{X}(\mathrm{To}, \mathrm{Ta})$ \\
\hline Catoptropteryx guttatipes Karsch, 1890 & $\mathrm{X}(\mathrm{Nd})$ & $\mathrm{X}(\mathrm{To}, \mathrm{Ta})$ \\
\hline Catoptropteryx lineata $\mathbf{n} . \mathbf{s p .}$ & & $X^{*}$ \\
\hline Catoptropteryx nana Huxley, 1970 & $\mathrm{X}(\mathrm{Nd})$ & $\mathrm{X}(\mathrm{Ta})$ \\
\hline Catoptropteryx naevia Huxley, 1970 & $\mathrm{X}$ & $\mathrm{X}(\mathrm{To}, \mathrm{Ta})$ \\
\hline Catoptropteryx neutralipennis Karsch, 1896 & $\mathrm{X}$ & $\mathrm{X}(\mathrm{Ta})$ \\
\hline Catoptropteryx occidentalis Huxley, 1970 & $\mathrm{X}(\mathrm{Nd})$ & $\mathrm{X}$ \\
\hline Catoptropteryx punctulata (Karsch, 1890) & $\mathrm{X}(\mathrm{Nd})$ & $\mathrm{X}(\mathrm{To}, \mathrm{Ta})$ \\
\hline Catoptropteryx ramulosa Huxley, 1970 & $\mathrm{X}$ & \\
\hline Corycomima camerata (Karsch, 1889) & $\mathrm{X}^{*}(\mathrm{Nd})$ & \\
\hline Dannfeltia nana Sjöstedt, 1902 & $\mathrm{X}(\mathrm{Nd})$ & $\mathrm{X}(\mathrm{To})$ \\
\hline Diogena fausta (Burmeister, 1838) & $\mathrm{X}(\mathrm{Nd})$ & $\mathrm{X}(\mathrm{To}, \mathrm{Ta})$ \\
\hline Dithela acuticercus Sjöstedt, 1912 & $X^{*}$ & \\
\hline Dithela rectiloba Karsch, 1890 & $\mathrm{X}^{*}$ & \\
\hline Ducetia crosskeyi Ragge, 1961 & $\mathrm{X}$ & $\mathrm{X}$ \\
\hline Ducetia fuscopunctata Chopard, 1954 & & $X^{*}(\mathrm{To})$ \\
\hline Ducetia loosi Griffini, 1908 & $\mathrm{X}(\mathrm{Nd})$ & \\
\hline Eulioptera atypica Massa, 2021 & & $X^{*}$ \\
\hline Eulioptera iolandae Massa, 2021 & & $X^{*}$ \\
\hline Eulioptera crosskeyi Ragge, 1968 & $\mathrm{X}^{*}$ & \\
\hline Eulioptera disparidens Ragge, 1980 & $\mathrm{X}^{*}$ & \\
\hline Eulioptera incisa Ragge, 1980 & & $X^{*}$ \\
\hline Eulioptera spinulosa Ragge, 1956 & $\mathrm{X}$ & \\
\hline Eulioptera umbilima Ragge, 1980 & & $X^{*}$ \\
\hline Eurycoplangiodes sanghaensis Massa, 2020 & $\mathrm{X}(\mathrm{Nd})$ & $\mathrm{X}(\mathrm{To}, \mathrm{Ta})$ \\
\hline Eurycorypha adicra Karsch, 1892 & $\mathrm{X}^{*}$ & \\
\hline Eurycorypha aequatorialis Krauss, 1890 & & $X^{*}$ \\
\hline Eurycorypha canaliculata Karsch, 1890 & $\mathrm{X}(\mathrm{Nd})$ & \\
\hline Eurycorypha aff. cuspidata Krauss, 1901 & $\mathrm{X}$ & $\mathrm{X}(\mathrm{To}, \mathrm{Ta})$ \\
\hline Eurycorypha flavescens (Walker, 1869) & $\mathrm{X}$ & $\mathrm{X}(\mathrm{To})$ \\
\hline Eurycorypha klaptoczi Karny, 1917 & & $X^{*}(\mathrm{To})$ \\
\hline Eurycorypha montana Sjöstedt, 1902 & $\mathrm{X}$ & \\
\hline Eurycorypha mutica Karsch, 1891 & $\mathrm{X}$ & $\mathrm{X}$ \\
\hline Eurycorypha ndokiensis Massa, 2016 & $\mathrm{X}(\mathrm{Nd})$ & $\mathrm{X}(\mathrm{Ta})$ \\
\hline Eurycorypha ornatipes Karsch, 1890 & $\mathrm{X}$ & $\mathrm{X}(\mathrm{Ta})$ \\
\hline Eurycorypha spinulosa Karsch, 1889 & $\mathrm{X}(\mathrm{Nd})$ & \\
\hline Eurycorypha sp. 1 & $\mathrm{X}^{*}(\mathrm{Nd})$ & \\
\hline Eurycorypha sp. 2 & $\mathrm{X}^{*}(\mathrm{Nd})$ & \\
\hline Eurycorypha sp. 3 & $\mathrm{X}^{*}(\mathrm{Nd})$ & \\
\hline Eurycorypha sp. 4 & & $\mathrm{X}^{*}(\mathrm{To})$ \\
\hline
\end{tabular}


Eurycorypha sp. 5

Eurycorypha sp. 6

Eurycorypha sp. 7

Eurycorypha strangulata (Walker, 1869)

Eurycorypha sudaniensis Giglio-Tos, 1907

Eurycorypha stylata Stål, 1873

Eurycorypha velicauda Karsch, 1893

Eurycorypha zebrata Bruner, 1920

Gabonella cothurnata (Bolívar, 1906)

Gelotopoia bicolor Brunner von Wattenwyl, 1891

Griffinipteryx mukonja (Griffini, 1908)

Monteiroa nigricauda Ragge, 1980

Oxygonatium huxleyi Ragge, 1980

Paraeulioptera emitflesti Massa, 2020

Paraeurycorypha ocellata Massa et Annoyer, 2020

Phaneroptera abdita Massa, 2021

Phaneroptera maculosa Ragge, 1956

Phaneroptera magna Ragge, 1956

Phaneroptera sparsa Stål, 1857

Pigalua insularis (Ragge, 1980)

Plangia astylata $\mathbf{n}$. sp.

Plangia chopardi $\mathbf{n}$. sp.

Plangia deminuta Griffini, 1908

Plangia karschi Chopard, 1954

Plangia nebulosa Karsch, 1890

Plangia villiersi Chopard, 1954

Pleothrix conradti (Bolívar, 1906)

Pseudoplangia laminifera (Karsch, 1896)

Scolocerca fusciala Ragge, 1980

Scolocerca thomasi Massa, 2021

Tropidophrys amydra Karsch, 1896

Subfamily Phaneropterinae Burmeister, 1838, with

$$
\text { closed tympana }
$$

Arantia (Arantia) angustipennis Chopard, 1954

Arantia (Arantia) brevipes Chopard, 1954

Arantia (Arantia) fatidica (Stål, 1873)

Arantia (Arantia) gretae Massa, 2020

Arantia (Arantia) hydatinoptera Karsch, 1889

Arantia (Arantia) ivoriana Hemp et Massa, 2017

Arantia (Arantia) leptocnemis Karsch, 1890

Arantia (Arantia) manca Bolívar, 1906

\begin{tabular}{|c|c|}
\hline & $\mathrm{X}^{*}(\mathrm{Ta})$ \\
\hline & $\mathrm{X}^{*}(\mathrm{Ta})$ \\
\hline & $\mathrm{X}^{*}$ \\
\hline & $\mathrm{X}^{*}$ \\
\hline \multicolumn{2}{|l|}{$X$} \\
\hline$X$ & $\mathrm{X}(\mathrm{To}, \mathrm{Ta})$ \\
\hline$X$ & $\mathrm{X}(\mathrm{To})$ \\
\hline \multicolumn{2}{|l|}{$X^{*}$} \\
\hline $\mathrm{X}$ & $X$ \\
\hline $\mathrm{X}(\mathrm{Nd})$ & $\mathrm{X}(\mathrm{To}, \mathrm{Ta})$ \\
\hline \multicolumn{2}{|l|}{$\mathrm{X}^{*}$} \\
\hline $\mathrm{X}(\mathrm{Nd})$ & X (Ta) \\
\hline $\mathrm{X}(\mathrm{Nd})$ & $X$ \\
\hline \multicolumn{2}{|l|}{$\mathrm{X}^{*}(\mathrm{Nd})$} \\
\hline \multicolumn{2}{|l|}{$\mathrm{X}^{*}(\mathrm{Nd})$} \\
\hline & $\mathrm{X}^{*}$ \\
\hline $\mathrm{X}(\mathrm{Nd})$ & $\mathrm{X}$ \\
\hline \multicolumn{2}{|l|}{$X$} \\
\hline \multirow[t]{2}{*}{$\mathrm{X}(\mathrm{Nd})$} & $\mathrm{X}(\mathrm{To}, \mathrm{Ta})$ \\
\hline & $\mathrm{X}^{*}$ \\
\hline \multicolumn{2}{|l|}{$\mathrm{X}^{*}(\mathrm{Nd})$} \\
\hline & $\mathrm{X}^{*}$ \\
\hline \multirow[t]{2}{*}{$\mathrm{X}(\mathrm{Nd})$} & X (To, Ta) \\
\hline & $\mathrm{X}^{*}(\mathrm{To}, \mathrm{Ta})$ \\
\hline $\mathrm{X}(\mathrm{Nd})$ & $\mathrm{X}$ \\
\hline $\mathrm{X}$ & $\mathrm{X}$ \\
\hline \multicolumn{2}{|l|}{$\mathrm{X}^{*}$} \\
\hline \multirow[t]{3}{*}{$\mathrm{X}(\mathrm{Nd})$} & X (Ta) \\
\hline & $\mathrm{X}^{*}$ \\
\hline & $X^{*}(\mathrm{Ta})$ \\
\hline \multirow[t]{3}{*}{$X^{*}$} & $X$ \\
\hline & $\mathrm{X}^{*}(\mathrm{To})$ \\
\hline & $\mathrm{X}^{*}(\mathrm{To}, \mathrm{Ta})$ \\
\hline$X$ & $X$ \\
\hline \multicolumn{2}{|l|}{$\mathrm{X}^{*}(\mathrm{Nd})$} \\
\hline \multirow[t]{2}{*}{$X$} & $X$ \\
\hline & $\mathrm{X}^{*}(\mathrm{Ta})$ \\
\hline \multicolumn{2}{|l|}{$X^{*}$} \\
\hline $\mathrm{X}(\mathrm{Nd})$ & $\mathrm{X}$ \\
\hline
\end{tabular}

...Continued on the next page 


\begin{tabular}{|c|c|c|}
\hline \multirow[t]{2}{*}{ Taxa } & \multicolumn{2}{|c|}{ Guinean Forest hotspots } \\
\hline & $\begin{array}{c}\text { Subregion Nigeria-Cameroon }+ \\
\text { Central African Republic and } \\
\text { Gabon }\end{array}$ & $\begin{array}{l}\text { Subregion Upper } \\
\text { Guinea }\end{array}$ \\
\hline Arantia (Arantia) marginata Massa, 2021 & $\mathrm{X}(\mathrm{Nd})$ & $\mathrm{X}(\mathrm{To}, \mathrm{Ta})$ \\
\hline Arantia (Arantia) quinquemaculata Hemp \& Massa, 2017 & $\mathrm{X}(\mathrm{Nd})$ & $\mathrm{X}(\mathrm{Ta})$ \\
\hline Arantia (Arantia) simplicinervis Karsch, 1889 & $\mathrm{X}(\mathrm{Nd})$ & $\mathrm{X}(\mathrm{To}, \mathrm{Ta})$ \\
\hline Arantia (Euarantia) bispinosa Hemp et Massa, 2017 & & $\mathrm{X}^{*}(\mathrm{Ta})$ \\
\hline Arantia (Euarantia) congensis Griffini, 1908 & $\mathrm{X}(\mathrm{Nd})$ & \\
\hline Arantia (Euarantia) excelsior Karsch, 1889 & $\mathrm{X}(\mathrm{Nd})$ & $\mathrm{X}(\mathrm{To})$ \\
\hline Arantia (Euarantia) fasciata (Walker, 1869) & $\mathrm{X}$ & $\mathrm{X}$ \\
\hline Arantia (Euarantia) griffinii Hemp \& Massa, 2017 & $\mathrm{X}^{*}(\mathrm{Nd})$ & \\
\hline Arantia (Euarantia) incerata Karsch, 1893 & $\mathrm{X}$ & $\mathrm{X}$ \\
\hline Arantia (Euarantia) latifolia Karsch, 1890 & $\mathrm{X}$ & $\mathrm{X}(\mathrm{Ta})$ \\
\hline Arantia (Euarantia) marmorata Karsch, 1889 & $\mathrm{X}(\mathrm{Nd})$ & \\
\hline Arantia (Euarantia) melanota Sjöstedt, 1902 & $\mathrm{X}(\mathrm{Nd})$ & $\mathrm{X}(\mathrm{To})$ \\
\hline $\begin{array}{l}\text { Arantia (Euarantia) rectifolia Brunner von Wattenwyl, } \\
1878\end{array}$ & $\mathrm{X}(\mathrm{Nd})$ & $\mathrm{X}(\mathrm{To}, \mathrm{Ta})$ \\
\hline Arantia (Euarantia) regina Karsch, 1889 & $\mathrm{X}(\mathrm{Nd})$ & $\mathrm{X}(\mathrm{To})$ \\
\hline Arantia (Euarantia) retinervis Karsch, 1889 & $\mathrm{X}(\mathrm{Nd})$ & $\mathrm{X}(\mathrm{To}, \mathrm{Ta})$ \\
\hline Arantia (Euarantia) scurra Karsch, 1896 & $\mathrm{X}(\mathrm{Nd})$ & $\mathrm{X}(\mathrm{Ta})$ \\
\hline Arantia (Euarantia) syssamagalei Massa et Annoyer, 2020 & $\mathrm{X}^{*}(\mathrm{Nd})$ & \\
\hline Arantia (Euarantia) tibiaspinosa Hemp et Massa, 2017 & & $\mathrm{X}^{*}(\mathrm{Ta})$ \\
\hline Arantia (Goetia) dimidiata (Bolívar, 1906) & $\mathrm{X}(\mathrm{Nd})$ & $\mathrm{X}$ \\
\hline Arantia (Goetia) galbana (Karsch, 1891) & $\mathrm{X}(\mathrm{Nd})$ & $\mathrm{X}(\mathrm{Ta})$ \\
\hline Arantia (Goetia) purpurea (Massa, 2013) & $\mathrm{X}^{*}(\mathrm{Nd})$ & \\
\hline Azamia biplagiata Bolívar, 1906 & $\mathrm{X}(\mathrm{Nd})$ & $\mathrm{X}(\mathrm{To}, \mathrm{Ta})$ \\
\hline Bongeia brevicauda Ebner, 1943 & & $\mathrm{X}^{*}$ \\
\hline Bongeia puncticollis Sjöstedt, 1902 & $\mathrm{X}(\mathrm{Nd})$ & $\mathrm{X}(\mathrm{To}, \mathrm{Ta})$ \\
\hline Brycoptera lobata Ragge, 1981 & $\mathrm{X}(\mathrm{Nd})$ & $\mathrm{X}(\mathrm{To})$ \\
\hline Buettneria maculiceps Karsch, 1889 & $\mathrm{X}$ & \\
\hline Cestromoecha tenuipes (Karsch, 1890) & $\mathrm{X}(\mathrm{Nd})$ & $X$ \\
\hline Cestromoecha longicerca Massa, 2013 & $\mathrm{X}^{*}(\mathrm{Nd})$ & \\
\hline Dapanera brevistylata Massa, 2020 & $\mathrm{X}(\mathrm{Nd})$ & $\mathrm{X}$ \\
\hline Dapanera falxcercata Massa, 2017 & $X^{*}$ & \\
\hline Dapanera genuteres Karsch, 1889 & $\mathrm{X}(\mathrm{Nd})$ & $\mathrm{X}(\mathrm{To}, \mathrm{Ta})$ \\
\hline Dapanera irregularis Karsch, 1890 & $\mathrm{X}(\mathrm{Nd})$ & $\mathrm{X}(\mathrm{To}, \mathrm{Ta})$ \\
\hline Dapanera occulta Massa, 2015 & $\mathrm{X}^{*}(\mathrm{Nd})$ & \\
\hline Drepanophyllum corrosifolium Karsch, 1896 & $X^{*}$ & \\
\hline Drepanophyllum marmoratum Karsch, 1890 & $\mathrm{X}(\mathrm{Nd})$ & \\
\hline Enochletica ostentatrix Karsch, 1896 & $\mathrm{X}(\mathrm{Nd})$ & $\mathrm{X}(\mathrm{To}, \mathrm{Ta})$ \\
\hline Gravenreuthia saturata Karsch, 1892 & $X^{*}$ & \\
\hline Horatosphaga crosskeyi Ragge, 1960 & $X$ & $\mathrm{X}$ \\
\hline Horatosphaga inclusa Karsch, 1893 & & $\mathrm{X}^{*}$ \\
\hline Itokiia sylvarum Sjöstedt, 1902 & $\mathrm{X}^{*}(\mathrm{Nd})$ & \\
\hline
\end{tabular}


Japygophana peloti Carl, 1921

Leiodontocercus angustipennis Chopard, 1954

Leiodontocercus condylus Ragge, 1962

Leiodontocercus malleus Ragge, 1962

Leiodontocercus muticus Massa, 2020

Leiodontocercus philipporum Massa, 2020

Leiodontocercus spinicercatus Massa, 2020

Leiodontocercus vicii Massa, 2020

Mangomaloba angustipennis Chopard, 1958

Mangomaloba latipennis Chopard, 1954

Mangomaloba monticola Sjöstedt, 1902

Mangomaloba royi Chopard, 1954

Morgenia angustipinnata Massa, 2018

Morgenia hamuligera Karsch, 1890

Morgenia melica Karsch, 1893

Morgenia modulata Karsch, 1896

Morgenia plurimaculata Massa et Moulin, 2018

Morgenia rubricornis Sjöstedt, 1913

Morgenia spathulifera Griffini, 1908

Myllocentrum stigmosum (Karsch, 1896)

Myllocentrum raggei Massa, 2013

Paraporeuomena signata Massa, 2018

Phlaurocentrum elegans Massa, 2013

Phlaurocentrum latevittatum Karsch, 1889

Phlaurocentrum maculatum Ragge, 1962

Phlaurocentrum mecopodoides Karsch, 1891

Phlaurocentrum morettoi Massa, 2013

Phlaurocentrum paratuberosum Massa, 2013

Phlaurocentrum tuberosum Ragge, 1962

Physocorypha politurata Karsch, 1896

Plangiola herbacea Bolívar, 1906

Plangiopsis adeps Karsch, 1896

Plangiopsis foraminata Karsch, 1891

Plangiopsis semiconchata Karsch, 1889

Poreuomena africana Brunner von Wattenwyl, 1878

Poreuomena crassipes Karsch, 1890

Poreuomena duponti Griffini, 1908

Poreuomena forcipata Sjöstedt, 1902

Poreuomena huxleyi Massa, 2013

Poreuomena ivoriana Hemp et Massa, 2021

Poreuomena laeglae (Massa, 2015)
Gabon

$\mathrm{X}^{*}$

Guinea

$\mathrm{X}^{*}(\mathrm{Ta})$

$\mathrm{X}(\mathrm{Nd})$

$\mathrm{X}^{*}$

$\mathrm{X}^{*}$

$\mathrm{X}^{*}$

$\mathrm{X}^{*}(\mathrm{Nd})$

$\mathrm{X}^{*}(\mathrm{Nd})$

$\mathrm{X}^{*}$

$\mathrm{X}^{*}$ (Ta)

$\mathrm{X}^{*}$

$\mathrm{X}^{*}$

$\mathrm{X}^{*}(\mathrm{Nd})$

$\mathrm{X}(\mathrm{Nd})$

X (To, Ta)

$\mathrm{X}(\mathrm{Nd})$

X (To, Ta)

$\mathrm{X}(\mathrm{Nd})$

X (Ta)

$\mathrm{X}(\mathrm{Nd})$

$\mathrm{X}$

$\mathrm{X}(\mathrm{Nd})$

$\mathrm{X}$ (To, Ta)

$\mathrm{X}(\mathrm{Nd})$

X (To, Ta)

$\mathrm{X}(\mathrm{Nd})$

$\mathrm{X}(\mathrm{Ta})$

$\mathrm{X}(\mathrm{Nd})$

$X(\mathrm{Ta})$

$\mathrm{X}^{*}(\mathrm{Nd})$

$\mathrm{X}^{*}(\mathrm{Nd})$

$\mathrm{X}(\mathrm{Nd})$

X (Ta)

$\mathrm{X}(\mathrm{Nd})$

$\mathrm{X}(\mathrm{Nd})$

$\mathrm{X}^{*}(\mathrm{Nd})$

$\mathrm{X}^{*}(\mathrm{Nd})$

$\mathrm{X}(\mathrm{Nd})$

$\mathrm{X}^{*}$

$\mathrm{X}^{*}(\mathrm{Nd})$

$\mathrm{X}(\mathrm{Nd})$

X (To, Ta)

$\mathrm{X}(\mathrm{Nd})$

X (To, Ta)

$\mathrm{X}(\mathrm{Nd})$

X (To, Ta)

$\mathrm{X}^{*}$

$\mathrm{X}(\mathrm{Nd})$

X

$\mathrm{X}^{*}$

$\mathrm{X}^{*}(\mathrm{Nd})$

$\mathrm{X}$

$\mathrm{X}$

$X^{*}$ (Ta)

$\mathrm{X}^{*}$ (To) 
Gabon

Poreuomena lamottei Chopard, 1954

Poreuomena magnicerca (Massa, 2013)

Poreuomena matthaei Hemp et Massa, 2021

Poreuomena sanghensis Massa, 2013

Poreuomena wilverthi Griffini, 1908

Preussia lobatipes Karsch, 1890

Symmetrokarschia africana (Brunner von Wattenwyl, 1878)

Stenamblyphyllum dilutum Karsch, 1896

Tapiena minor Bolívar, 1906

Tetraconcha annoyeri Massa, 2017

Tetraconcha aristophanousi Massa, 2017

Tetraconcha danflousi Massa, 2017

Tetraconcha fenestrata Karsch, 1890

Tetraconcha longipes (Bolívar, 1893)

Tetraconcha loubesi Massa, 2017

Tetraconcha fijalkowskii Massa, 2017

Tetraconcha fusca Massa, 2021

Tetraconcha morettoi Massa, 2017

Tetraconcha ndokiensis Massa, 2017

Tetraconcha omonomai Massa, 2017

Tetraconcha perezi Massa, 2017

Tetraconcha ruzzieri Massa, 2017

Tetraconcha smaragdina Brunner von Wattenwyl, 1878

Tetraconcha stichyrata Karsch, 1890

Tylopsis farrowi Ragge, 1972

Tylopsis irregularis Karsch, 1893

Tylopsis rubrescens Kirby, 1900

Vossia obesa Brunner von Wattenwyl, 1891

Weissenbornia praestantissima Karsch, 1888

Zeuneria biramosa Sjöstedt, 1929

Zeuneria longicercus Sjöstedt, 1929

Zeuneria melanopeza Karsch, 1889

\section{Subfamily Mecopodinae Walker, 1871}

Acridoxena hewaniana Smith, 1865

Afromecopoda austera (Karsch, 1893)

Afromecopoda frontalis (Walker, 1871)

Afromecopoda monroviana (Karsch, 1886)

Afromecopoda preussiana (Karsch, 1891)

Anoedopoda erosa Karsch, 1891

Apteroscirtus denudatus Karsch, 1891

Corycoides abruptus (Krauss, 1890)

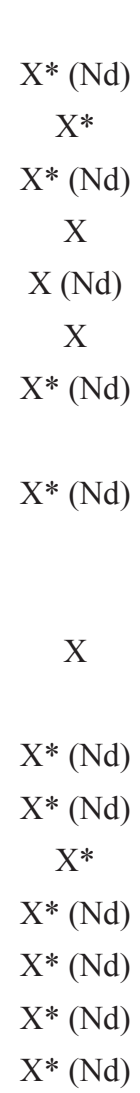

X 


\begin{tabular}{|c|c|c|}
\hline \multirow[t]{2}{*}{ Taxa } & \multicolumn{2}{|c|}{ Guinean Forest hotspots } \\
\hline & $\begin{array}{c}\text { Subregion Nigeria-Cameroon }+ \\
\text { Central African Republic and } \\
\text { Gabon }\end{array}$ & $\begin{array}{c}\text { Subregion Upper } \\
\text { Guinea }\end{array}$ \\
\hline Corycoides intermedius (Redtenbacher, 1892) & & $\mathrm{X}^{*}$ \\
\hline Corycoides jurinei (Saussure, 1892) & $\mathrm{X}^{*}$ & \\
\hline Corycoides karschi (Krauss, 1890) & $\mathrm{X}^{*}(\mathrm{Nd})$ & \\
\hline Corycoides kraussi (Kirby, 1906) & $X^{*}$ & \\
\hline Corycoides paradoxus (Bolívar, 1893) & & $X^{*}$ \\
\hline Corycoides siccifolius (Sjöstedt, 1902) & $\mathrm{X}^{*}$ & \\
\hline Euthypoda acutipennis (Karsch, 1886) & $\mathrm{X}^{*}(\mathrm{Nd})$ & \\
\hline Euthypoda bicolor (Bolívar, 1893) & & $X^{*}$ \\
\hline Euthypoda brevipennis (Redtenbacher, 1892) & & $X^{*}$ \\
\hline Euthypoda brunneotestacea Chopard, 1954 & & $\mathrm{X}^{*}(\mathrm{To})$ \\
\hline Euthypoda kanguroo (Pictet, 1888) & $\mathrm{X}$ & $\mathrm{X}$ \\
\hline Leproscirtus brunneri Karny, 1919 & $X^{*}$ & \\
\hline Leproscirtus granulosus apterus Karny, 1919 & $X^{*}$ & \\
\hline \multicolumn{3}{|c|}{ Subfamily Hetrodinae Brunner von Wattenwyl, 1878} \\
\hline Cosmoderus erinaceus (Fairmaire, 1858) & $\mathrm{X}(\mathrm{Nd})$ & \\
\hline Cosmoderus femoralis (Sjöstedt, 1902) & $X^{*}$ & \\
\hline Cosmoderus maculatus (Kirby, 1896) & $\mathrm{X}^{*}$ & \\
\hline Gymnoproctus abortivus (Serville, 1838) & & $X^{*}$ \\
\hline Spalacomimus liberianus (La Baume, 1911) & & $\mathrm{X}^{*}$ \\
\hline Total No. of taxa & 242 & 216 \\
\hline Total No. of endemic taxa (\%) & $87(35.9 \%)$ & $87(40.3 \%)$ \\
\hline
\end{tabular}

Interestingly, putting the known species in four chronological categories of description date (before 1900, between 1901 and 1950, between 1951 and 2000, 2001-today) we may have an objective information on the fact that a high percentage of species (19.9\%) has been discovered only in the present millennium (Fig. 45). Thus, very likely the number of species will grow in the next years if further entomological surveys are carried out. Naskrecki (2008) pointed out that some tropical African Orthoptera are known only from the type material and some of them probably became extinct; further, the speed with which forest habitats change suggests that many species will become extinct before they are even discovered. Extinction is the gravest consequence of the biodiversity crisis, since it is irreversible, and generally it is preceded by the rarity. We may presume that the extinction rate among the still unknown species is higher than that of the known species, since they are mostly rare species, and generally rarity is synonymous with vulnerability. Very likely species recently described on single specimens are already threatened or in verge of extinction.

Following Reid \& Miller (1989), biodiversity conservation is the management of human interactions with the variety of living forms and ecosystems, maximizing the benefits and maintaining their potential, in order to meet the needs and aspirations of future generations. However, this is possible when human populations live normally without risking their lives every day for war and disease. According to Turner et al. (2010) desperate human populations are driven toward further environmental degradation, and the forest decline is likely to be accelerated during the ongoing SARS-CoV pandemic in 2020-2021; further the Guinean area of Africa is known as one of the areas with the highest infant mortality due to malaria. The Gross Domestic Product is the single indicator considered by developed societies, the index of the "well-being" of a country. The path of continuous population growth has led us to the current frightening environmental and social situation which has caused an incredible devastation of terrestrial and marine ecosystems, the alteration of fundamental biogeochemical cycles, the dramatic loss of biodiversity, climate change and a profound social inequality, to name just some of the most evident and widely documented phenomena. 


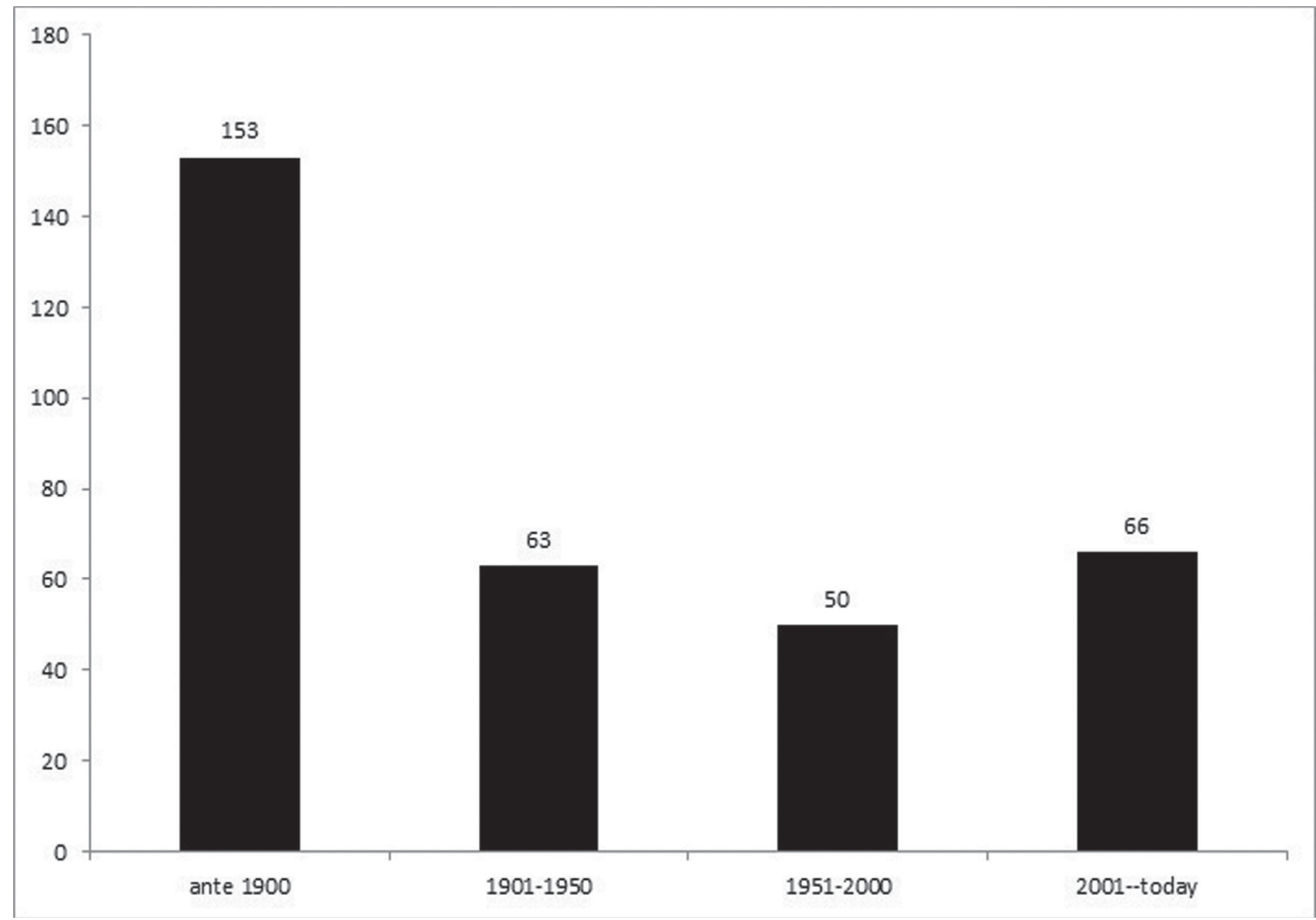

FIG. 45. Number of species of Tettigoniidae of the subfamilies Pseudophyllinae, Conocephalinae, Hexacentrinae, Phaneropterinae, Mecopodinae and Hetrodinae of central-west tropical Africa, described before 1900, between 1901 and 1950, between 1951 and 2000, and between 2001 and today.

Human activities have elevated the rate of species extinctions to a thousand or more times the natural rate (Pimm et al. 1995). The forest degradation makes fauna extremely vulnerable. In tropical Africa deforestation is the major threat to animal and plant species; plantation agriculture has been very significant in replacing forest in Côte d'Ivoire, parts of Ghana, southern Nigeria, western Cameroon and Bioko (Mittelmeier et al. 2011) and of course it is very difficult to establish how many species this deforestation has negatively involved. The breadth of the concept of biodiversity reflects the interrelationships between genes, species and ecosystems. Since genes are components of species and species of ecosystems, the alteration of the structure at each level of this hierarchy can modify the others.

\section{Acknowledgements}

This paper is dedicated to the late Philippe Annoyer, President of the Association Insectes du Monde (www.insectesdumonde.org), dear friend, entomologist, and co-organizer of many entomological expeditions to tropical African biodiversity hotspots, who regrettably passed away too early.

I am indebted with Philippe Moretto (Association Catharsius), co-organizer of the expedition Sangha 2012 (Central African Republic) and organizer of several entomological expeditions to Côte d'Ivoire; he kindly let me study the material collected during these missions and sent me very useful information and comments. I also thank very much the late Philippe Annoyer, and Samuel Danflous, Matias Loubes for their collaboration and help during the collecting nights at light in the Taï National Park (Côte d'Ivoire) in March 2017, both on the ground and on the canopy; and Vincenzo Vomero (Rome, Italy), who presented me a lot of specimens collected in Ghana in 19821985 by Pietro Butti. A warm acknowledgement goes to Claudia Hemp and Klaus-Gerhard Heller, for their useful taxonomic suggestions. 
I thank very much Richard Smith, Chairman of the African Natural History Research Trust (ANHRT) (Hereford, UK), who loaned a lot of specimens used for the present study, Hitoshi Takano, research and curator of ANHRT, who sent me important ecological information on the collecting sites, and the collectors and collaborators in Senegal, Sierra Leone, Liberia, Guinea, Togo, and Côte d'Ivoire entomological expeditions carried out by ANHRT and the Association Catharsius. Collectors were A. Aristophanous, M. Aristophanous, V. Dérozier, J.S. Dore, M. Geiser, R. Goff, S. Koivagui, M. Leno, W. Miles, S. Ouattara, Y. Outtara, P. Moretto, L. Mulvaney, S. Safian, B. Sanbena, G. Simonics, L. Smith, H. Takano, R. Warner. I would thank in particular Marios Aristophanous, William Miles, Philippe Moretto and Lydia Mulvaney, who very kindly provided some nice photographs of collecting habitats and species.

This research received support from the Synthesys Project, which is financed by European Community Research Infrastructure Action under the FP7 "Capacities" Programme at the Museo Nacional de Ciencias Naturales, Madrid (CSIC) (2013: ES-TAF-2438), the Museum für Naturkunde, Berlin (2014: DE-TAF-4109), the Naturhistorisches Museum, Vienna (2016: AT-TAF-5324), the National Museum, Prague (2016: CZ-TAF-5559) and the Royal Belgian Institute of Natural Sciences, Bruxelles (2017: BE-TAF-6319). I am especially indebted to Mercedes Paris (Museo Nacional de Ciencias Naturales of Madrid), Michael Ohl (Museum für Naturkunde of Berlin), Suzanne Randolf and Harald Bruckner (Naturhistorisches Museum, Vienna), Jérôme Constant (Royal Belgian Institute of Natural Sciences, Bruxelles), Martin Fikáček (National Museum Natural History, Prague), Laure Desutter (Muséum National d'Histoire Naturelle, Paris), Roberto Poggi (Museo di Storia Naturale 'G. Doria', Genoa), who facilitated the study of specimens preserved in their museums.

\section{References}

Atsri, H.K., Abotsi, K.E. \& Kokou, K. (2018) Enjeux écologiques de la conservation des mosaïques forêt-savane semi-montagnardes au centre du Togo (Afrique de l'Ouest). Journal of Animal and Plant Sciences, 38 (1), 6112-6128. [http://www. m.elewa.org/JAPS]

Bakarr, M., Oates, J.F., Fahr, J., Parren, M., Rödel, M.-O. \& Demey, R. (2004) Guinean forests of West Africa. In: Mittermeier, R.A., Gil, P.R., Hoffmann, M., Pilgrim, J., Brooks, T., Mittermeier, C.G., Lamoreux, J. \& da Fonesca, G.A.B. (Eds.), Hotspots Revisited: Earth's Biologically Richest and Most Endangered Terrestrial Ecoregions. Conservation International \& CEMEX, Washington, D.C., pp. 123-130

Chopard, L. (1954) La réserve naturelle integrale du Mont Nimba. II. Orthopteres Ensiferes. Memoires Institut francais Afrique noire, 40, 25-97.

Cigliano, M.M., Braun, H., Eades, D.C. \& Otte, D. (2021) Orthoptera Species File. Version 5.0/5.0. Available from: http://Orthoptera.SpeciesFile.org (accessed 11 March 2021)

Griffini, A. (1908) Phasgonuridae africane del R. Museo di Storia Naturale in Bruxelles. 6. Phaneropteridae pars 2a (reliquae species omnes). Mémoires de la Societé entomologique Belgique, 15, 201-226. [http://www.biodiversitylibrary.org/item/ 41740\#page/215/mode/1up]

Guillaumet, J.-L. \& Adjanohoun, F. (1970) La végétation de la Côte d'Ivoire. in: Le milieu naturel de la Côte d'T̂voire. 2 Annexes. Orstom, Paris, pp. 161-261.

Hadley, A. (2008) Combine Z. Available from: https://www.hadleyweb.pwp.blueyonder.co.uk (accessed 20 February 2009)

Heller, K.-G. (2006) Song Evolution and Speciation in Bushcrickets. In: Drosopoulos, S. \& Claridge, M.F. (Eds.), Insect Sounds and Communication. Taylor \& Francis, Boca Raton, London and New York, pp. 137-151. https://doi.org/10.1201/9781420039337.ch9

Heller, K.-G., Hemp, C., Liu, C. \& Volleth, M. (2014) Taxonomic, bioacoustic and faunistic data on a collection of Tettigonioidea from eastern Congo (Insecta: Orthoptera). Zootaxa, 3785 (3), 343-376. https://doi.org/10.11646/zootaxa.3785.3.2

Hemp, C. (2017) Neotype designation for Plangia graminea (Serville, 1838) and two new Plangia species from Tanzania, East Africa (Orthoptera: Tettigoniidae: Phaneropterinae). Zootaxa, 4324 (1), 180-188. https://doi.org/10.11646/zootaxa.4324.1.10

Hemp, C. \& Heller, K.-G. (2019) Orthoptera (Tettigoniidae and Acridoidea) from Miombo woodlands of Central Tanzania with the description of new taxa. Zootaxa, 4671 (2), 151-194.

https://doi.org/10.11646/zootaxa.4671.2.1

Hemp, C., Heller, K.-G., Warchalowska-Sliwa, E., Grzywacz, B. \& Hemp, A. (2015) Review of the Plangia graminea (Serville) complex and the description of new Plangia species from East Africa (Orthoptera: Phaneropteridae, Phaneropterinae) with data on habitat, bioacoustics, and chromosomes. Organisms, Diversity \& Evolution, 15 (3), 471-488. https://doi.org/10.1007/s13127-015-0216-8

Hemp, C. \& Massa, B. (2017) Review of the African genera Arantia Stål and Goetia Karsch (Orthoptera: Tettigoniidae: Phaneropterinae). Zootaxa, 4362 (4), 451-498. 
https://doi.org/10.11646/zootaxa.4362.4.1

Hemp, C. \& Massa, B. (2021) Biogeographical and evolutionary aspects of a Guineo-Congolian bushcricket tribe: Revision of the genera Cestromoecha Karsch, 1893 and Poreuomena Brunner von Wattenwyl, 1878, with the description of new species (Orthoptera, Tettigoniidae, Phaneropterinae). Deutsche Entomologische Zeitschrift, 68 (1), 45-79. https://doi.org/10.3897/dez.68.60193

Huxley, J. (1970) A revision of the genus Catoptropteryx Karsch (Orthoptera: Tettigoniidae). Bulletin British Museum natural History, Entomology, 24, 129-170. https://doi.org/10.5962/bhl.part.1520

Karsch, F.A.F. (1890) Verzeichnis der von Herrn Dr. Paul Preuss auf der Barombi-Station in Deutsch-Westafrika 1890 gesammelten Locustodeen aus den Familien der Phaneropteriden, Mekonemiden und Gryllakriden. Entomologische Nachrichten, 16 (23), 353-369. [http://www.biodiversitylibrary.org/item/81935\#page/367/mode/1up]

Massa, B. (2013) Diversity of leaf katydids (Orthoptera: Tettigoniidae: Phaneropterinae) of Dzanga-Ndoki National Park, Central African Republic, with selected records from other African countries. Journal of Orthoptera Research, 22 (2), $125-152$. https://doi.org/10.1665/034.022.0201

Massa, B. (2016) On some interesting African katydids (Orthoptera Tettigoniidae). Entomologia, 4 (303), 1-15. https://doi.org/10.4081/entomologia.2016.303

Massa, B. (2017) Revision of the tropical African genus Tetraconcha (Orthoptera: Tettigoniidae: Phaneropterinae) with the description of ten new species. Journal of Orthoptera Research, 26, 211-232. https://doi.org/10.3897/jor.26.21469

Massa, B. (2020) Revision of the Afrotropical genus Leiodontocercus (Orthoptera, Tettigoniidae, Phaneropterinae) with a description of four new species. Zookeys, 951, 47-65.

https://doi.org/10.3897/zookeys.951.53814

Massa, B. (2021a) Tettigoniidae (Insecta) collected in tropical forests of Zambia, Cameroon, Gabon and Sao Tomé during the entomological expeditions of African Natural History Research Trust. Annales de la Société entomologique de France, 57 (1), 29-76. https://doi.org/10.1080/00379271.2020.1867004

Massa, B. (2021b) Some new species of Phaneroptera, Eulioptera and Scolocerca (Orthoptera: Tettigoniidae: Phaneropterinae) from West Tropical Africa. Zootaxa, 4948 (1), 123-135. https://doi.org/10.11646/zootaxa.4948.1.7

Massa, B., Annoyer, P., Perez, C., Danflous, S. \& Duvot, G. (2020) Orthoptera Tettigoniidae (Conocephalinae, Hexacentrinae, Phaneropterinae, Mecopodinae, Hetrodinae) from some protected areas of Central African Republic. Zootaxa, 4780 (3), 401-447. https://doi.org/10.11646/zootaxa.4780.3.1

Massa, B. \& Fontana, P. (2020) Endemism in Italian Orthoptera. Biodiversity Journal, 11 (2), 405-434. https://doi.org/10.31396/Biodiv.Jour.2020.11.2.405.434

Massa, B., Heller, K.-G., Warchalowska-Sliwa, E. \& Moulin, N. (2018) The tropical African genus Morgenia (Orthoptera, Tettigoniidae, Phaneropterinae) with emphasis on the spur at the mid tibia. Deutsche Entomologische Zeitschrift, 65, 161-175. https://doi.org/10.3897/dez.65.26693

Mittermeier, R.A., Gil, P.R., Hoffmann, M., Pilgrim, J., Brooks, T., Goettsch Mittermeier, C., Lamoreux, J. \& da Fonseca, G.A.B. (2004) Hotspots Revisited: Earth's Biologically Richest and Most Endangered Terrestrial Ecoregions. Conservation International \& CEMEX, Washington, D.C., 392 pp.

Mittermeier, R.A., Turner, W.R., Larsen, F.W., Brooks, T.M. \& Gascon, C. (2011) Chapter 1. Global Biodiversity Conservation: the Critical Role of Hotspots. In: Zachos, F.E. \& Habel, J.C. (Eds.), Biodiversity Hotspots. Springer-Verlag, Berlin, Heidelberg, pp. 3-22. https://doi.org/10.1007/978-3-642-20992-5_1

Mertens, J.E.J., Van Roje M., Merckx, J. \& Dekoninck, W. (2017) The use of low cost compact cameras with focus stacking functionality in entomological digitization projects. Zookeys, 712, 141-154. https://doi.org/10.3897/zookeys.712.20505

Moretto, P. (2010) Les Scarabéides coprophages de Bayanga en République Centrafricaine. Bulletin de la Société entomologique de France, 115 (4), 455-477.

Moretto, P., Cosson, B., Takano, H., Basquin, P., Bordat, P., Boucher, S., Bouyer, T., Danflous, S., Dérozier, V., Eitschberger, U., Juhel, P., Leroy, É., Limbourg, P., Massa, B., Meunier, J.-Y., Miles, W., Orbach, E., Robiche, G., Rojkoff, S. \& Silvestre, G. (2021) Un refuge forestier menacé: la forêt d'altitude à Parinari du Mont Tonkoui en Côte d'Ivoire. Évaluation de la biodiversité entomologique. Valeurs de conservation du site. Catharsius La Revue, Hors Série 1, 1-129.

Naskrecki, P. (2008) Sylvan Katydids (Orthoptera: Tettigoniidae: Pseudophyllinae) of the Guinean Forests of West Africa hotspot: an overview and description of new species. Zootaxa, 1712, 1-41. https://doi.org/10.11646/zootaxa.1712.1.1

Naskrecki, P. \& Guta, R. (2019) Katydids (Orthoptera: Tettigoniidae) of Gorongosa National Park and Central Mozambique. Zootaxa, 4682 (1), 1-119. https://doi.org/10.11646/zootaxa.4682.1.1

Olson, D.M., Dinerstein, E., Wykramanayake, E.D., Burgess, N.D., Powell, G.V.N., Underwood, E.C., D’Amico, J.A., Itoua, I., 
Strand, H.E., Morrison, J.C., Loucks, C.J., Allnutt, T.F., Ricketts, T.H., Kura, Y., Lamoreux, J.F., Wettengel, W.W., Hedao, P. \& Kassem, K.R. (2001) Terrestrial Ecoregions of the World: A New Map of Life on Earth. BioScience, 51 (11), $933-938$. [https://academic.oup.com/bioscience/article/51/11/933/227116]

Pimm, S.L., Russell, G.J., Gittleman, J.L. \& Brooks, T.M. (1995) The future of biodiversity. Science, 269, 347-350. https://doi.org/10.1126/science.269.5222.347

Ragge, D.R. (1969) A revision of the African species of Pseudorhynchus Serville (Orthoptera: Tettigoniidae). Bulletin of the British Museum (Natural History) Entomology, 23 (5), 169-190. https://doi.org/10.5962/bhl.part.15133

Ragge, D.R. (1980) A review of the African Phaneropterinae with open tympana (Orthoptera: Tettigoniidae). Bulletin British Museum (Natural History), Entomology, 40, 1-192. [http://www.archive.org/details/bulletinofbritis40entolond]

Ragge, D.R. (1981) A new leaf-resembling genus of bush-cricket from West Africa (Orthoptera: Tettigoniidae). Journal Natural History, 15, 327-330.

https://doi.org/10.1080/00222938100770241

Reid, W.V. \& Miller, K.R. (1989) Keeping options alive. The Scientific basis for conserving biodiversity. World Resources Institute, Washington, D.C., 111 pp.

Sánchez-Bayo, F. \& Wyckhuys, K.A.G. (2019) Worldwide decline of the entomofauna: A review of its drivers. Biological Conservation, 232, 8-27. https://doi.org/10.1016/j.biocon.2019.01.020

Titley, M.A., Snaddon, J.L. \& Turner, E.C. (2017) Scientific research on animal biodiversity is systematically biased towards vertebrates and temperate regions. PLoS ONE, 12 (12), e0189577. https://doi.org/10.1371/journal.pone.0189577

Turner, W.R., Bradley, B.A., Estes, L.D., Hole, D.G., Oppenheimer, L. \& Wilcove, D.S. (2010) Climate change: helping nature survive the human response. Conservation Letters, 3, 304-312. https://doi.org/10.1111/j.1755-263X.2010.00128.x

White, F. (1986) La végétation de l'Afrique. Mémoire accompagnant la carte de végétation de l'Afrique. In: Recherches sur les ressources naturelles. Vol. 20. Orstom \& Unesco, Paris, pp. 1-384, 4 cartes. 